\title{
WestVirginiaUniversity
}

THE RESEARCH REPOSITORY @ WVU

Graduate Theses, Dissertations, and Problem Reports

2005

\section{Prosody in text-to-speech synthesis using fuzzy logic}

Jonathan Brent Williams

West Virginia University

Follow this and additional works at: https://researchrepository.wvu.edu/etd

\section{Recommended Citation}

Williams, Jonathan Brent, "Prosody in text-to-speech synthesis using fuzzy logic" (2005). Graduate Theses, Dissertations, and Problem Reports. 1690.

https://researchrepository.wvu.edu/etd/1690

This Thesis is protected by copyright and/or related rights. It has been brought to you by the The Research Repository @ WVU with permission from the rights-holder(s). You are free to use this Thesis in any way that is permitted by the copyright and related rights legislation that applies to your use. For other uses you must obtain permission from the rights-holder(s) directly, unless additional rights are indicated by a Creative Commons license in the record and/ or on the work itself. This Thesis has been accepted for inclusion in WVU Graduate Theses, Dissertations, and Problem Reports collection by an authorized administrator of The Research Repository @ WVU. For more information, please contact researchrepository@mail.wvu.edu. 


\title{
Prosody in Text-to-Speech Synthesis Using Fuzzy Logic
}

\author{
by
}

Jonathan Brent Williams

Thesis submitted to the College of Engineering and Mineral Resources at West Virginia University

in partial fulfillment of the requirements

for the degree of

Master of Science

in

Electrical Engineering

Approved by

Dr. Norman Lass, Ph.D

Dr. Roy S. Nutter, Ph.D

Dr. Powsiri Klinkhachorn, Ph.D., Chair

Lane Department of Computer Science and Electrical Engineering Morgantown, West Virginia 2005

Keywords: Text-to-Speech, Fuzzy Logic, Intonation, Prosody, Neural Networks

Copyright 2005 Jonathan Brent Williams 


\section{Abstract \\ Prosody in Text-to-Speech Synthesis Using Fuzzy Logic \\ by}

\section{Jonathan Brent Williams}

For over a thousand years: inventors, scientists and researchers have tried to reproduce human speech. Today, the quality of synthesized speech is not equivalent to the quality of real speech. Most research on speech synthesis focuses on improving the quality of the speech produced by Text-to-Speech (TTS) systems. The best TTS systems use unit selection-based concatenation to synthesize speech. However, this method is very timely and the speech database is very large. Diphone concatenated synthesized speech requires less memory, but sounds robotic. This thesis explores the use of fuzzy logic to make diphone concatenated speech sound more natural. A TTS is built using both neural networks and fuzzy logic. Text is converted into phonemes using neural networks. Fuzzy logic is used to control the fundamental frequency for three types of sentences. In conclusion, the fuzzy system produces f0 contours that make the diphone concatenated speech sound more natural. 


\section{Acknowledgements}

I would like to express my gratitude to Dr. Klinkhachorn for his guidance and invaluable advice throughout my project. I would like to thank him for believing in me and for willingly being the chair of my committee. And I would also like to thank him for his patience when my initial thesis topic did not come to fruition. I would also like to thank Dr. Norman Lass for being on my committee and for his guidance throughout my project. His book, "Principles of Experimental Phonetics", guided me in the right direction and was an important asset to my research. I would also like to thank Dr. Roy Nutter for being a member of my committee and for providing me with valuable career advice.

This thesis is dedicated to my late grandparents, Mrs. Madeline Pleasant and Mr. Brent Pleasant. Without them and their sacrifices, I would not have this opportunity.

Special thanks to Dr. Peter Simeonov for providing me with a research assistantship at NIOSH that helped me complete my research. 


\section{Table of Contents}

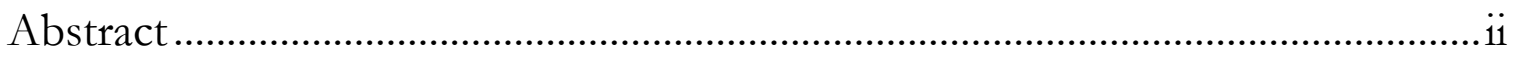

Acknowledgements ...................................................................................................

Table of Contents ................................................................................................ iv

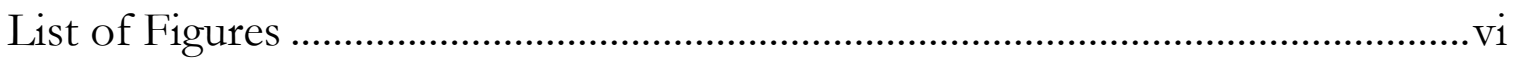

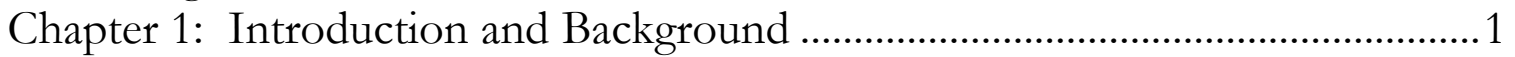

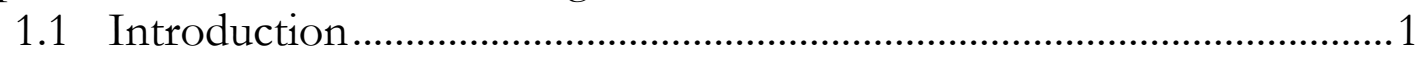

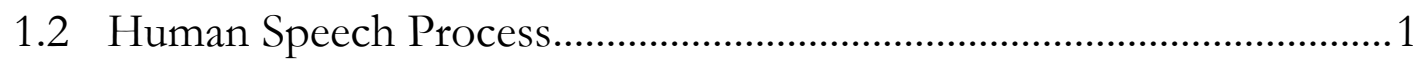

1.3 Speech Synthesis Techniques.....................................................................

1.3.1 Concatenative Speech Synthesis .................................................... 3

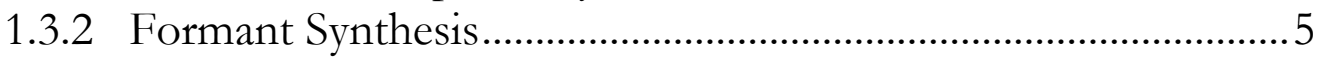

1.3.3 Hidden Markov Model Synthesis .................................................... 5

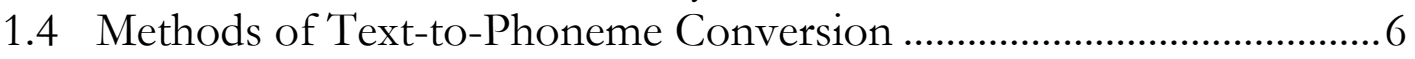

1.4.1 Dictionary-based Approach............................................................6

1.4.2 Rule-based Approach ...................................................................

1.4.3 Machine Learning Approach........................................................

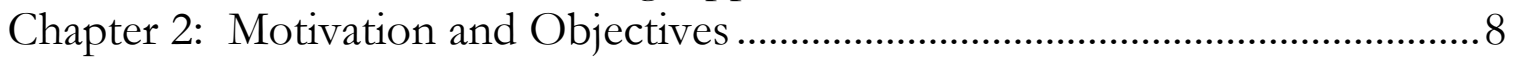

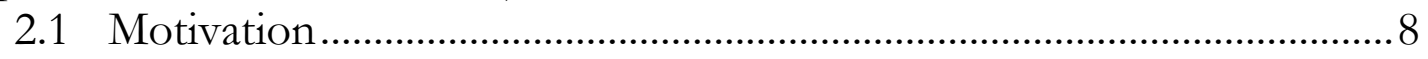

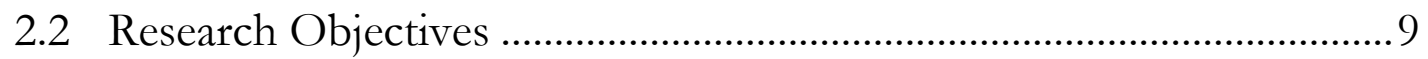

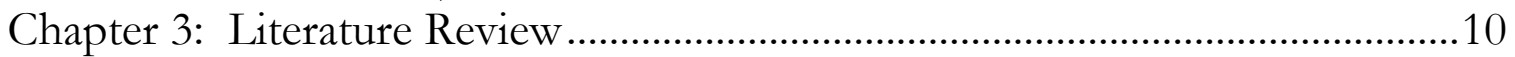

3.1 Generation of Synthetic Speech ........................................................10

3.2 Text-to-Phonemes Algorithms ……………............................................12

3.3 Text-to-Speech Systems ...........................................................................14

3.4 Automatic Prosody Generation...............................................................17

3.5 Back Propagation Algorithm.................................................................21

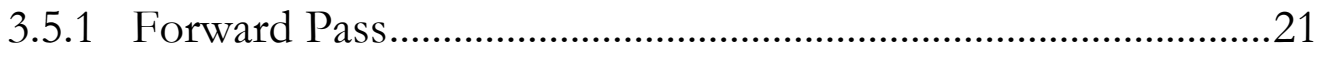

3.5.2 Backward Pass ............................................................................2

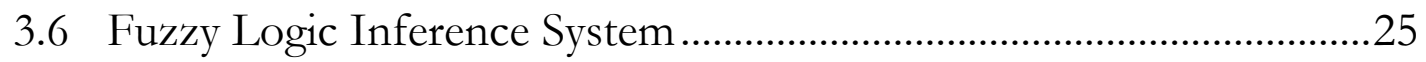

Chapter 4: Text-to-Phoneme Conversion .............................................................28

4.1 Text-to-Phoneme Conversion Overview ……………...........................28

4.2 Text-to-Phoneme Conversion Algorithm...........................................29

4.2.1 Network Input and Output Design ..............................................30

4.2.2 Network Detailed Training Design ..............................................31

4.2.3 Training Set Detail and Alignment................................................35

Chapter 5: Automatic Prosody Generation.............................................................. 37

5.1 Generation of Prosody Overview …………..........................................37

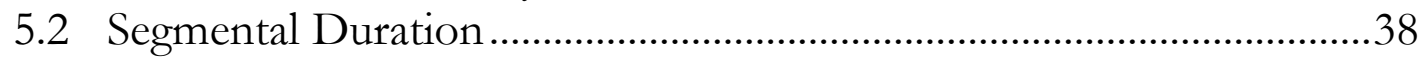

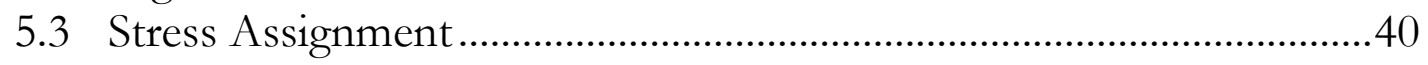




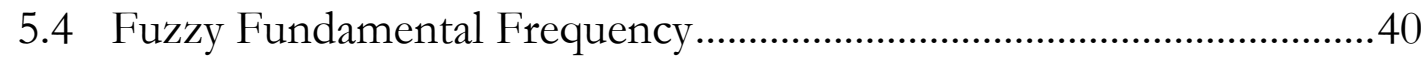

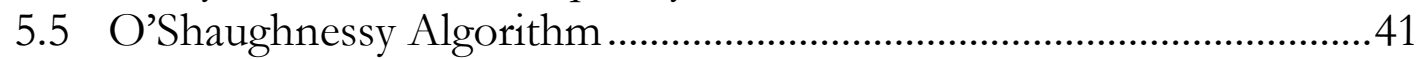

5.6 Fuzzy System Inputs and Consequence ………………….....................4

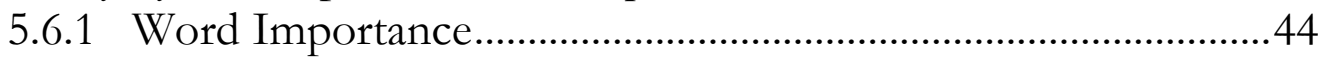

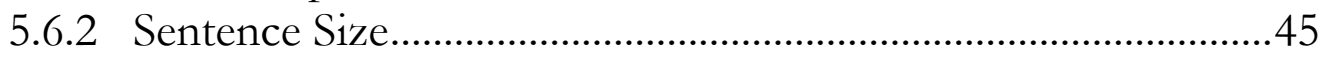

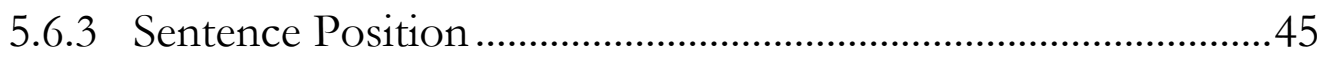

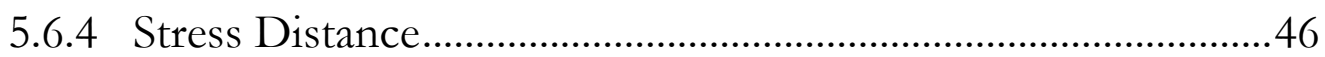

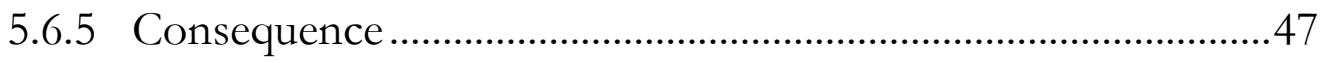

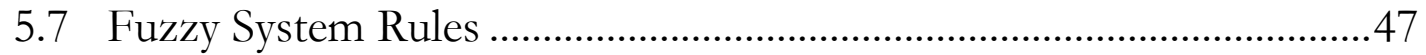

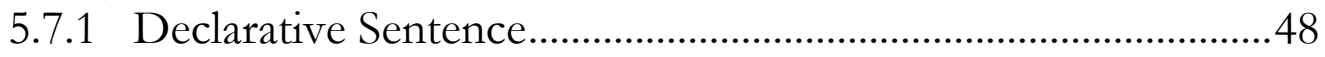

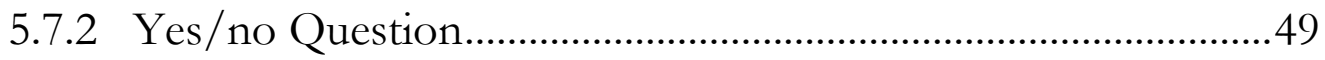

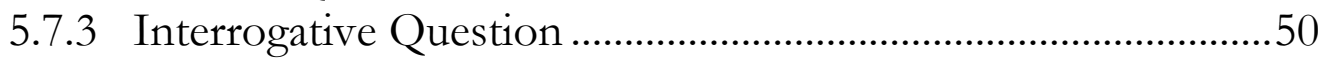

5.8 Fuzzy Output Calculation........................................................................

5.9 Final Speech Production...........................................................................54

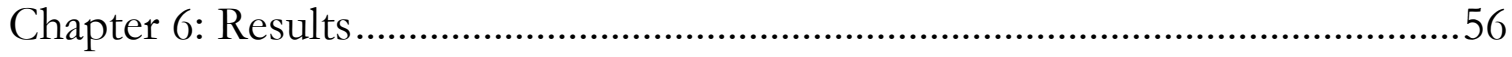

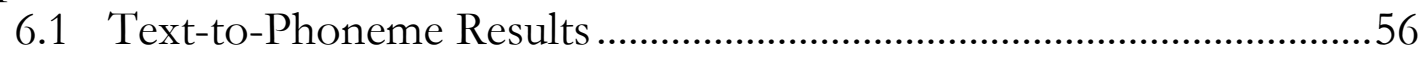

6.2 Fuzzy Fundamental Frequency Results..................................................58

Chapter 7: Conclusion .........................................................................................64

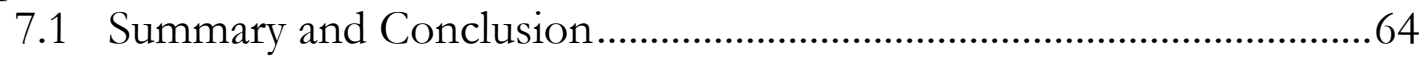

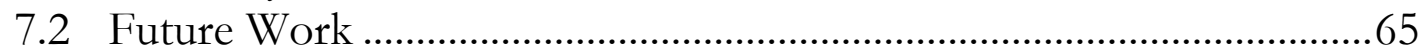

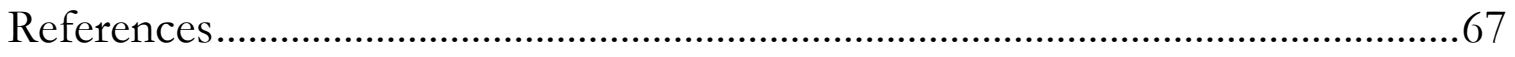

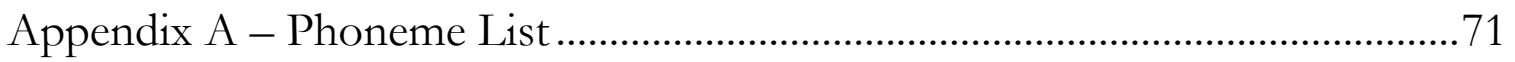

Appendix B - Phoneme Inherit Duration.........................................................

Appendix C - Training Set .................................................................................73

Appendix D - MBROLA Program Description ......................................................85

Appendix E - Source Code ...................................................................................86 


\section{List of Figures}

Figure 1-1: Human Speech Organs............................................................................ 2

Figure 3-1: Wolfgang Von Kempelen's Talking Machine............................................. 10

Figure 3-2: Woman Operating the VODER ............................................................. 11

Figure 3-3: NetTalk Multi-layered Perceptron ............................................................... 13

Figure 3-4: Stevie Wonder Introducing the DECTalk in 1983 ..................................... 16

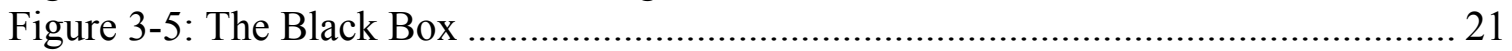

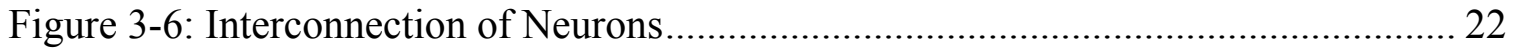

Figure 3-7: The Sigmod Activation Function Neuron.................................................... 22

Figure 3-8: K layered Back Propagation Network ………………………………….... 23

Figure 3-9: Different Types of Membership Functions ..................................................... 26

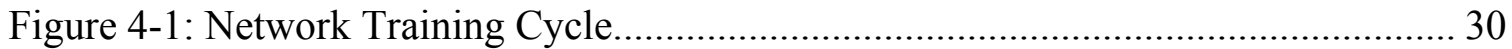

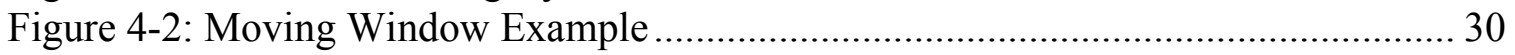

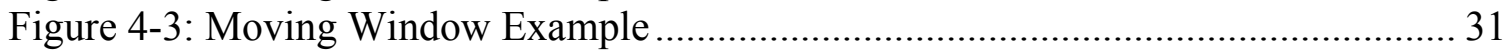

Figure 4-4: Network Training Flow Chart............................................................... 32

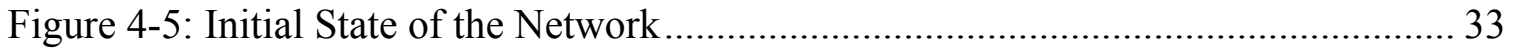

Figure 4-6: State of the Network after First Iteration ..................................................... 33

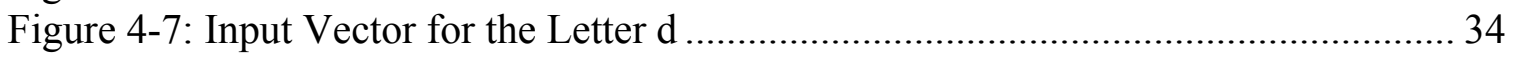

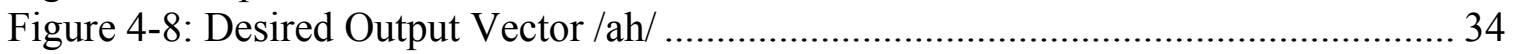

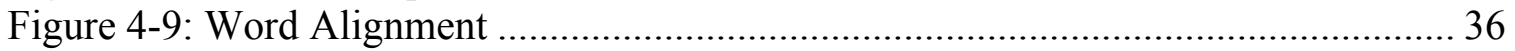

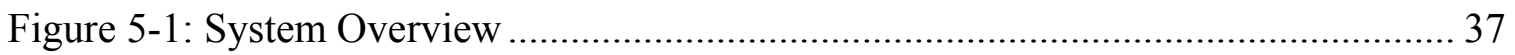

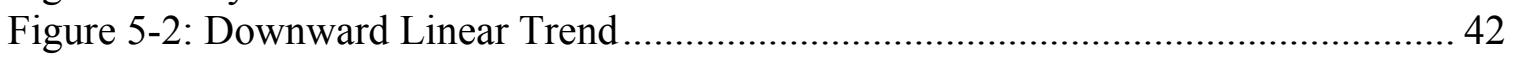

Figure 5-3: Word Importance Input Membership Functions .......................................... 44

Figure 5-4: Sentence Size Input Membership Functions................................................. 45

Figure 5-5: Sentence Position Input Membership Functions............................................ 46

Figure 5-6: Stress Distance Input Membership Functions............................................. 47

Figure 5-7: Consequence Membership Functions ........................................................... 47

Figure 5-8: Example of Word Importance with Calculated Input of 6........................... 52

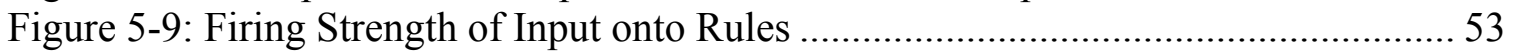

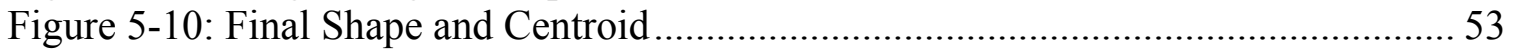

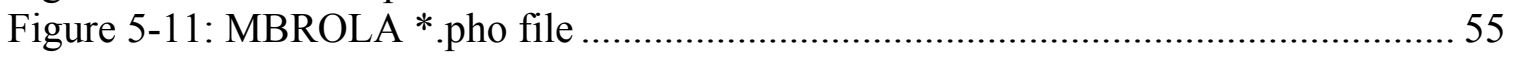

Figure 6-1: Network Convergence............................................................................. 57

Figure 6-2: Declarative Sentence - "My name is Jonathan Williams" ............................ 59

Figure 6-3: Interrogative Question - "What time is the thesis defense?" .......................... 60

Figure 6-4: Yes/no Question - "Is it going to rain at noon?" ............................................ 60

Figure 6-5: F0 Contours for Declarative Sentence, "My name is Jonathan.".................... 61

Figure 6-6: F0 Contours for Interrogative Question, "What time is it?" .......................... 62

Figure 6-7: F0 Contour Comparison for Yes/no Question, “Is it raining today?"............. 63 


\section{Chapter 1: Introduction and Background}

\subsection{Introduction}

Text-to-Speech (TTS) is the process of converting unknown text into sounds that represent the text. Reading out loud is an example of TTS. A TTS system involves converting random text into intelligible synthesized speech. The text can be either directly introduced to the system by a user or scanned from a source [16]. The applications of TTS systems are numerous, from assisting people with disabilities to improving customer service. The ideal TTS system would sound similar to HAL-9000 from the movie, "2001: A Space Odyssey". However, current TTS systems still sound robotic and thus have yet to gain public acceptance. Today, the main problem facing TTS systems is refining the naturalness of synthesized speech. Naturalness of speech is correlated to the prosody of speech. Prosody refers to the intonation, timing, and vocal stress of speech. Currently, TTS research focuses on the improvement of synthesized speech prosody.

\subsection{Human Speech Process}

Synthesizing human speech is difficult due to the complexity of human speech. The production of human speech involves the lungs, the vocal folds, and the vocal tract (oral cavity, nasal cavity, and pharyngeal cavity) functioning collectively. Figure 1-1 shows the organs used in speech production. 


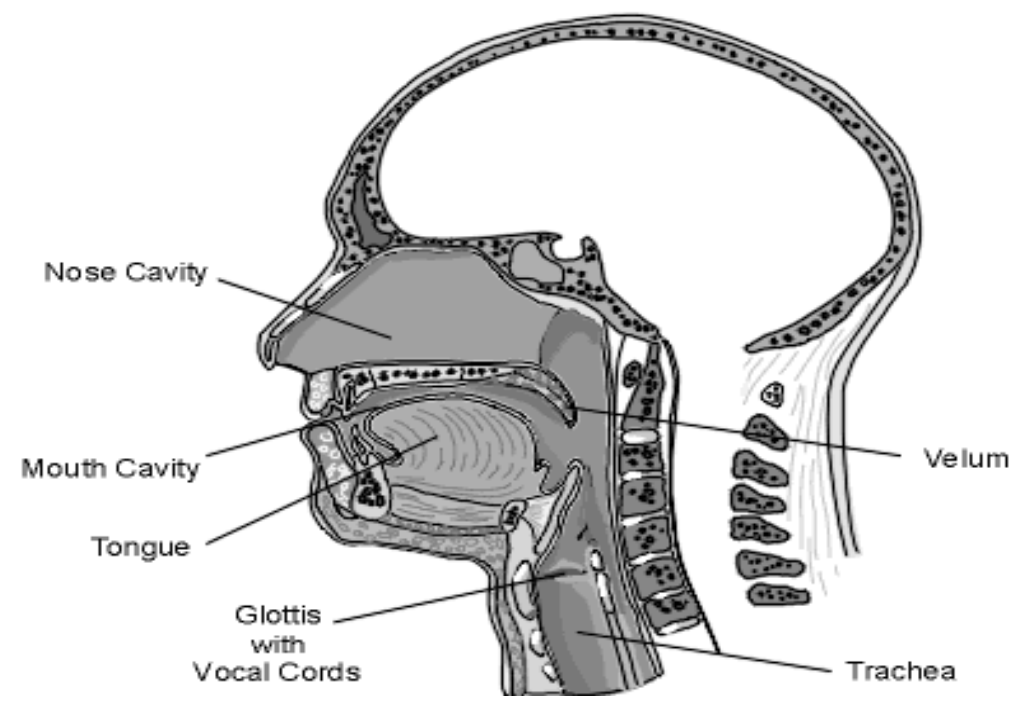

Figure 1-1: Human Speech Organs [40]

Human speech is created by an air source (lungs and the surrounding muscles) causing some type of excitation in the vocal system (vocal folds and vocal tract). The type of sound produced is determined by the vocal system's affect on the air flow. There are two types of speech produced by humans; voiced and unvoiced. With voiced speech, sound is produced from the vibration caused by air flowing through tensed vocal folds. Unvoiced speech is created from air flowing through abducted vocal folds, and the sound is produced by air flowing through a constriction in the vocal tract or air being stopped and then suddenly released [40].

Mimicking the sounds created by human speech is difficult because real continuous speech is a combination of many complex audio signals. With voiced speech, the speech signal is modified by either the oral cavity or the nasal cavity. These cavities act as resonators with pole and zero frequencies. Pole and zero frequencies are called formant and anti-formant frequencies, respectively. These frequencies have their own amplitude and bandwidth. Voiced speech also produces a complex quasi-periodic pressure wave from an interruption in air flow caused by the vibration of the vocal folds. The frequency of impulses from the pressure wave is called the fundamental frequency. With purely unvoiced speech, since there is no vibration of the vocal folds, there is no fundamental frequency.

Researchers believe that the most important signals generated by human speech are the formant frequencies and the fundamental frequencies. When synthesizing speech, 
these signals greatly contribute to the naturalness of the speech. The formant frequency represents the shape of the sound that is formed by the vocal tract (oral cavity and the nasal cavity). Different sounds (vowels, nasals, etc.) within a language are distinguishable by their formant frequencies. The fundamental frequency determines the pitch of the voice. For example, women and children have a higher pitch (i.e. higher fundamental frequency) than men. Throughout the years, research involving the naturalness of synthesized speech has focused primarily on these two acoustic features.

Sounds created by humans are merely noise if the sounds do not have meaning. Sounds in speech production are categorized into units. These units can be as large as words or as minute as a phone. However, phonemes are the fundamental units of phonology. The definition of phonemes is the theoretical unit of sound that can distinguish words [29]. The concatenation of phonemes produces the words in the language, i.e. changing a phoneme means changing the word. Phonemes are split into two major categories: vowels and consonants. All vowels are voiced sounds while some consonants are voiced sounds and some are unvoiced sounds. The number of phonemes on a language depends of the actual language, speaker, and the particular dialect. For example, most standard American English consists of 41 phonemes. Diphones are the stable middle region between two phonemes. Diphones represent the transition between two phones. Therefore, the number of diphones in a language is the number of phonemes squared. With speech synthesis, the role of phonemes and diphones is to focus on sounds that the system should yield.

\subsection{Speech Synthesis Techniques}

Speech synthesis is the artificial production of human speech. The techniques of speech synthesis are categorized into three different approaches. These are concatenative speech synthesis, formant-based synthesis, and Hidden Markov Model synthesis.

\subsubsection{Concatenative Speech Synthesis}

Concatenative speech synthesis involves combining previously recorded speech to form words or sentences. The concatenative speech synthesis approach currently 
produces the most natural sounding speech because of the use of real speech. There are three methods of concatenative speech synthesis: diphone synthesis, domain-specific synthesis, and unit-selection synthesis. The most commonly used methods today are domain-specific and unit-selection synthesis.

Diphone synthesis involves the concatenation of diphones. As previously explained, diphones are the middle region between two phones. The concatenation of diphones minimizes the co-articulatory effects of the phone to phone transition. During synthesis, digital signal processing (DSP) techniques such as linear predictive coding (LPC), POLA, and MBROLA are used to overlay the desired prosody onto these diphones. The amount of diphones for a given language is equal to the number of phonemes of that particular language squared. Therefore, the diphone inventory size is relatively small and diphone synthesis can be used with inexpensive processors and embedded systems. However, diphone concatenation yields robotic sounding speech due to the digital signal processing.

Domain-specific concatenation is a very common form of speech synthesis. Most companies use domain-specific synthesis for their phone-based customer service systems. Consequently, this type of synthesis has gained some notoriety over the years. Domain-specific synthesis concatenates pre-recorded words and phrases to create speech. Domain-specific synthesis is only useful in applications where the output is restricted to a certain domain. Examples of domain-specific synthesis are automatic reports of the weather, talking gadgets, automated banking, and automated customer service. Domain-specific concatenation sounds natural because the variety of sentence type is limited; therefore, the output matches the original recording. However, since this method of synthesis is limited to only specific applications, domain-specific synthesis could not be implemented in a TTS system.

Unit selection-based synthesis is the method currently used in commercial-based TTS systems. Unit selection is created from large databases of hours of recorded speech. These recordings are then converted into units to be concatenated into speech. First, a recording of speech is segmented into individual phonemes, syllables, words, phrases, and sentences. The segmentation is done by hand or automatically by a modified speech recognizer. An index of the units from the speech database is then created based upon 
prosodic parameters, like fundamental frequency and segment duration. Synthesis is created using a decision tree that determines the best path of candidate units from the database. Unit selection yeilds the most natural sounding speech today. However, to achieve maximum naturalness requires unit selection-based systems' speech databases to be gigabytes in size [36].

\subsubsection{Formant Synthesis}

Formant synthesis generates synthetic speech using formants. Formants represent the resonant frequencies of the oral cavity or the nasal cavity. An acoustic model is used to create the speech and parameters, like fundamental frequency, spectral components, and noise levels which are varied over time to create a waveform of speech. The disadvantage of formant synthesis is the robotic sounding quality of speech [36]. However, formant synthesis does have many advantages over concatenative speech.

Formant synthesis does not require stored databases and thus requires little memory. Formant synthesis can also be generated at very high speeds. This makes formant-based systems useful in embedded computing and real-time applications. For example, most reading machines for the blind use formant-based synthesis. In addition, formant-based systems offer the user more control over the output speech. With formant synthesis, intonation and prosody can be altered to represent an assortment of emotions and tones of voice.

\subsubsection{Hidden Markov Model Synthesis}

Hidden Markov Model (HMM) synthesis is the process of modeling the speech output using the Markov process. The HMM is a Markov process where the parameters are unknown. With HMM, each state has outputs, and future states are dependent only on the present state [36]. HMMs are used in speech synthesis to model the vocal tract and prosody. The quality of HMM speech synthesis is good but not as good as unit selectionbased systems [36]. HMM synthesis is a newer method of speech synthesis and has been applied mostly to trainable TTS systems. 


\subsection{Methods of Text-to-Phoneme Conversion}

In order for a TTS system to be accepted by the public, the system must correctly convert the inputted text into the correct pronunciation. Text-to-Phoneme (TTP) conversion consists of translating text into its phonetic transcript. This task can be accomplished using many different methods. The most common methods are the dictionary-based approach, the rule-based approach, and the machine learning approach.

\subsubsection{Dictionary-based Approach}

The dictionary-based approach is the easiest TTP conversion method to implement. Dictionary-based TTP conversion consists of storing phonological knowledge into a lexicon [16]. Early dictionary-based conversions required locating word pronunciations that were stored in a large lexicon. This process required enormous amounts of memory because the lexicons had to contain every word and its pronunciation. Most dictionary-based TTP conversions today use stored morphemes instead of words in the lexicon. Morphemes are the smallest language unit that carries a semantic interpretation. For example, the word "uncontrollable" contains three morphemes; "un-", "-control-“, and "-able”. Morphemes require less memory to store and can cover most words in a language. Both types of dictionary-based conversion methods handle unknown words similarly. Rules are used to pronounce unknown words or morphemes [16]. The main drawback of dictionary-based TTP conversion is the amount of memory that the lexicon can consume. Yet, dictionary-based systems are the easiest to create.

\subsubsection{Rule-based Approach}

The rule-based TTP conversion approach uses expert rules to yield pronunciations. The pronunciations are based on the spelling of the word. These rules are similar to the sounding-out rules used by grade school students when learning how to read [36]. There are some drawbacks to the rule-based approach. For instance, rule-based TTP conversion system rules can become very complex, especially with irregular languages like the English language. Rules are much more complex to code than simple 
binary searches used by dictionary-based TTP systems. However, rules-based systems can work on any input presented to the system.

\subsubsection{Machine Learning Approach}

There are many different types of machine-learning approaches to TTP conversion. Machine learning is the ability of a machine, a computer, or an electronic device to improve its performance based upon previous results. Machine learning requires that a system "learns" how to convert text into its phonetic representation. With TTP conversion, this task can be accomplished with many different methods. Neural networks, Self-Organizing Maps, and Decisions Trees are examples of the machine learning approach. However, for irregular languages like English, the accuracy is not equivalent to the latter approaches. 


\section{Chapter 2: Motivation and Objectives}

\subsection{Motivation}

TTS systems are becoming more commercially available as the quality of the systems improves. Most commercial systems use the unit-selection based concatenation approach to produce speech output [36]. However, unit-selection requires large prerecorded speech databases that must be segmented in order to create a useable system [36]. Segmentation of a prerecorded speech database can be very difficult and timely. Formant synthesis uses less amounts of memory, but the speech is very mechanical in sound. For speech synthesis to be widely accepted in robotics and our shrinking electronics, speech synthesis systems must use as little memory as possible, must require little effort to create, and must sound somewhat natural.

Diphone concatenation is adequate enough to produce understandable synthesized speech. Most languages consist of about 2000 diphones. Diphone inventories take relatively little memory to store, and there are many freely available programs that use diphone concatenation. However, diphone concatenation systems produce speech that is robotic and not the quality of current unit-selection based systems. Prosody is what makes speech sound natural, and the prosody in recorded speech segments is more natural sounding than that of synthesized prosody.

This thesis presents a different method to produce more natural sounding speech for diphone concatenation-based TTS systems. Most TTS systems that use diphone concatenation use either neural networks or rule-based approaches to generate prosody. This research exams the use of Fuzzy Logic to generate one aspect of prosody: fundamental frequency. TTP conversion is performed using the neural network Back Propagation algorithm. Using linguistic data as input, the fuzzy logic system produces fundamental frequency and frequency contour as outputs. The rules of the fuzzy logic system are from the O'Shaughnessy fundamental frequency algorithm used in the MITalk system [2]. As a result, the TTS system output speech should sound more natural. 


\subsection{Research Objectives}

The research objective is to apply the flexibility of fuzzy logic to the randomness of TTS conversion in order to produce more natural sounding synthesized speech. Using established information provided by the user of the TTS system, the fuzzy controller will produce output that will reflect the user's input.

The specific research objectives are:

1. Gain an understanding of the process of human speech production.

Understand the concepts and theories of generating synthetic speech. Research different algorithms that have been created to generate English prosody automatically.

2. Build an English text-to-phoneme system using neural networks that will produce accurate phonemes given inputted text. The Back Propagation algorithm will be used to train the network. The training set will consist of about 1800 words.

3. Implement a fuzzy control system that will control the intonation of the speech. The fuzzy controller will receive different linguistic parameters as the input and produce fundamental frequency as the output. This fuzzy controller will represent the O'Shaughnessy fundamental frequency algorithm and system will handle three types of sentences: declarative, yes/no question, and interrogative questions. The final system should produce understandable speech that mimics the proper intonation for each type of sentence.

4. Calculate the accuracy of the text-to-phoneme network using the training set and unknown text. Evaluate the fuzzy controller by comparing the f0 contour produced by the controller with the f0 contour of the Microsoft Research Speech Technology Asia (MRSA) on-line TTS system [37]. 


\section{Chapter 3: Literature Review}

TTS research has been extensively examined over the last 40 years. This chapter presents an extensive literature review of past and present research on the different aspects of the TTS system. The review has been performed on the following subjects: generation of synthetic speech, TTS conversion algorithms, TTS systems, automatic prosody generation, and the fuzzy logic inference system.

\subsection{Generation of Synthetic Speech}

Generating synthetic speech has been a curiosity for the past 1100 years. Around the year 1003, Gerbert of Aurillac created the first known mechanical talking machine. For the next two centuries, inventors like Albertus Magnus and Roger Bacon created machines know as "talking heads" [23]. However, the first known machine that tried to mimic real human speech was developed by Christian Kratzenstein of St. Petersburg in 1779. This machine could produce five long vowel sounds. Twelve years later, Wolfgang Von Kempelen developed a machine that could produce vowels and some consonants $[21,33]$.

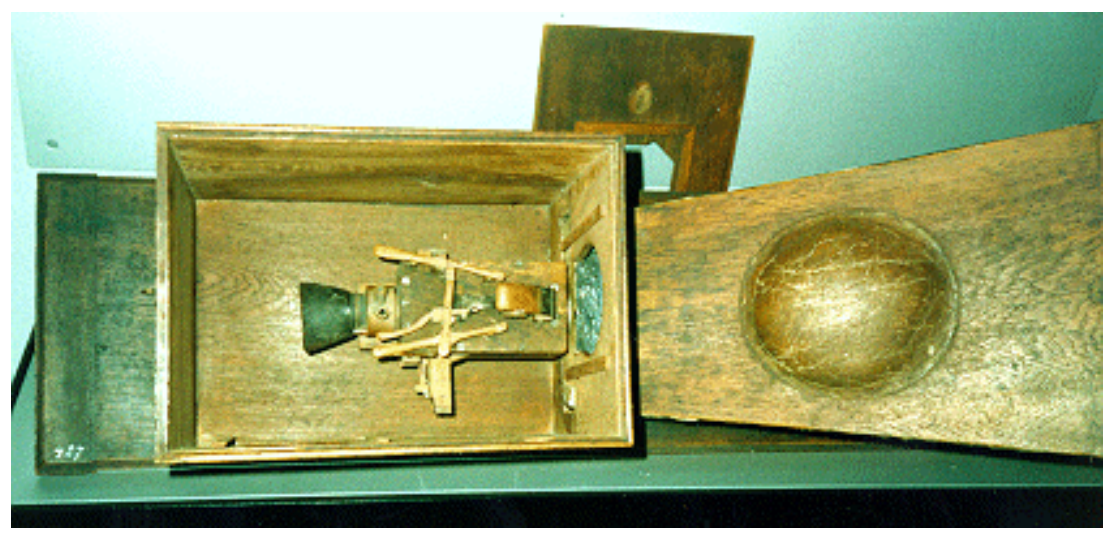

Figure 3-1: Wolfgang Von Kempelen's Talking Machine [33]

With the start of the $20^{\text {th }}$ century and the increasing use of electricity, speech synthesis began to move from mechanical machines to electrical machines. 
Electronic speech synthesizers were first developed in the 1920's. The first known electronic synthesizer, VODER, was developed by Homer Dudley in the late 1930's [21]. Dudley was a research physicist at the Bell Laboratories in New Jersey. Dudley reconstructed the Bell Laboratories speech analysis, VOCODER, into the speech synthesizer VODER. The VODER was controlled by an operator using a keyboard to

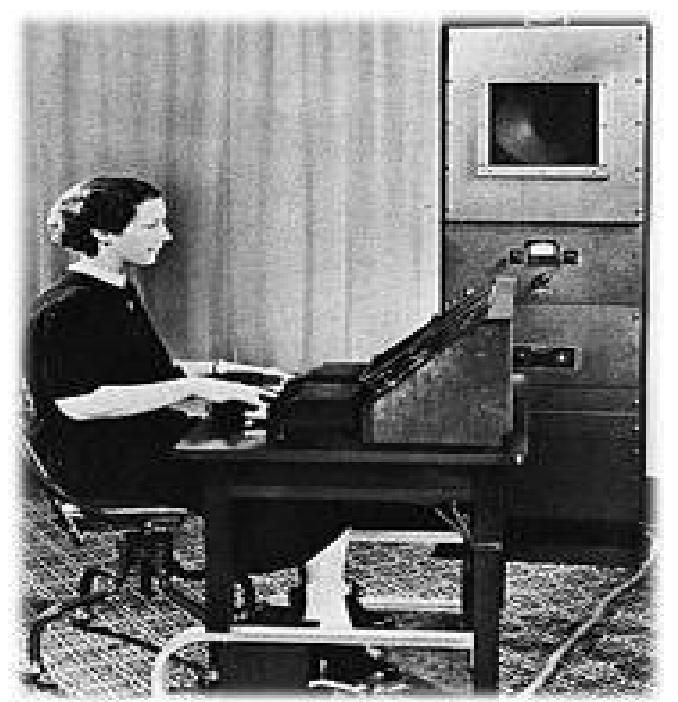

Figure 3-2: Woman Operating the VODER [36]

adjust the filter output, foot pedals to control the fundamental frequency, and special keys to create closure and the release required for stops [23]. The VODER was operated like a musical instrument. Eventually, human operated machines became obsolete. After World War II, the spectrograph provided a new tool for researching acoustic phonetics. As a result, researchers began to study speech based on acoustical data.

In the 1950's, speech synthesizers like the Pattern Playback were developed to produce speech from copied speech waveforms. The speech synthesis by rule approach (formant synthesis) began to become prevalent in the following decade. Concatenative speech synthesis became a focus of research in the 1970's with the initial focus on phoneme concatenation. However, it was quickly discovered that diphone concatenation would be more feasible than phoneme concatenation [21]. In 1976, Olive and Spickenagle used linear prediction speech analysis to automatically create a full diphone inventory for concatenation [27]. In 1988, Nakajima and Hamada wrote about a method of speech concatenation that used a unit-selection based approach, instead of the more 
common diphone concatenation approach [25]. Today, diphone concatenation and unitselection concatenation are the most common methods of speech synthesis with the latter becoming more common commercially.

\subsection{Text-to-Phonemes Algorithms}

Today, there are many different ways to produce speech from text. One of the earliest methods of converting text to phonetics was the use of sophisticated heuristics [21]. The first full English TTS system used this method in combination with a syntactic analysis module. As computer memory increased, the preferred method for TTP conversion was the use of a look-up dictionary. Look-up dictionary algorithms consist of matching inputted words to words in a lexicon, utilizing phonological rules as a back-up. By the 1980's, TTS systems like the KlatTalk and DECTalk used a combination of a look-up dictionary and phonological rules [2, 21,22]. As computer technology became

more sophisticated and accessible, researchers began to develop new ways of tackling the TTP conversion problem.

The use of machine learning techniques for TTP conversion was researched in the 1980s. In 1987, Sejonowski and Rosenberg used neural networks to convert inputted text into phonemes. This system used a 120 hidden neuron multi-layered perceptron trained with 2000 words [35]. Neural networks use neurons with weights that represent the neurons and the synapse of the neurons, respectively. Each weight represents the firing strength of the neuron synapse. Initially, all of the weights for each neuron in the network are randomized. The weights are updated using the calculated error from the training data and the network's actual output. In the multi-layered perceptron algorithm, the error is propagation backwards throughout the layers of network. Figure 3-3 shows an overview of the NetTalk network. 


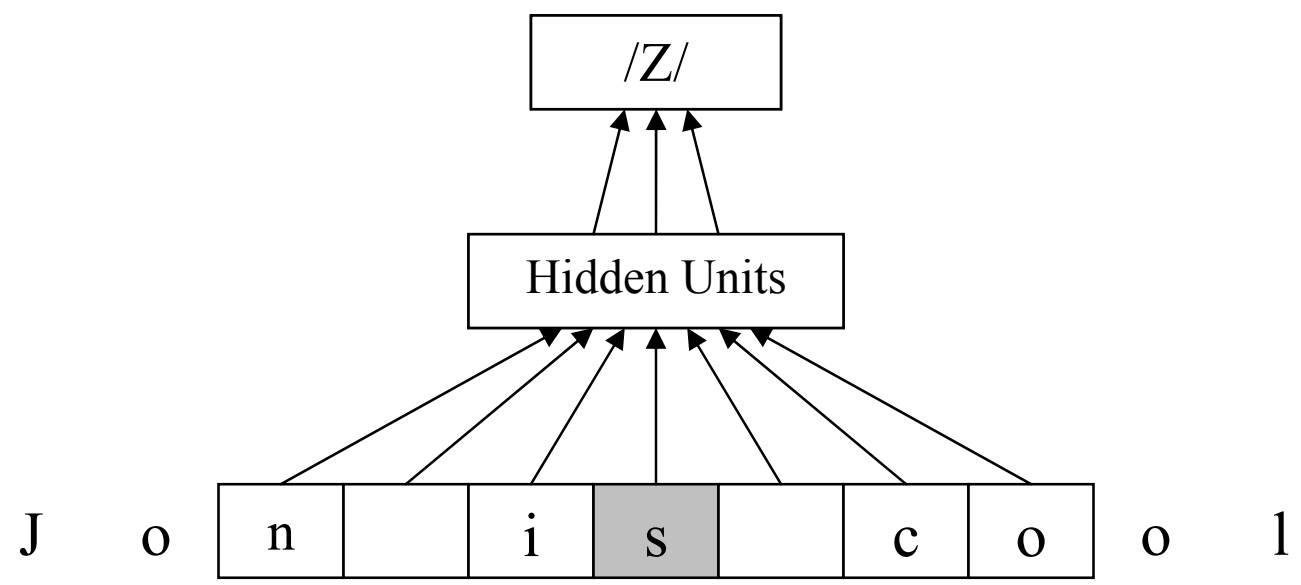

Figure 3-3: NetTalk Multi-layered Perceptron

McCulloch devised a re-implementation of the NETTalk multi-layer perceptron algorithm called NETSpeak, which yielded about 85\% accuracy [24]. Recently, Arciniegas and Embrechts used a staged neural network algorithm to handle some of the previous problems of the single-staged multi-layer perceptron algorithm. In that paper, two stages of neural networks were used to convert text to phonemes. One stage separated regular words from special words. Special words were categorized as words that contained single letters represented by two output phonemes. The other stage found the phonetic output for the two types of words [4]. Gubbins used a hybrid-neural network approach which used both neural networks and a simple rule-based system to convert text to phonemes [18]. The multi-layer perceptron neural network is not the only neural network algorithm researchers have used for TTP conversion.

Adamson and Damper researched different ways to improve the performance of TTP conversion neural networks [24]. They used a recurrent neural network which addressed some of the problems of the multi-layer perceptron algorithm. A recurrent neural network uses a single letter as input and trains itself using the Back Propagation through Time algorithm. This algorithm removes the need for alignment of the training set. The network has a recurrent structure with no constraints regarding which direction units can interact [19]. Adamson and Damper's algorithm initially performed worse than the NETTalk and NETSpeak, but their paper was the foundation for other recurrent neural network based algorithms [1]. In 2004, Bilcu, Astyola and Saarinen improved the performance of the recurrent neural network algorithm by using three letters as the input 
to a recurrent neural network [6]. In 2003, Bilcu compared the performance of multilayered perceptron to two types of recurrent networks. The results showed that the multilayered perceptron is the most accurate of the three [7]. Besides neural networks, TTS systems have also used other forms of machine learning to convert text to phonemes.

The pronunciation by analogy (PBA) algorithm is another machine learning algorithm that has been used for TTP conversion. PBA is similar to the dictionary-based algorithms because both algorithms use dictionaries to convert text to phonemes. However, the PBA algorithm uses a different method to handle words not in the dictionary. The PBA algorithm concatenates partial pronunciations of substrings using learned phonological knowledge [12]. Using an aligned lexicon, pronunciation can be achieved through explicit and implicit learning. An example of a system that uses this algorithm is the PRONOUNCE system [12, 13]. In the PRONOUNCE system, an inputted word is matched to words in a dictionary. Then substrings with common letters are found between the found dictionary entry and the input. Phonetic substrings are also built from the matched substrings. Information from these substrings is then used to build a pronunciation lattice. Lattice nodes are first labeled with $\mathrm{L}_{\mathrm{i}}$ and $\mathrm{P}_{\mathrm{i}}$ representing the matched letter and the corresponding phoneme in the substring, respectively. $\mathrm{P}_{\mathrm{i}}$ is labeled $P_{i m}$ to represent the $m_{t h}$ matched substring. If there is a match between two $L_{i}$ and $L_{j}$, then an arch is placed between the two nodes with the $\mathrm{P}_{\mathrm{im}}$ and $\mathrm{P}_{\mathrm{jm}}$ being the arc labels. The pronunciation for an unknown string is created by the best path through the lattices [12]. PBA do suffer from one major drawback however. Due to incomplete paths, PBA tend to have silences during text conversion. This problem was solved in Sullivan and Damper, but the pronunciation lattices' size greatly increased [39].

\subsection{Text-to-Speech Systems}

The first full TTS system was developed in the late 1960's. Since then, there have been many advances in the accuracy and the quality of TTS systems. Companies like IBM, Microsoft, and Bell Labs have developed both free and commercially available systems. 
In 1968, Umeda of Japan developed the first demonstrated TTS system for the English language. This system transformed text to phonemes using linguistic rules. Sentence pauses were placed in sentences with ten or more syllables [21]. In 1973, the Haskins TTS system was developed but was later discontinued due to inadequate research funds. This system used the Kenyon and Knott 140,000 word phonetic dictionary with a rule system to handle unknown words. The Haskins TTS system was developed to aid the blind with reading. Although the system was never produced commercially, the Haskins TTS system is considered a significant step in TTS research [21].

In 1976, Allen, Hunnicutt, and Klatt developed the MITalk at MIT [2]. This TTS used different levels to convert text to synthesized speech. In the first level, abbreviations, numbers, and symbols were transformed into words. Then, using a 12,000 morph (prefixes, roots, and suffixes) lexicon, words were converted to their phonetic equivalent. Words not in the lexicon were converted to phonemes by using rules. Stress and "part of speech" for each word was determined on another level. Then the final level produced the synthesized speech. Phoneme, syllabic, and pause duration were determined using the Klatt duration rules [20,2]. Fundamental frequency contour was determined using an adaptation of the O'Shaughnessy algorithm [28, 2]. The f0 contour was smoothed, and the waveform was generated using a terminal synthesizer.

The Klatttalk TTS system was developed in 1983 by Dennis Klatt. Dennis Klatt had previously worked on the MITalk system a few years earlier. This system used the Hunnicutt letter-to-phoneme rule system plus an exception dictionary to convert text to speech. The Klattalk was more rule-based than the dictionary-based MITalk system [22]. The Klattalk system was the basis for the 1982 Digital Equipment Corporation DECTalk. The DECTalk system later became commercially available in 1983. DECTalk was very versatile because of its ranges of voices and different speech speeds. Due to the flexibility of the DECTalk hardware, the DECTalk was easily updated with improved versions of the Klattalk system [21]. 


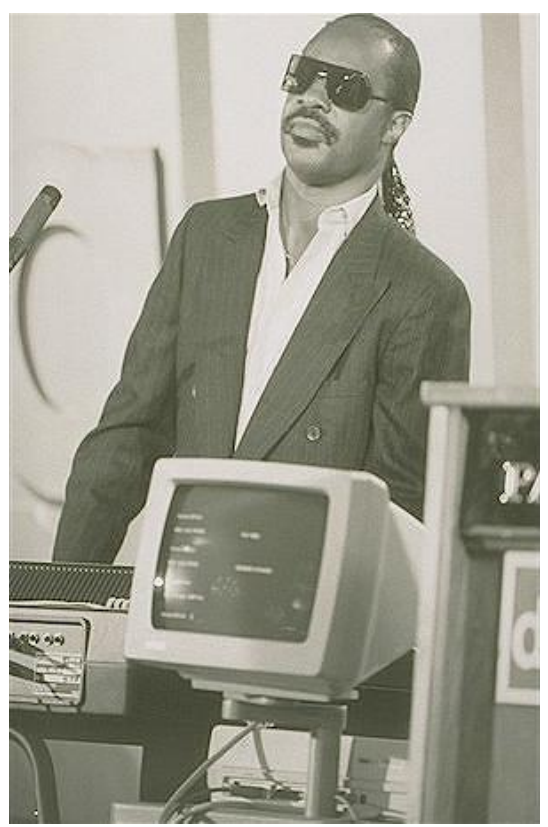

Figure 3-4: Stevie Wonder Introducing the DECTalk in 1983 [41]

In the early 1980's, Richard Gagnon developed a very inexpensive segmental synthesis program. This system became the commercial Votrax Type-n-Talk. The system was built with very inexpensive hardware and a small phoneme inventory to synthesize speech. The Echo TTS system was another inexpensive segment-based system which used a diphone inventory and a linear predictive synthesizer to produce speech $[21]$.

Today, there are many types of TTS systems for many different purposes. The Festival Text-to-Speech system developed by Black and Clark is an example of current TTS systems developed for research. Festival uses letter-to-sound rules and a large lexicon for TTP conversion. Speech synthesis is accomplished using unit-selection concatenation of diphones [8]. The MBROLA project is a TTS system that is freely available for researchers. The MBROLA project was developed by TCTS Lab of the Faculté Polytechnique de Mons in Belgium and is a back-end system [15]. This system is to be used as a speech producer for a TTS system developed by the user. MBROLA uses diphone concatenation to produce speech for many different languages. Microsoft has many English TTS systems for different applications. Microsoft based products use either unit-selection or diphone concatenation to synthesize speech. Microsoft Reader is free software from Microsoft that converts text from e-mails and other documents to 
speech. The Apple PlainText TTS system is standard on PowerPC computers. The PlainText uses a dictionary-based system to convert text to sounds and diphone concatenation to produce speech. A very high quality commercial system is the AT\&T Natural Voices TTS system. The AT\&T Natural Voices enables users to define the pronunciation of certain words. This system uses unit-selection synthesis to produce speech [5].

\subsection{Automatic Prosody Generation}

Currently, prosody is the major issue in speech synthesis. As a result, most research deals with prosody of speech. Prosody, in the context of speech, consists of the properties of speech, such as pitch, loudness, and duration [16]. Prosodic events can be phonemes, syllables, or words. Prosody in a speech system mainly deals with fundamental frequency and segmental duration.

Segmental duration refers to the timing of the units that create speech. These units can be either as small as a phone or as large as a phrase. The size of the units must be determined in order to adequately model real speech. Researchers know that subphonetic segments do exhibit different durations. However, this information would be too complex for TTS systems, so most research is focused on either the phonetic or the supra-phonetic sized segments [16]. Early research on segmental duration addresses the principles of isochrones. A speaker would unconsciously use an internal clock while speaking. As a result, Campbell contended that segments in a syllable frame are found using the following formula:

$$
D u r_{\mathrm{i}}=\exp \left(\mu_{i}+\mathrm{k} \sigma_{i}\right)
$$

$D u r_{\mathrm{i}}$ is the duration of the segment at syllable $\mathrm{i}_{\mathrm{i}}$ and $\mu_{i}$ and $\sigma_{i}$ statistical measures of a large corpus. However, this formula is too strict of an application to accurately model duration [15].

More accurate segmental duration models have focused primarily on the phoneme as the segmental unit. Although other segments are taken into account, these segments 
do not directly affect the duration of the units. Currently, there are two types of segmental duration models: rule-based models and corpus-based models. Rule-based models use rules to modify intrinsic durations, while corpus-based models use sophisticated methods to automatically derive models using data within the corpus [16].

One of the best known rule-based duration models was developed by Dennis Klatt in 1976. This model has been used in MITalk, Klattalk, and DECTalk TTS systems. Each phoneme consists of an inherent duration and a minimum duration [2]. There are eleven rules that alter the duration of the phoneme due to factors like their location, their manner of articulation, their stress, etc. The duration of the phonemes is changed by using the formula:

PRCNT1 $=(P R C N T 1) *(P R C N T 2) / 100$

Where PRCNT1 represents the current duration of the phoneme, and PRCNT2 is a number that the phonemes need to be altered [2]. Although this duration model works well, recent research has focused more on the corpus-based approach to duration modeling.

Corpus-based duration models take advantage of the advancements in computational resources. Using a large recorded speech corpus, parameters are extracted and models are created using some type of abstract learning method. In 1994, Riley used a corpus of 400 utterances from a single speaker and 4000 utterances from 400 speakers to model segmental duration. From this corpus, Riley built a classification and regression tree (CART) as the segmental duration model [32]. In 1992, Campbell used a neural network to model segmental duration. In that paper, he computed syllable duration independent of inherit segmental durations. Campbell believed that previous rule-based models were incorrectly based on inherent durations. His focus was to model higher levels of prosodic structure like syllables and prosodic phrases. Consequently, Campbell's network was trained to model Japanese and English syllables [11].

Although segmental duration is a very important aspect of speech synthesis prosody, it is not as essential as pitch and intonation. For example, a modification in pitch can change a statement into a question. Initially, research on this aspect of prosody 
mainly focused on the relationship between intonation and stress. Intonation deals with the pattern of tones in an utterance. Stress deals with the emphasized syllable of a word. It was believed that there was a direct association between intonation and pitch and that stress was created by changes in vocal intensity and syllable duration [21]. However, it is now known that the change in the fundamental frequency indicates stress and intonation [21]. Over the years, there have been different theories to predict the rise and fall of fundamental frequency (f0).

In the mid 1960's, Mattingly developed a fundamental frequency theory that used three tunes placed on the last prominent syllable of a clause. The tunes were rise, fall, and rise-fall which correspond to a statement end, a question end, and a continuation rise, respectively. Later in the decade, researchers tried to develop models that mimic the exact fundamental frequency contour of natural speech. In 1969, Öhman stated that f0 contours can be modeled in terms of a discrete signal fed to a linear smoothing filter [21, 26]. A Japanese intonation model by Fujisaki was able to closely match natural intonation contours using the ideas proposed by Öhman [17]. Fujisaki listed two types of events: phrase and accent command [16]. Phrase commands were modeled as a pulse function, and accent commands were modeled as a step function [16]. Hart and Cohen described intonation as a hat pattern. The fundamental frequency will rise on the first stressed syllable of a phrase and then remain high until the final stressed syllable. At the end of the phrase, there will be either a large fall or a fall-rise of the fundamental frequency [21].

The O'Shaughnessy fundamental frequency algorithm was developed in 1979. This rule-based method first assigned peaks to stresses in the sentence. The size of the peak depended on the length of the sentence, the location of the stress, and the importance of the word. Then rises and falls were placed around the peaks. The final rise or fall of the sentence depended on whether the sentence was a statement, a yes-no question, or an interrogative question [2]. The Pitch Contour theory was developed in the late 1960's for British English. This theory splits speech into four components: prehead, head, nucleus, and tail [16].

By the early 1980's, models for fundamental frequency generation began to become more comprehensive and flexible. A well known model was developed in 1984 
by Anderson and Pierrehumbert [30]. Anderson and Pierrehumbert believed that stress patterns in a sentence affected the fundamental frequency contour. This model advances on some of the earlier research that used two tones. The model separates intonation into two main tones, a high and a low [30]. These tones are placed on stressed syllables as a single tone or a combination of tones. The sequence of tones is restricted by a three level finite state grammar [16]. With the tones reflecting target points in the fundamental frequency contour, this model does generate good intonation contours. As the 1980's came to an end, researchers began to focus on more statistical and data-driven models.

Some researchers began to focus on the use of classification and regression trees (CART) to generate intonation. These trees were used with different intonation models to automatically generate fundamental frequency for TTS systems. Classification and regression trees consist of a question on each leaf (node) about the feature. The answers from the tree nodes form another sub-tree path. The leaves of these trees contain statistical measures that define the path taken [14]. The Festival TTS system by Dusterhoff and Black uses classification and regression trees in combination with the Tilt Intonation model to generate prosody. The Tilt Intonation model is used to automatically analyze intonation. There are two types of Tilt events: pitch accents and boundary tones [31]. Unlike previous work which used categorical parameters, the Tilt model uses continuous parameters [31]. Using real speech, information is extracted from the database. Then regression trees are built for each parameter in the Tilt model. These trees are used as the fundamental frequency model for the system.

Neural networks have also been used to automatically generate fundamental frequency. In 1989, Scordilis and Gowdy of Clemson University first used neural networks to generate fundamental frequency. Using a small training corpus of real speech, a network was trained to learn f0 values and f0 fluctuations in phonemes [34]. Similar to the NETTalk and NETSpeak systems, this network used the Back Propagation algorithm to train itself. The network consisted of three layers with a hidden layer of 30 neurons. The results revealed that the network could learn to generate fundamental frequency [34]. In 1992, Traber developed a recurrent neural network to predict the number of pitch values in a syllable. The network was trained using automatically labeled data. The network had two hidden layers with the output representing the 
different f0 values [10]. Recent systems that use neural networks for f0 generations closely follow the system developed in the early 1990's. Today neural networks have been used to automatically generate fundamental frequency for a large range of languages.

\subsection{Back Propagation Algorithm}

The Back Propagation algorithm was first developed around the mid 1970's. Learning is based on the gradient descent in the error [38]. This algorithm consists of two main phases: the forward pass and the backwards pass [38]. This algorithm is very similar to Rosenblatt's Perceptron algorithm and is called the Multi-layered Perceptron.

\subsubsection{Forward Pass}

With the Back Propagation algorithm, the network should first be considered a black box with inputs and a single output.

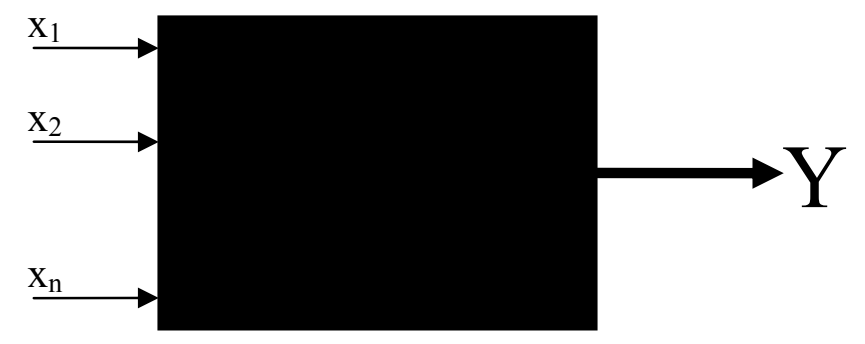

Figure 3-5: The Black Box

Hidden within the black box are neurons in many different layers. The first layers consist of the input neurons, the middle layers are the hidden neurons, and the last layer is the output neurons. Each layer is interconnected to the surrounding layers. 


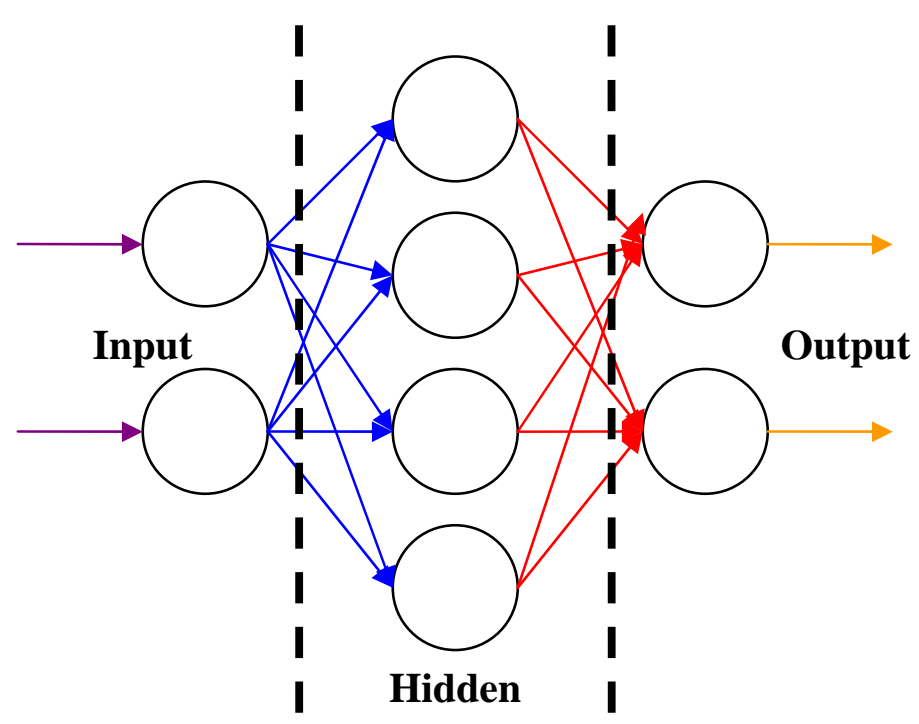

Figure 3-6: Interconnection of Neurons

The outer and input layers are static, while the neurons in the hidden layers can be adjusted. The first step in the Back Propagation algorithm is to create random weights for each neuron in the hidden layers and output layer. The input vector needs to be a vector of ones and zeros. The network is presented with an input vector and a desired output. The hidden weights' outputs are computed using an activation function. The activation function can be sigmoid, tangential, etc.

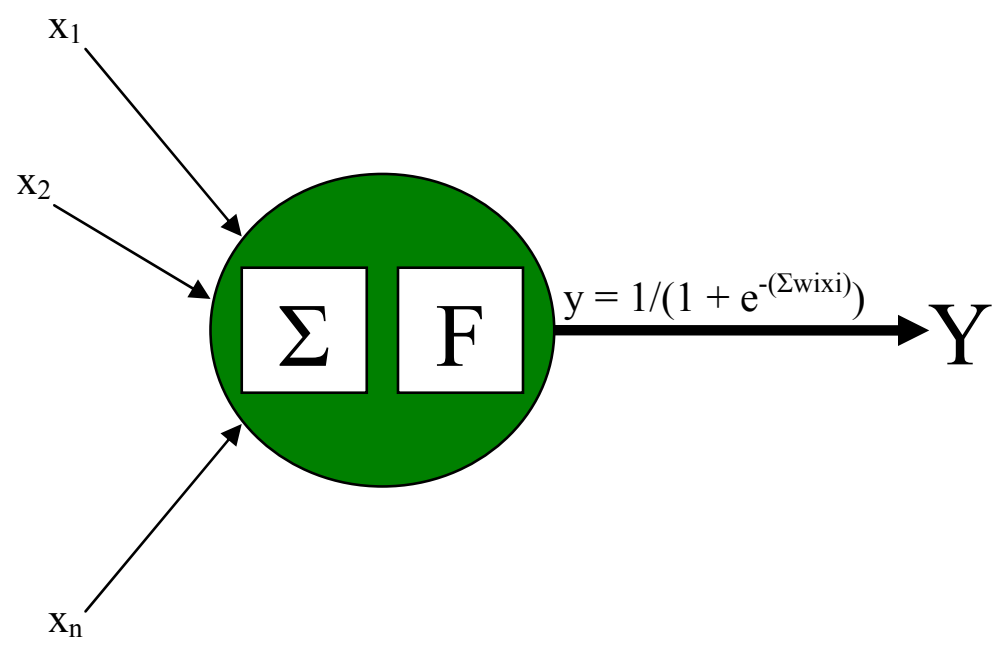

Figure 3-7: The Sigmod Activation Function Neuron 
Using the input vector, the hidden layer neuron weights, and the activation function, the hidden layer nodes' output is computed using the equation:

$y_{k m}=1 /\left(1+\mathrm{e}^{-\Sigma(\text { input vector * hidden neurons weights) }}\right)$

In the equation above, $y_{k m}$ corresponds to the output of the hidden layer. The subscript $k$ represents the number of layers in the system, and the subscript $m$ represents the number of nodes in layer $k$. The hidden layer outputs now become the inputs to the output layer. The output layer nodes' outputs are calculated using the activation function, weights of each output node, and output from each of the hidden layers node.

$y_{(k+1) m}=1 /\left(1+\mathrm{e}^{-\Sigma(\text { hidden layer outputs } * \text { output neurons weights })}\right)$

where $y_{(k+1) m}$ is the output of the output nodes, which is the actual output of the network. Figure 3-8 shows an example of a network and the different inputs and outputs in the layers.

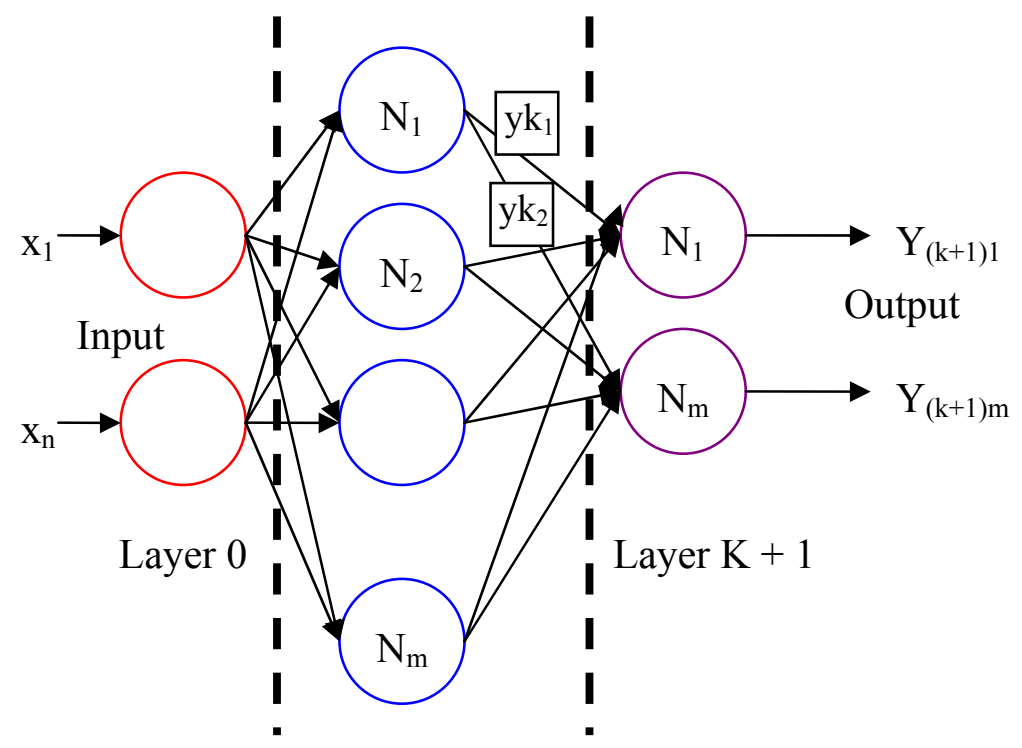

Layer K

Figure 3-8: K layered Back Propagation Network 
Using the actual output and the desired output the Mean Square Error can be determined with the following equation:

$E=\Sigma\left(\mathrm{d}_{\mathrm{m}}-\mathrm{y}_{(\mathrm{k}+1) \mathrm{m}}\right)^{2}$

In this equation, $d_{m}$ is the desired output for the output nodes and $y_{(k+1) m}$ is the actual output of the output nodes. The next phase of the Back Propagation algorithm can now be computed using the Mean Square Error [38].

\subsubsection{Backward Pass}

After the error has been calculated, the weights are updated in each layer to fit more closely to the desired output. The output layer weights are updated using the delta rule equation. This involves changing the weights using the gradient of the calculated error. The error term $\delta$ for the output layer is:

$\delta_{(\mathrm{k}+1) \mathrm{m}}=\mathrm{y}_{(\mathrm{k}+1) \mathrm{m}}\left(1-\mathrm{y}_{(\mathrm{k}+1) \mathrm{m}}\right)\left(\mathrm{d}_{\mathrm{m}}-\mathrm{y}_{(\mathrm{k}+1) \mathrm{m}}\right)$

where $y_{(k+1) m}$ is the actual output at node $m$ and $d_{m}$ is the desired output at node $m$ of the network. Using delta, the weights in the output layer can now be updated thus reducing the error of the network.

$\mathrm{W}_{(\mathrm{k}+1) \mathrm{m}}(\mathrm{t}+1)=\mathrm{W}_{(\mathrm{k}+1) \mathrm{m}}(\mathrm{t})+\eta \delta_{(\mathrm{k}+1) \mathrm{m}} \mathrm{y}_{\mathrm{km}}$

$\mathrm{W}_{(\mathrm{k}+1) \mathrm{m}}(\mathrm{t}+1)$ are the new updated weights in the output layer, and $\mathrm{W}_{(\mathrm{k}+1) \mathrm{m}}(\mathrm{t})$ are the old weights in the output layer. $\eta$ is the learning rate of the network. The learning rate can be a number greater than zero and less than one. If the learning rate is high, the network will converge faster but might be less accurate. However, a low learning rate will cause the network to converge much slower. Also convergence can be changed by using a momentum term $\alpha$ in equation 3.8 .

$\mathrm{W}_{(\mathrm{k}+1) \mathrm{m}}(\mathrm{t}+1)=\mathrm{W}_{(\mathrm{k}+1) \mathrm{m}}(\mathrm{t})+\alpha \eta \delta_{(\mathrm{k}+1) \mathrm{m}} \mathrm{y}_{\mathrm{km}}$

Using $\delta_{(\mathrm{k}+1)}$ from the output layer, the hidden layer error term $\delta_{\mathrm{k}}$ can be computed using the equation: 
$\delta_{\mathrm{km}}=\mathrm{y}_{\mathrm{km}}\left(1-\mathrm{y}_{\mathrm{km}}\right) \Sigma\left(\mathrm{W}_{(\mathrm{k}+1) \mathrm{m}} \delta_{(\mathrm{k}+1) \mathrm{m}}\right)$

In equation 4.7, $\mathrm{y}_{\mathrm{km}}$ are the hidden layer outputs and $\mathrm{W}(\mathrm{k}+1)$ are the updated weights in the output layer. After the error term $\delta_{\mathrm{km}}$ is computed for the hidden layer, the hidden layer weights are updated using $\delta_{\mathrm{km}}$, the presented input vector, and the learning rate.

$\mathrm{W}_{\mathrm{km}}(\mathrm{t}+1)=\mathrm{W}_{\mathrm{km}}(\mathrm{t})+\eta \delta_{\mathrm{km}}$ input vector $_{\mathrm{m}}$

$\mathrm{W}_{\mathrm{km}}(\mathrm{t}+1)$ represents the new hidden layer nodes' weights and $\mathrm{W}_{\mathrm{km}}(\mathrm{t})$ represents the old hidden layer nodes weights. This process continues until some stopping criterion has been met. Once again, convergence can be changed by using the momentum term $\alpha$ in equation 3.11 [38].

$\mathrm{W}_{\mathrm{km}}(\mathrm{t}+1)=\mathrm{W}_{\mathrm{km}}(\mathrm{t})+\alpha \eta \delta_{\mathrm{km}}$ input vector $_{\mathrm{m}}$

\subsection{Fuzzy Logic Inference System}

Fuzzy logic has its roots in philosophy. In ancient Greece, a group of philosophers wrote the "Law of Thought". One of the laws stated that logic must either be true or false. The seeds of fuzzy logic were later planted by Plato who contended that there could be a middle ground between true and false [9]. About 2500 years later, a Polish philosopher named Jan Lukasiewicz described in detail an alternative to the true/false logic of early Greek philosophy. Lukasiewicz mathematically created a tri-valued logic and then later expanded his theory to four-value and five-value logic. Lukasiewicz's work affirmed that someday logic could be expanded to infinite-value logic [9].

The father of modern fuzzy logic created this infinite-value logic. In 1965, Professor Lotfi Zadeh of UC Berkeley wrote the paper "Fuzzy Sets" which described, mathematically, the theory of fuzzy logic. In this paper, Zadeh described the two opposites, true and false, as membership functions. These membership functions truths are determined through a range of numbers, normally between 0 and 1 [42]. In 1973, Zadeh expanded on this theory, solving complex systems and decision processes [9]. 
Fuzzy systems are designed to take advantage of imprecision. Solving real world problems with precise logic can be difficult. Logic deals with making an absolute choice, like true or not true. But sometimes the right choice is somewhere in between true and not true. Fuzzy systems add fuzziness to the choice in order to accommodate the grey area of logic.

Fuzzy systems assign truth values to statements to determine their truthfulness. These numbers are usually between 0 and 1 . The truth value determines the membership of a certain group. To describe these groups, the fuzzy system uses the membership function. With a given statement, the membership function determines the truthfulness of that statement. The membership function can be many shapes and sizes. Some examples of membership functions are shown in Figure 3-9.
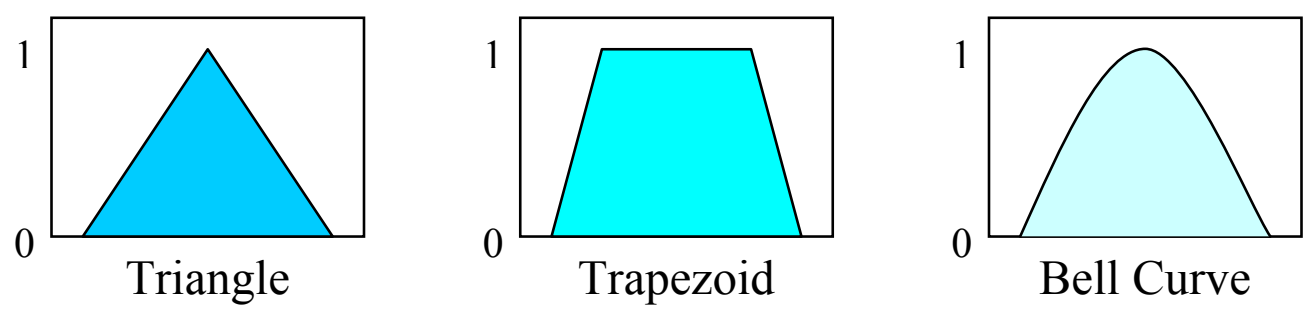

Figure 3-9: Different Types of Membership Functions

The fuzzy system is built with linguist rules and membership functions. The linguist rules are used to conduct the actions of the fuzzy system. The membership functions are used to determine how these rules affect the fuzzy system. The rules are built using the logical terms like, $O R, A N D, N O T$, and THEN. Rules can also be built with other terms to hedge behavior like, MORE, LESS, VERY, and SOMEWHAT. Some examples of how these terms can be used to build rules are:

"If Jon is tall OR Jon is fat THEN Jon's size is big."

"If the car is old $A N D$ the car is cheap THEN the car is worthless"

"If the car is NOT old $A N D$ the car is NOT cheap THEN the car is valuable"

Each term used in the fuzzy system has different effects on the behavior of the system. The $O R$ term is the same as the $U N I O N$ of two variables. The $O R$ term is equivalent to the maximum. The $A N D$ term is the equivalent to the INTERSECTION of 
two variables. The $A N D$ term is equivalent to the minimum. The NOT term corresponds to the COMPLEMENT of a variable which is its opposite. Finally, the THEN term indicates the consequence produced by the rule.

The descriptive linguist variables in the rules represent the membership of the statement. Therefore, descriptive linguist variables are the membership functions of the fuzzy system. The fuzzy system is constructed of different fuzzy inputs and consequences of the inputs. Each input and consequence of the fuzzy system has its own set of membership functions. When an input is presented to the fuzzy system, the output is determined by the firing strength of the rules onto the input. From the rules and the presented input, a crisp output is calculated to produce the output of the fuzzy system. 


\section{Chapter 4: Text-to-Phoneme Conversion}

\subsection{Text-to-Phoneme Conversion Overview}

This chapter presents the algorithm used to convert user's text into phonemes. Phonemes are the smallest phonetic unit in a language within a word. When American students first learn to read, they are taught all of the English phonemes. Table 4-1 gives some examples of phonemes and the sounds that they represent.

Table 4-1: Examples of English Phonemes

\begin{tabular}{|l|l|}
\hline Phoneme & Sound \\
\hline$/ \mathrm{p} /$ & $\underline{\text { pit }}$ \\
\hline$/ \mathrm{k} /$ & $\underline{\text { cat }}$ \\
\hline$/ \mathrm{t} /$ & $\underline{\text { tap }}$ \\
\hline$/ \mathrm{a} /$ & $\underline{\text { about }}$ \\
\hline$/ \mathrm{e} /$ & bend \\
\hline$/$ ä/ & father \\
\hline$/ \mathrm{sh} /$ & $\underline{\text { show }}$ \\
\hline$/ \mathrm{zh} /$ & measure \\
\hline
\end{tabular}

In order to convert text into speech, the algorithm must be able to handle words in the English language that do not sound like the way they are written. The method used to accomplish this task is neural networks.

Neural networks have been used to solve many types of problems over the years. These problems include pattern recognition, data classification, and other very complex problems. The advantage of neural networks is in their parallel architecture. The neural network simulates how the brain uses interconnected neurons to process information. With neural networks, the nodes of the networks are interconnected and work in unison to solve specific problems. However, the only way the neuron network learns is by 
example. Therefore, the network must guess an output, compute an error using the actual output, and then correct itself by adjusting its neurons [38].

Converting English text to English speak is an example of a complex problem that neuron networks can solve. English text can be very tricky to pronounce. For example, words like "Philadelphia", "bike", and "brought" are not pronounced how they are spelled. Using data to correct itself, a network can learn to pronounce English text. For this system, a neural network is trained with the Back Propagation algorithm using a data set of about 1800 words [38]. The network was trained on a Dell Optiplex $2.80 \mathrm{GHz}$ Intel Pentium 4 computer.

\subsection{Text-to-Phoneme Conversion Algorithm}

The TTP algorithm used by this system follows very closely to the algorithm used by Sejonowski and Rosenberg. Sejonowski and Rosenberg created the NETTalk system in the late 1980's. As previously discussed in Section 3.2, this system used the Back Propagation algorithm to train a network to pronounce inputted text. The original NETTalk used a three layered network with 120 hidden neurons and 26 output neurons that represented each phoneme. Figure 4-1 shows the outline of the NETTalk program. The training data used in the NETTalk system consisted of 2000 of the most common words aligned with their pronunciations [35]. Although this algorithm is similar to the

original NETTalk algorithm, there are some slight modifications to the network, training data, and training algorithm.

The overall network should be thought of as a loop. An input is taken from the training data, an output is calculated, and the nodes of the network are adjusted according to the error. Then another input vector follows the same steps, and the process continues until some stopping criterion is met. 

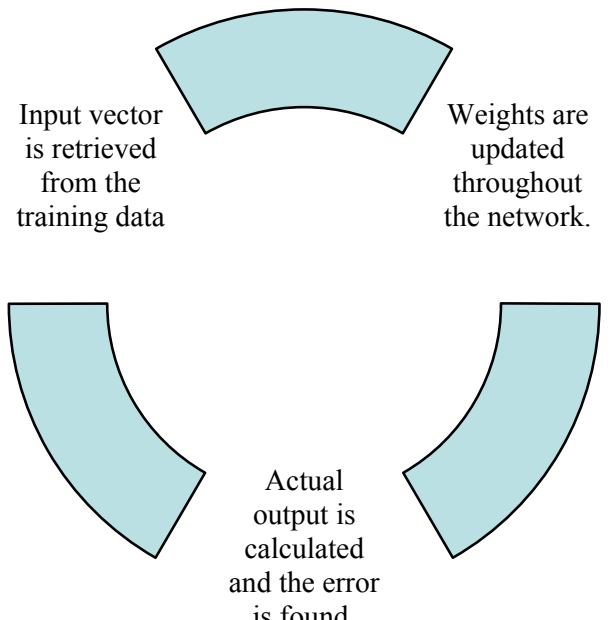

Figure 4-1: Network Training Cycle

\subsubsection{Network Input and Output Design}

The network consists of seven windows for the input and one output for the system. The input to the network is not the total word but single characters. Each word from the training data is entered into the window one character at a time. The inputted training word enters the system and moves through the seven windows one character at a time. The network's input is the seven windows. Figure 4-2 shows an example of how this process takes places.

Network Loop 1
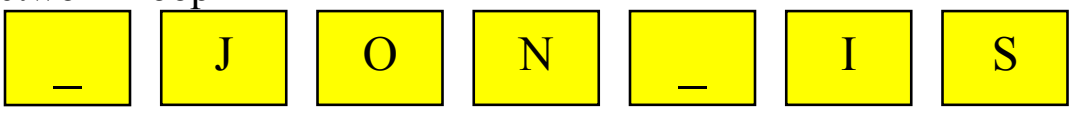

Network Loop 2
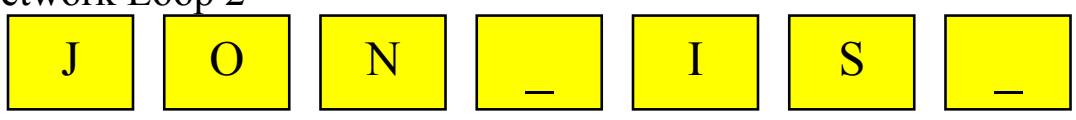

Network Loop 3
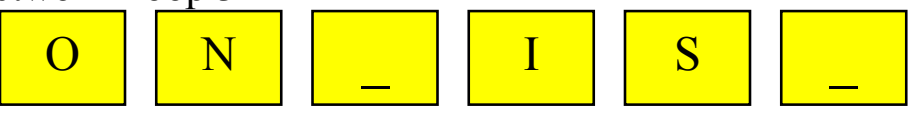

\section{C}

Figure 4-2: Moving Window Example 
The output of the network is the phonetic representation of the $4^{\text {th }}$ input window at any given time. The input and output must be aligned to ensure that the output represents the correct input window. The output can also be thought of as seven windows aligned with the seven windows of the input. However, the only output window of concern is the $4^{\text {th }}$ window. As the input letters move through the seven input windows, the output phonemes move through the seven output windows. During training, the network inputs are all seven letters in the input windows, and the network desired output is the phoneme in the fourth position of the output windows. Figure 4-3 shows an example of how the output windows work.

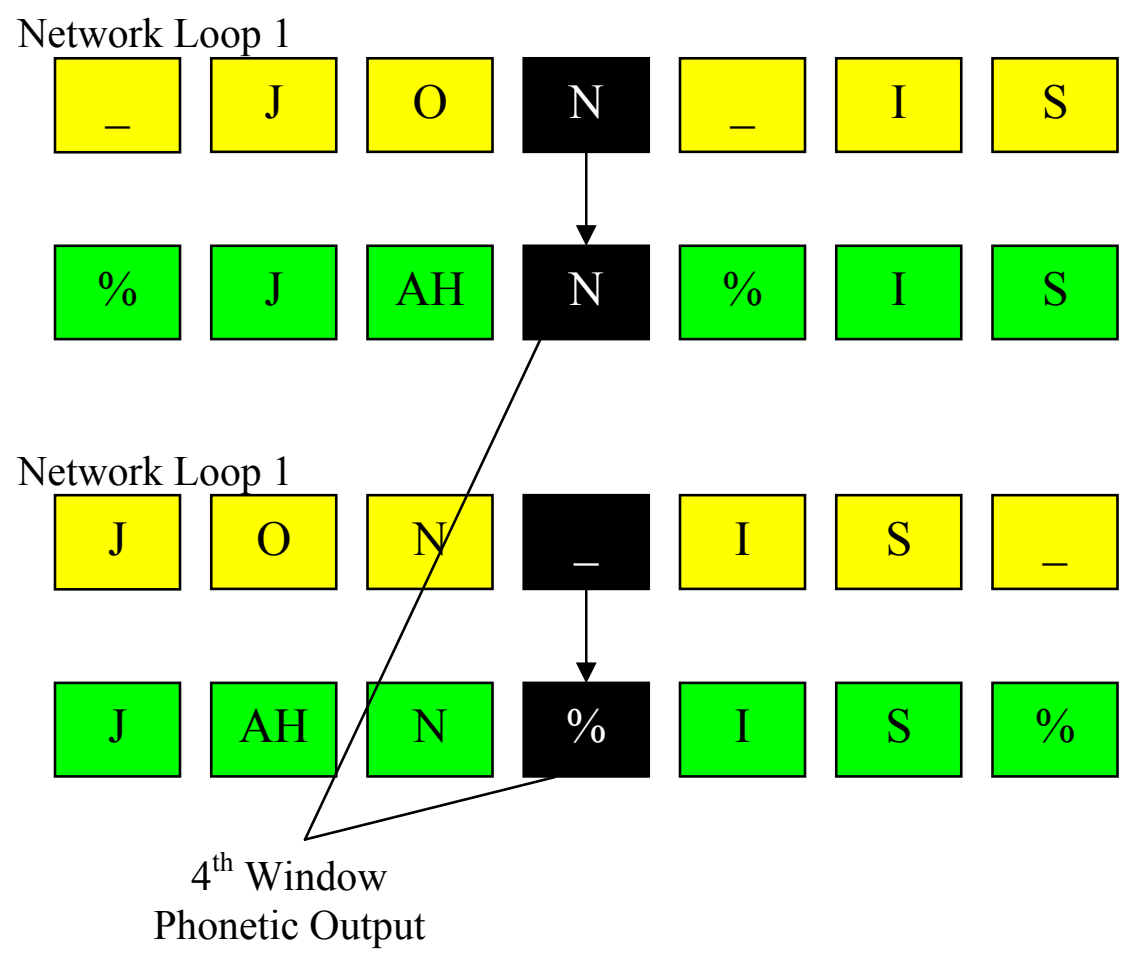

Figure 4-3: Moving Window Example

\subsubsection{Network Detailed Training Design}

The training of the network involves obtaining a word and its phonetic representation from the training set, placing the word's first character in the first input window, calculating the actual output of the seven windows, and updating the weights. The network then moves the characters throughout the windows until the word ends. If 
training is not done, the network retrieves the next word from the training set. Figure 4-4 shows an outline of the training algorithm.

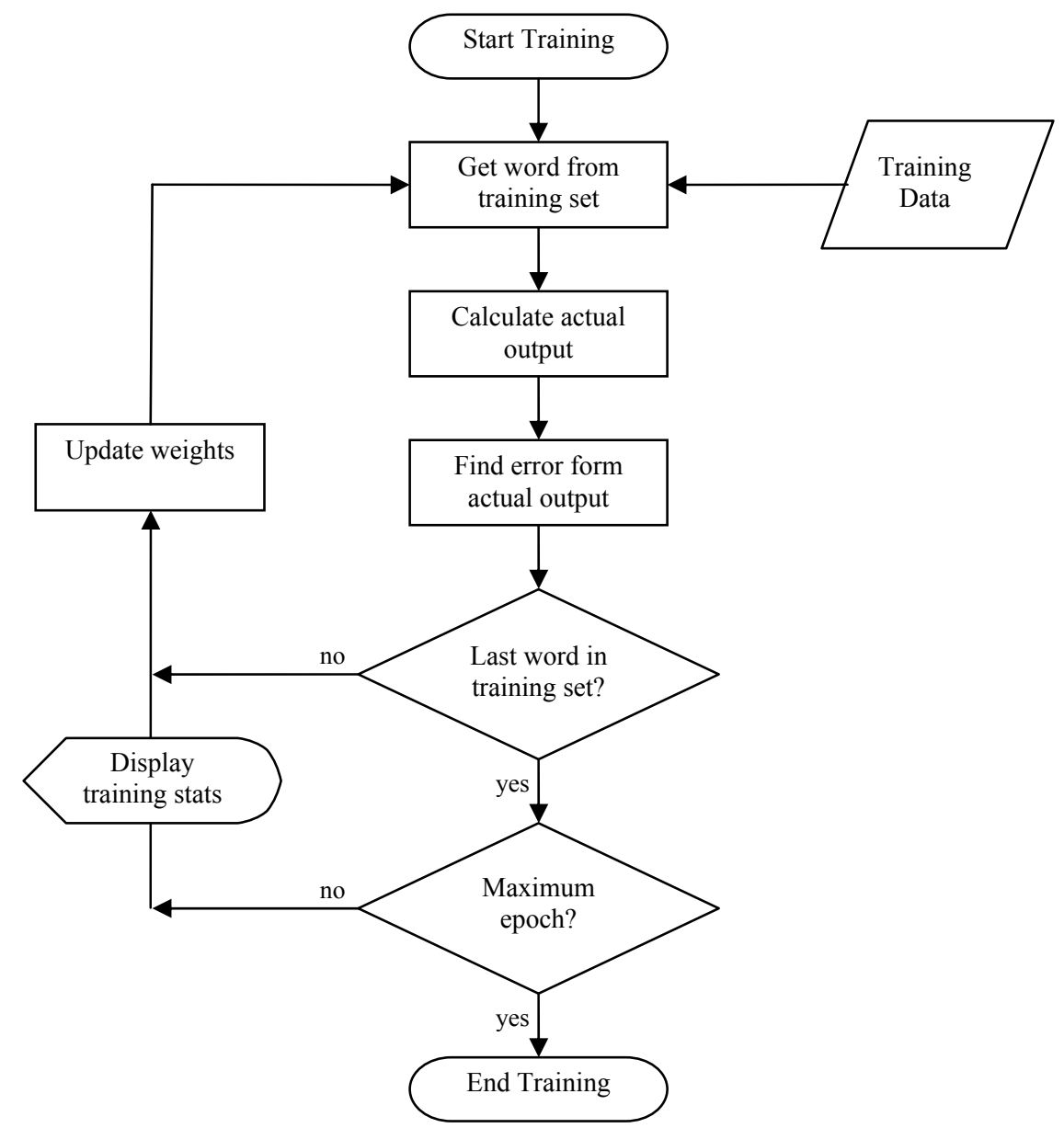

Figure 4-4: Network Training Flow Chart

The first step of the network is retrieving the input vector from the training data. However, the network does not start with the first word of the data when training first begins. Initially, the training network input is set to all silences with the desired output being a silence. This was performed to simplify the beginning of the training process and to insure that the network and the output are aligned properly. Figure 4-5 shows the initial look of the network at the beginning of training. 


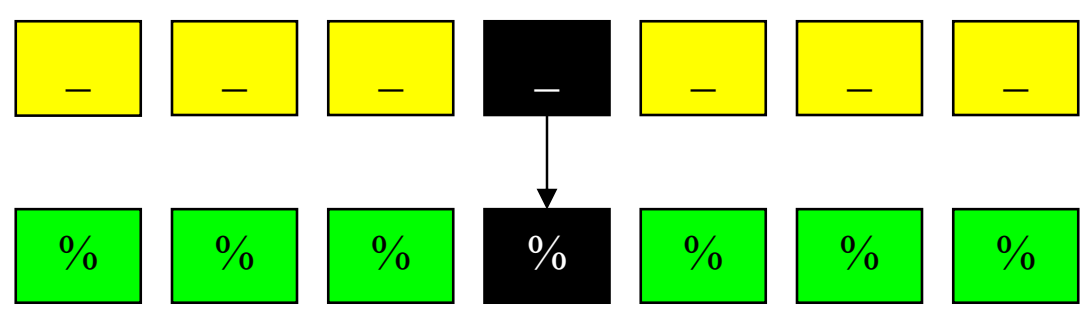

Figure 4-5: Initial State of the Network

After the first iteration, the first word is retrieved from the training set. The first letter of the word is placed in the seven letter window. If the first word is "aardvark", then the seven windows would have six silences and one letter after the first iteration. The desired output would still be a silence. Figure 4-6 shows the seven input windows with the desired output.

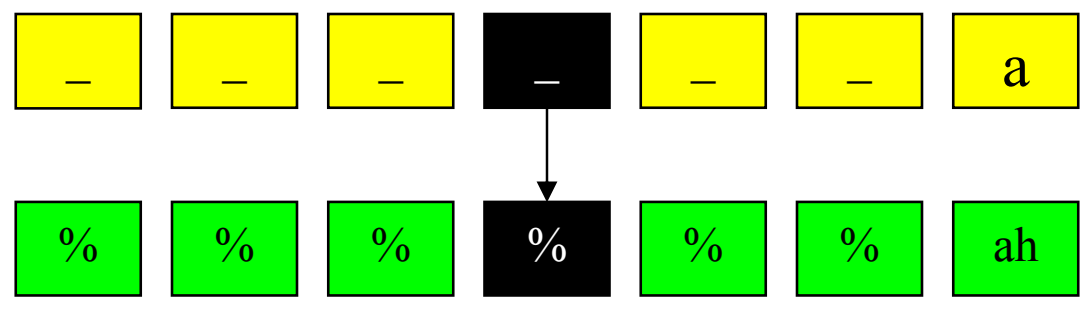

Figure 4-6: State of the Network after First Iteration

In order to calculate the actual output of the system, the letters and phonemes need to be converted to numbers. In this program, each input letter is converted into a vector. The vector size is the total number of characters used in the network. For the network, there are 27 characters used for the input, 26 characters that represent the alphabet and one character that represents a silence. The input vector consists of zeros and a one. Each input vector is all zeros, with a one located to indicate the letter. For example, the letter $d$ would be a vector of zeros with a one located at the $4^{\text {th }}$ position of the vector. 


\begin{tabular}{|c|c|c|c|c|c|c|c|c|c|c|c|c|c|c|c|c|}
\hline 1 & 2 & 3 & 4 & 5 & 6 & 7 & 8 & 9 & 10 & 11 & & & & 25 & 26 & \\
\hline 0 & 0 & 0 & 1 & 0 & 0 & 0 & 0 & 0 & 0 & 0 & 0 & - & 0 & 0 & 0 & 0 \\
\hline
\end{tabular}

Figure 4-7: Input Vector for the Letter d

The output vector is very similar to the input vector. The vector size is the total number of different phonemes used in the network. There are 41 phonemes used, 40 for sound and one for silence. The ideal output consists of all zeros and a one; therefore, the network is trained using a desired output vector with all zeros and a one. Each phoneme has its own location in the output vector. Like the input vector, the one is located where the phoneme is located in the vector. For example, a desired output phoneme $/ a h /$ would produce a desired output vector of all zeros and a one placed in the $2^{\text {nd }}$ position of the vector.

\begin{tabular}{|c|c|c|c|c|c|c|c|c|c|c|c|c|c|c|c|}
\hline 1 & 2 & 3 & 4 & 5 & 6 & 7 & 8 & 9 & 10 & 11 & & 3 & 39 & 40 & 41 \\
\hline 0 & 1 & 0 & 0 & 0 & 0 & 0 & 0 & 0 & 0 & 0 & 0 & 0 & 0 & 0 & 0 \\
\hline
\end{tabular}

Figure 4-8: Desired Output Vector/ah/

Once the letters are converted to vectors, the actual output can be calculated. During training, the output vector is computed using all seven windows. Therefore, the input used for calculation is a $7 * 27$ long vector with seven ones located throughout the vector. All the weights of the network, i.e. hidden weights and output weights, are initially set to random values between -.5 to .5 . Using the equations from Section 3.5.1, the actual output is calculated.

hidden layer vector $=1 /\left(1+\mathrm{e}^{-\Sigma(\text { seven window input vector } * \text { hidden neurons weights })}\right)$

actual output vector $=1 /\left(1+\mathrm{e}^{-\Sigma(\text { hidden layer vector * output neurons weights })}\right)$

These equations will produce an output vector output of the network. The actual output and the error of the actual output are calculated. Weight adjustment occurs after each 
loop, i.e. after every single letter of every word in the training set. The weights of the system are changed using the backward propagation equations described in Section 3.5.2.

output layer weights $=\mathrm{W}_{\text {old output }}+\alpha \eta \delta_{(\mathrm{k}+1) \mathrm{m}}$ hidden layer ouput

hidden layer weights $=\mathrm{W}_{\text {old hidden }}+\eta \delta_{\mathrm{km}}$ input vector $_{\mathrm{m}}$

Once the weights are adjusted, the network will retrieve the next letter and desired output of the word. If there are no letters left in the word, the network will retrieve the next word from the training set. Once the last letter of the last word is retrieved for the training set, an epoch has been completed and the average error is calculated and displayed. The next word entered into the network would then be the first word of the training set. Training will continue in this manner until the maximum number of epochs is reached. Once the training is complete, the weights of the network are saved, and the weight can be used for TTP mapping.

\subsubsection{Training Set Detail and Alignment}

Training the network involves using real words aligned with their exact pronunciation phonemes. For example, 'cat' or 'example' would be aligned with 'kat' and 'eksampul', respectively. The list of the 41 phonemes used for this system is in Appendix A. Originally, about 900 words were used from the "Cue Practice With the 1000 Most Common Words" website as training data. However, this amount of data was not sufficient enough to yield an acceptable performance. The rest of the data was chosen based on the network performance using www.allwords.com. For example, the network initially struggled with words beginning with "th", so about 20 words beginning and ending with "th" were added to the training data. Appendix $\mathrm{C}$ lists the entire training set.

Certain factors need to be accounted for when choosing the training data. The network input size consists of only seven windows. However, since the words are entered into the training window one letter at a time, the word size can be any number of characters. In addition, all words, even the words initially found on the web, need to be aligned by hand in order to maximize the performance of the system. Figure 4-9 shows an example of how this alignment is done. 


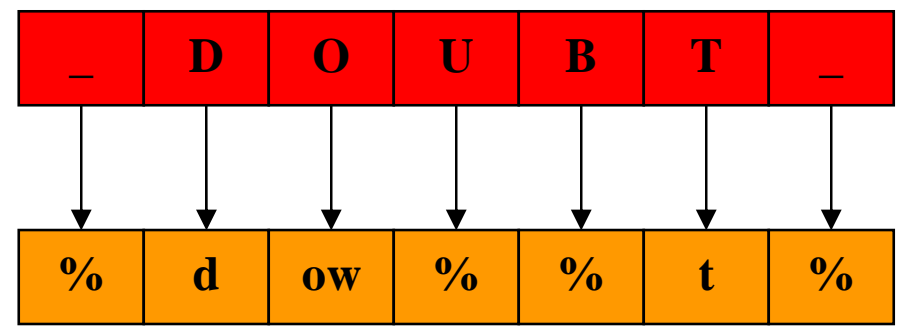

Figure 4-9: Word Alignment 


\section{Chapter 5: Automatic Prosody Generation}

\subsection{Generation of Prosody Overview}

Prosody deals with the tone and the timing of speech. In speech synthesis systems, the most complex problem is the generation of natural sounding prosody. Real speech often reflects the speaker's personal knowledge of the audience, the mood and emotional state, the general knowledge of the world around them, and the reaction from the audience. With TTS systems, this problem is more complex because the text input is random. These issues have yet to be resolved by speech synthesis systems. Automatic prosody generation has to factor in these setbacks and focus on neural language that is understandable to the audience.

The main focus of this thesis is the automatic generation of prosody. This system generates two main aspects of prosody: fundamental frequency and segmental duration. Fundamental frequency is generated using fuzzy logic. The primary focus of this chapter will be on this aspect of prosody. Segmental duration is determined using rules described by Klatt [2]. After the network converts the text to phonemes, the system then computes the prosody of the system. Both the phonemes found by the network and the actual texts are used to generate prosody. The prosody generator can be thought of as a finite loop, with information describing each phoneme used to produce both duration and fundamental frequency. The first step of prosody generation is segmental duration.

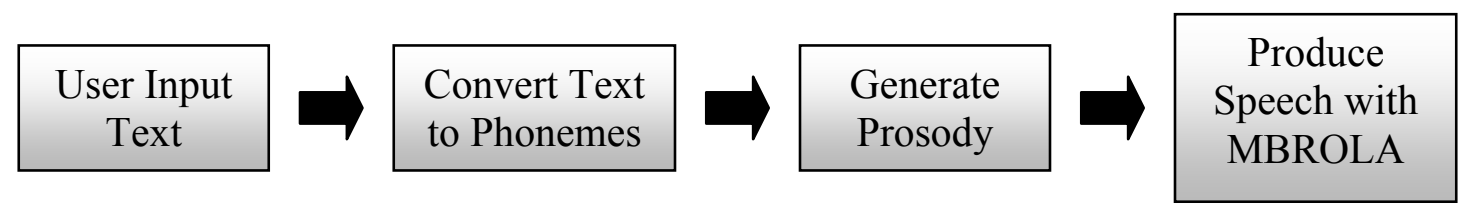

Figure 5-1: System Overview 


\subsection{Segmental Duration}

Segmental duration handles the length of segments within words. These segments can be either syllables or phonemes. For this system, the focus will be on phoneme duration. Researchers believe that English phoneme durations are only affected by a few parameters. In 1979, Dennis Klatt developed rules that governed the durations of phonemes within words. These rules were used in the aforementioned MITTalk TTS system [2]. The Klatt Duration Rules are used to generate the segmental duration of this system.

The original MITalk system was a formant synthesized speech. With formant synthesizers, certain factors need to be taken into account. Phonemes with different articulations are handled differently when it comes to duration. For example, the duration of a fricative is measured by the visible noise. For stops, the duration also includes the closure [2]. With this TTS system, the speech synthesis is generated using diphone concatenation. Therefore, these factors are not factored into consideration when computing the segmental duration.

The Klatt Duration rules were designed to replicate observed duration from a speaker. There are two main rules that govern the model: segments that are altered by a percentage using the rules and segments that cannot be shorter than a minimum duration. Each segment has its own minimum and average duration. The minimum and average durations for each phoneme used in this system are shown in Appendix B. The formula used to alter the phoneme duration is:

DURATION $=((A V E D U R-M I N D U R) *(P R C N T)) / 100+$ MINDUR

The variable $A V E D U R$ is the average duration, and variable MINDUR is the minimum duration. The Klatt rules work by altering the PRCNT variable. There are a total of 11 Klatt Duration rules. Each rule has a justification on how the rule was created. The rule formulas were developed through extensive trial and error. The Klatt duration rules are listed below. These rules are obtained directly from the book, "From Text to Speech: The MITalk System" [2]. 
1. Pause insertion Rule

Insert a 200 millisecond pause before each sentence-internal main clause and at boundaries delimited by a syntactic comma, but not before relative clauses.

2. Clause-final lengthening The vowel or syllabic consonant in the syllable before a pause is length by PRCNT $=140$.

3. Non-phrase-final shortening

Vowels and syllabic consonants are shortened by PRCNT $=60$, if not in the phrase's last syllable. A phrase final postvocalic liquid or nasal is lengthened by PRCNT $=140$.

4. Non-word-final shortening

Vowels and syllabic consonants are shortened by PRCNT $=85$, if not in the word's last syllable.

5. Polysyllabic shortening

Vowels and syllabic consonants in word with multiple syllables are shortened by $\mathrm{PRCNT}=80$.

6. Non-initial-consonant shortening

Consonants are shortened by PRCNT $=85$, if not in the word's initial position.

7. Non-phrase-final shortening

Vowels and syllabic consonants are shortened by PRCNT $=60$, if not in the phrase's last syllable. A phrase final postvocalic liquid or nasal is lengthened by PRCNT $=140$.

8. Lengthening for emphasis

Emphasized vowels are lengthened by PRCNT $=140$.

9. Postvocalic context of vowels

The consonant after a vowel in the same word influences the length of the vowel. The list below shows these effects.

- $\quad$ open syllable, final word PRCNT1 $=120$

- $\quad$ before a voiced fricative PRCNT1 $=160$

- $\quad$ before a voiced plosive PRCNT1 $=120$

- $\quad$ before a nasal PRCNT1 = 85

- $\quad$ before a voiced plosive PRCNT1 $=70$

10. Shortening Clusters

Segments are shortened in consonant-consonant sequences and in vowel-vowel sequences.

- $\quad$ vowel followed by a vowel PRCNT1 $=120$

- $\quad$ vowel proceeding a vowel PRCNT1 $=70$

- $\quad$ consonant surrounded by consonants PRCNT1 $=50$

- $\quad$ consonant proceeding a consonant PRCNT1 $=70$

- $\quad$ consonant followed by a consonant PRCNT1 $=70$

11. Lengthening due to plosives aspirations

A primary or secondary stressed vowel or sonorant preceded by a voiceless plosive is lengthened by 25 milliseconds. 


\subsection{Stress Assignment}

One of the most crucial components of generating automatic prosody is stress assignment. Stress assignment is placing lexical stresses on the proper syllable. All stresses are placed on vowels. With TTS synthesis this can be very complicated. Since the input is random, the words are random. Therefore, a stress assignment algorithm needs to be implemented that is both automatic and flexible. The stress assignment algorithm used for this system is the rule-based system used in the MITalk. Those rules were based on the Halle and Keyer lexical stress rules developed in 1971 [2].

The rules are based upon phonetic input without regard to part-of-speech. Stresses are placed on individual words independently. There are three levels of stress; 0stress, 1-stress, and 2-stress. The 1-stress represents the primary stress in a word and the 2 -stress represents lesser stresses. 0-stress represents no stress. The stress rules have two different phases. The first phase is called the cyclic and is committed to placing primary stresses on the word. There are three rules in the cyclic phase. The first rule in the cyclic phase is the main stress rule, and the other two rules are exceptions to the first rule. The second phase is called the non-cyclic phase and includes the application of the entire word of rules. The non-cyclic phase reduces the final word to just one 1-stress mark and turns the rest of the primary marks into 2-stress marks [2].

\subsection{Fuzzy Fundamental Frequency}

Fundamental frequency is a vital part of the naturalness of any TTS system. This frequency, sometimes called f0 frequency, produces the tone of speech. An example of fundamental frequency can be heard in the difference between a male and a female voice. Male voices exude a lower overall fundamental frequency while female voices typically have a higher overall fundamental frequency. Fundamental frequency can be generated in many ways for TTS systems. Most systems today generate fundamental frequency using unit selection synthesis together with pre-recorded units (sounds). These prerecorded sounds contain natural frequency fundamentals. Other methods used to generate frequency fundamentals are rule-based and neural network approaches. 
The fundamental frequency of this TTS system is generated using the flexibility of fuzzy logic. Fuzzy logic can be used to control complicated problems. The generation of fundamental frequency for TTS systems is a very complex problem for many reasons. For example, the user's input needs to be thought of as random; therefore, the system must be flexible. Also, the fundamental frequency in speech is different for every sentence, and many factors control this overall curve. Fuzzy logic can be a solution to the fundamental frequency problem by using a small set of rules. The rules are constructed based upon the fundamental frequency algorithm used in the MITalk system, the O'Shaughnessy algorithm.

\subsection{O’Shaughnessy Algorithm}

The O'Shaughnessy fundamental frequency algorithm was used in the MITalk system to generate fundamental frequency. The result of the algorithm is a fundamental frequency curve throughout the sentence. This curve is called the f0 contour. This algorithm is detailed in the book, "From Text to Speech: The MITalk System" [2]. The algorithm has two main levels, high and low, which work as two different systems. The first level is the high level. This level generates an outline of how the overall f0 contour should be shaped. The high level of this algorithm takes grammatical information about the sentence and builds a basic contour of the system. The fo contour in the high level is augmented using four factors: sentence type, phrase contour, word contour, and prosodic indicators [2].

The first factor, sentence type, is determined by the first word and punctuation of the sentence. For example, the endings of questions are indicated by question marks and the endings of statements are indicated by commas or periods. There are three tunes that represent three types of sentences: declarative sentences, yes/no question and "wh"

questions. These three tunes determine the f0 contour trend of the sentence. Every tune has a downward linear trend throughout the sentence [2]. Figure 5-2 shows this trend. 


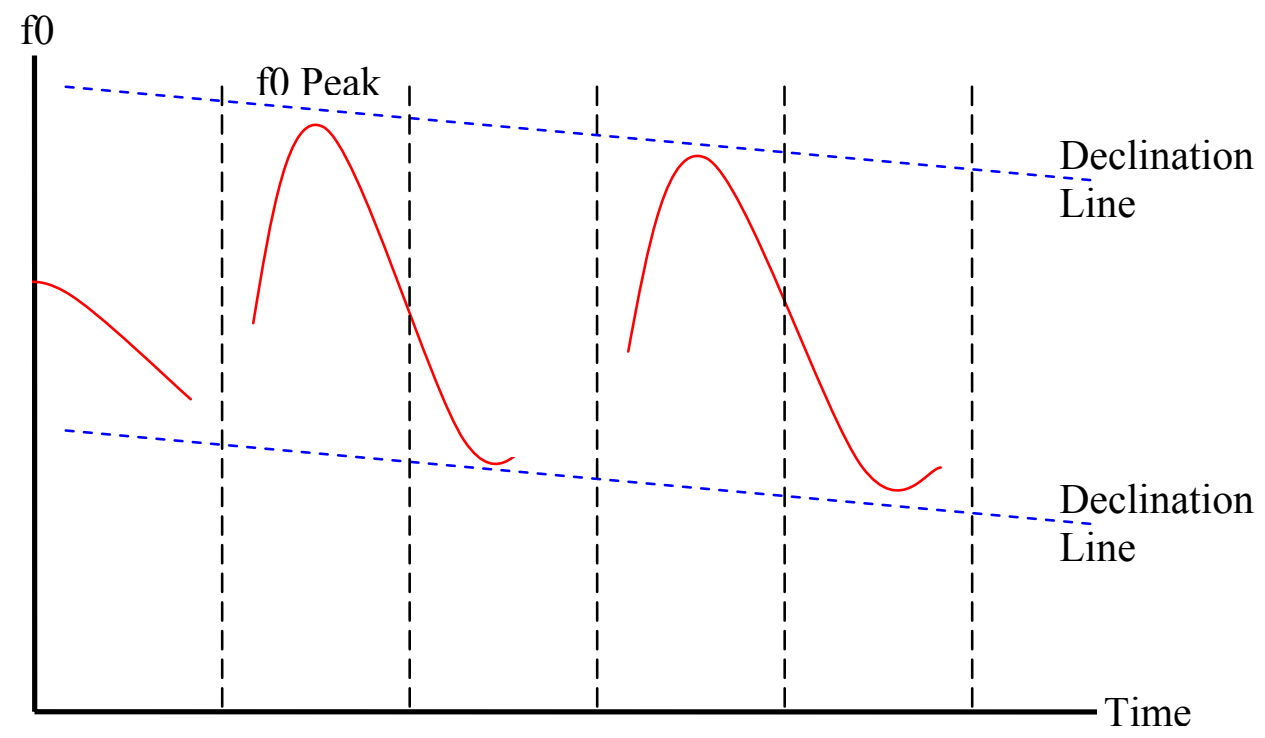

Figure 5-2: Downward Linear Trend

Tune A, which deals with declarative sentences, causes the f0 contour to fall linearly throughout the sentence with the sharp decline at the end of the sentence or phrase. Tune B manages yes/no questions. Tune B sentences have an initial rise to the f0 contour, followed by a flat contour with a final sharp rise. The final tune, which handles "wh" questions, starts with a high peak on the "wh" word, followed by a steep fall and a high rise at the end of the sentence [2].

The second factor of the algorithm contends with the phrase contour of the sentence. The different phrases of a sentence can be determined with many different algorithms. Each phrase must have two or more content words to be affected by this factor. At the beginning of each phrase, the f0 curve rises sharply on the first content word. At the end of each phrase, the f0 curve falls on the final content word.

The third factor handles the individual words in a sentence. Within each word, the $\mathrm{f} 0$ contour fluctuates. This third factor determines the amount of change within each word. The amount of change is correlated with the importance of the word and the amount of syllables in the word [2]. Words that are more important and have a great deal of syllables contain the most f0 changes. Table 5-1 shows the importance of these words. 
Table 5-1: Word Importance

\begin{tabular}{|c|l|}
\hline Level & Part of Speech \\
\hline 0 & article \\
1 & conjunction, relative pronoun \\
2 & preposition, auxiliary verb \\
3 & personal pronoun \\
6 & verb, demonstrative pronoun \\
7 & noun, adjective, adverb, contraction \\
8 & reflexive pronoun \\
9 & stressable modal \\
10 & quantifier \\
11 & interrogative adjective \\
12 & negative element \\
14 & sentential adverb \\
\hline
\end{tabular}

The prosodic indicator assignment is the last factor of the high level part of the algorithm. First, accent numbers are given to each accent in the sentence. The number depends on the size and importance of the word. Then an integer that represents the word position is placed on each word. Words at phrase boundary positions are given larger integer values than words that are in the middle of the phrase. The high level part of the algorithm combines all of these factors to form the outline of the sentence fo contour. The high level then becomes the input to the low level component of the algorithm [2].

The low level of the O'Shaughnessy algorithm sculpts the details of the final f0 contour. In the low level, the f0 contour is affected by the number of syllables in combination with the lexical stress. The importance of the word affects the height of the stressed peak. More important words have higher peaks on their stresses. The amount of syllables directly influences the fundamental frequency of the sentence. The first and highest peak should have an f0 contour of about $190 \mathrm{~Hz}$. However, larger sentences with more syllables have a higher starting peak frequency, and smaller sentences with fewer syllables have a lower starting peak frequency. The number of syllables between stressed syllables also affects the height and outer shape of a peak. Stressed peaks separated by two or three syllables have their peak height decreased by $15 \%$ and $20 \%$. Stressed peaks separated by two, three, or four syllables have their peak rise increased by $15 \%, 20 \%$, and $30 \%$, respectively. Stressed peaks that are adjacent have their peak rises decreased by $40 \%$. All other peaks maintain their heights and are shaped by a default rise [2]. 


\subsection{Fuzzy System Inputs and Consequence}

The fuzzy system is developed to model the O'Shaughnessy algorithm using a fuzzy interference system. The inputs to the fuzzy system are the phonemes' linguistic data. The output of the fuzzy system is the fundamental frequency. The system consists of four inputs and one output. The inputs are: word importance, sentence size, position in sentence, and distance from stress. Each input has three linguistic variables and the output has seven linguistic variables. Using a system similar to the system described in 4.3.2, the fundamental frequency can be computed for any given sentence.

\subsubsection{Word Importance}

The inputs to the fuzzy system are formulated from the main parts of the O'Shaughnessy algorithm. The first input is the importance of the word. This input comes from the word importance of the O'Shaughnessy algorithm. The O'Shaughnessy algorithm places importance on words based on part-of-speech. There are two types of words this system takes into consideration, function words and content words. Content words have more importance than function words, with articles having the least importance. The system calculates the importance of the word based on the size of the word and the type of word. The calculation yields a number between 0 and 10; therefore, the fuzzy input consists of a number range between 0 and 10. The Word Importance input has three linguistic variables for this input: useless, semi-important, and important. Figure 5-3 shows the membership functions of the Word Importance input.

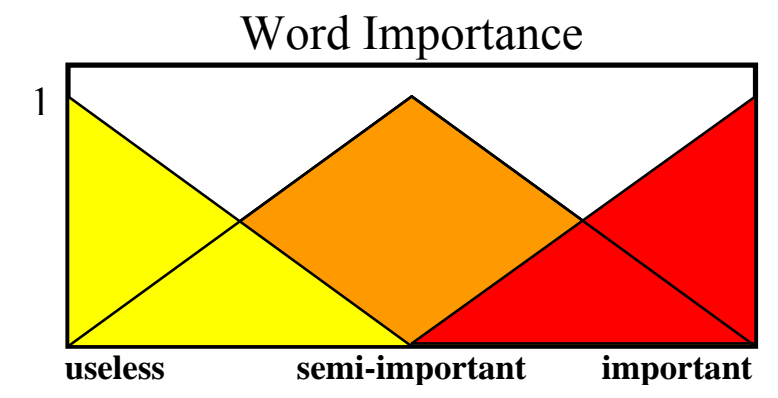

Figure 5-3: Word Importance Input Membership Functions 


\subsubsection{Sentence Size}

The second input to the fuzzy system is the sentence size. In the O'Shaughnessy algorithm, peak size is correlated with the length of the sentence. According to the algorithm, smaller sentences, i.e., sentences with fewer syllables have smaller fundamental frequency peaks. Larger sentences have much larger fundamental frequency peaks. The input is divided into three linguistic variables: small, medium, and large. The sentence size is determined by the number of syllables multiplied by an offset.

Size $=$ (number of phonemes) $*$ offset

offset $=.5$

The input range values are from 0 to 10 . Any size that is greater than 10 is considered to be equal to 10 by the system.

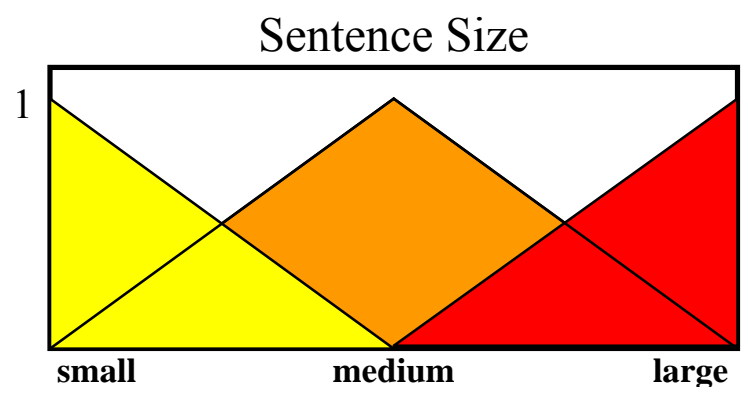

Figure 5-4: Sentence Size Input Membership Functions

\subsubsection{Sentence Position}

The third input is the position in the sentence. The position in the sentence deals with the word, not the phoneme. Sentence position is calculated using the following equation:

Position $=($ word location in sentence / total number of words $) * 10$ 
The location of the word in the sentence is a very important aspect of the O'Shaughnessy algorithm. As described in Section 5.5, each tune's fundamental frequency contour differs throughout the sentence. Therefore, the location of the phoneme or word determines the frequency contour depending on the type of tune. For example with tune B sentences (yes/no questions), at the end of the sentence, the fundamental frequency increases sharply. Like the previous inputs to the fuzzy system, the Sentence Position input has three linguistic variables and a number range between 0 and 10. The input variables are start, middle, and end. Figure 5-5 shows the membership functions for the Sentence Position input.

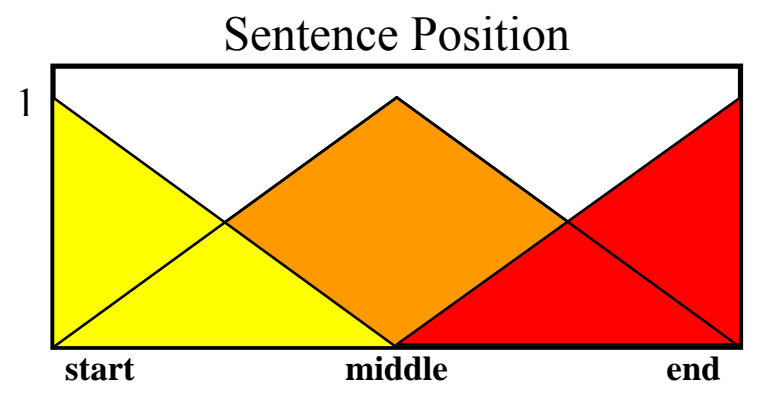

Figure 5-5: Sentence Position Input Membership Functions

\subsubsection{Stress Distance}

The final input to the fuzzy system is the phoneme distance from the stress. The O'Shaughnessy algorithm places peaks on primary stresses in sentences. The fundamental frequency of phonemes around these stresses is also altered. Peak size depends on the location, sentence type, and importance of the word. Stress distance is calculated using the closest, right-hand, most stress within the word. If a phoneme is stressed, its distance value is "dead-on". Stressed phonemes equal the maximum. Phonemes inside of a word without a stress are assigned a zero. The fuzzy input ranges from 0 to 10 . If the phoneme is not stressed and the word contains a stress, the distance is calculated using the formulas:

stress distance $=(($ stress location - phoneme location $)+1) *$ offset offset $=10 /$ (length of the word) 


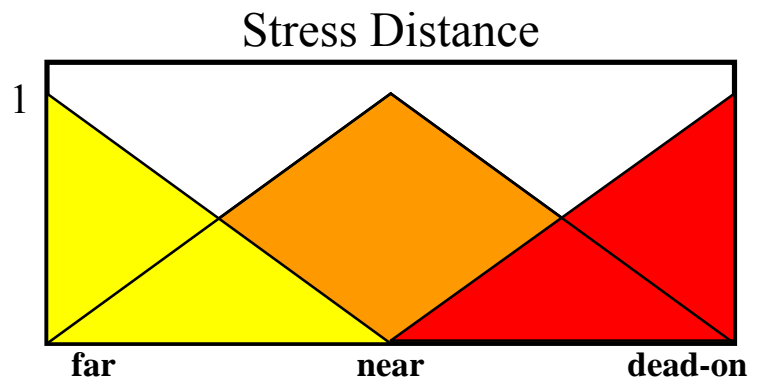

Figure 5-6: Stress Distance Input Membership Functions

\subsubsection{Consequence}

The consequence (output) of the system is the fundamental frequency. The consequence of the fuzzy system has seven linguistic variables. These variables represent the peak of the fundamental frequency. These peaks range from negligible to large. These rule consequences are constructed following the O'Shaughnessy algorithm's peak alterations.

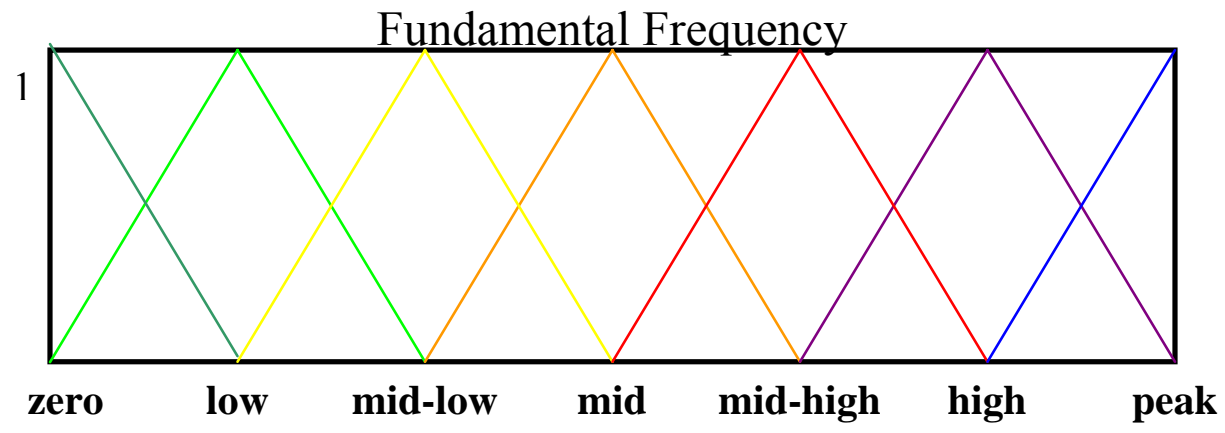

Figure 5-7: Consequence Membership Functions

\subsection{Fuzzy System Rules}

The rules of this system are designed to follow the overall idea of the O'Shaughnessy algorithm. The O'Shaughnessy algorithm is very specific on the shapes of each individual peak. However, these rules create an overall contour of the sentence, 
which follows the O'Shaughnessy algorithm. The rules are divided into three categories: declarative sentences, yes/no questions, and interrogative questions. To simplify each rule set, the rules are divided by location within the sentence. This made it easy to edit, delete, or add rules. Since there are four inputs to the system, there are over 50 rules. This adds the proper detail and improves the flexibility of the fuzzy system.

\subsubsection{Declarative Sentence}

As described in the O'Shaughnessy algorithm, declarative sentences are characterized by the f0 contour falling linearly with a sharp decline at the end of the sentence or phrase. Peaks within the sentence are altered, considering their location in the sentence. Declarative sentences do not have dramatic f0 contour changes inside the middle of the sentence. Therefore, syllables and word importance should produce subtle changes. With declarative sentences, the final fall should be located after the last accented syllable [2].

\section{Beginning of Sentence Rules}

- IF the word is useless AND position in sentence at the beginning THEN output is low.

- IF the word is semi-important AND position in sentence at the beginning THEN output is mid-low.

- IF the word is semi-important AND sentence size is large AND position in sentence at the beginning THEN output is mid.

- IF sentence size is small AND position in sentence at the beginning AND the stress is dead-on THEN output is high.

- IF position in sentence at the beginning AND the stress is dead-on THEN output is peak.

- IF sentence size is small AND position in sentence at the beginning THEN output is mid.

\section{Middle of Sentence Rules}

- IF the word is useless AND position in sentence at the middle THEN output is low.

- IF the word is useless AND sentence size is large AND position in sentence at the middle THEN output is mid-low.

- IF the word is semi-important AND position in sentence at the middle THEN output is mid-low.

- IF the word is semi-important AND sentence size is large AND position in sentence at the middle AND the stress is dead-on THEN output is mid. 
- IF position in sentence at the middle AND the stress is dead-on THEN output is mid-high.

- IF sentence size is medium AND position in sentence at the middle THEN output is mid.

\section{End of Sentence Rules}

- IF sentence is at the end AND the stress is far THEN output is mid-low.

- IF sentence is at the end AND the stress is near THEN output is zero.

- IF sentence size is middle AND sentence at the end THEN output is mid-low.

- IF word is useless AND sentence is at the end THEN output is zero.

\subsubsection{Yes/no Question}

Yes/no questions are questions that can be answered with either a "yes" or a "no". These questions do not begin with "who", "what", "where”, "when”, "why”, or "how". The overall f0 contour of these questions begins with a rise, followed by a flat contour, and then ending with a final sharp rise [2].

\section{Beginning of Sentence Rules}

- IF the word is useless AND position in sentence at the beginning THEN output is mid-low.

- IF the word is semi-important AND position in sentence at the beginning THEN output is mid.

- IF the word is semi-important AND sentence size is large AND position in sentence at the beginning THEN output is mid-low.

- IF position in sentence at the beginning AND the stress is near THEN output is mid-high.

- IF position in sentence at the beginning AND the stress is dead-on THEN output is mid-high.

\section{Middle of Sentence Rules}

- IF the word is useless AND position in sentence at the middle THEN output is mid-low.

- IF the word is useless AND sentence size is large AND position in sentence at the middle THEN output is low.

- IF the word is semi-important AND position in sentence at the middle THEN output is mid-low.

- IF the word is important AND position in sentence at the middle AND the stress is dead-on THEN output is mid-high.

- IF position in sentence at the middle AND the stress is dead-on THEN output is mid. 
- IF sentence size is medium AND position in sentence at the middle THEN output is mid.

- IF sentence size is large AND position in sentence at the middle THEN output is mid-low.

\section{End of Sentence Rules}

- IF sentence is at the end AND the stress is far THEN output is high.

- IF sentence is at the end AND the stress is near THEN output is mid-high.

- IF sentence size is large AND sentence at the end AND the stress is dead-on THEN output is high.

- IF word is useless AND sentence is at the end AND the stress is near THEN output is mid-high.

- IF word is important AND sentence is at the end AND the stress is dead-on THEN output is high.

\subsubsection{Interrogative Question}

Interrogative questions are questions that are answered with more than just a "yes" or "no". These sentences begin with either "who", "what", "where”, “when", "why", or "how". Overall, the f0 contour should initially be high with the final steep fall. The fall of the interrogative question should be much steeper than the fall of the declarative sentence. According to the O'Shaughnessy algorithm, the peaks throughout the sentence should be much higher and the overall contour should be higher than the declarative sentence contour. Syllables and word importance should produce change near accents [2].

\section{Beginning of Sentence Rules}

- IF position in sentence at the beginning THEN output is mid-low.

- IF the word is important AND position in sentence at the beginning THEN output is mid.

- IF the word is semi-important AND sentence size is large AND position in sentence at the beginning THEN output is mid.

- IF sentence size is small AND position in sentence at the beginning AND the stress is dead-on THEN output is mid.

- IF position in sentence at the beginning AND the stress is dead-on THEN output is mid-high.

- IF sentence size is small AND position in sentence at the beginning THEN output is mid-low. 


\section{Middle of Sentence Rules}

- IF the word is useless AND position in sentence at the middle THEN output is mid.

- IF the word is useless AND sentence size is large AND position in sentence at the middle THEN output is mid-low.

- IF the word is semi-important AND sentence size is large AND position in sentence at the middle AND the stress is dead-on THEN output is mid-high.

- IF position in sentence at the middle AND the stress is dead-on THEN output is mid-high.

- IF sentence size is medium AND position in sentence at the middle THEN output is mid.

- IF the word is important AND position in sentence at the middle AND the stress is near THEN output is mid-high.

- IF sentence size is medium AND position in sentence at the middle AND the stress is near THEN output is high.

- IF sentence size is medium AND position in sentence at the middle THEN output is mid-low.

\section{End of Sentence Rules}

- IF sentence is at the end AND the stress is dead-on THEN output is mid-low.

- IF sentence is at the end AND the stress is near THEN output is zero.

- IF sentence size is medium AND sentence at the end THEN output is low.

- IF word is useless AND sentence is at the end THEN output is zero.

\subsection{Fuzzy Output Calculation}

In a fuzzy system, the output is calculated using the rules, the membership

functions, and the inputs. Each input parameter value is calculated using the formulas in Section 5.6. The firing strength of each input is determined by the membership functions. Figure 5-8 shows an example of the Word Importance input with a calculated input of 6 . 


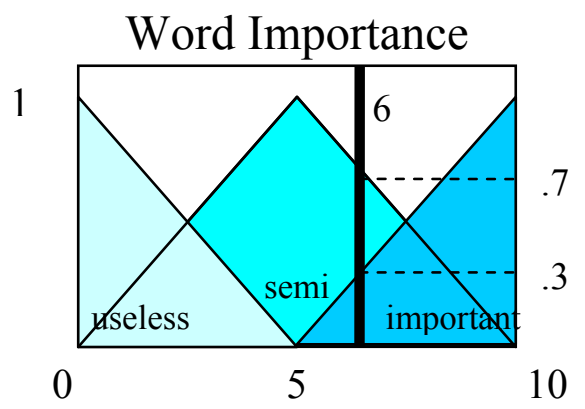

Figure 5-8: Example of Word Importance with Calculated Input of 6

The firing strength of each membership function is shown in Figure 5-8. The membership function "semi" and "important" have a firing strength of .7 and .3, respectively. The "useless" membership function has a firing strength of 0 because the input value does not touch the membership function. The rules of the fuzzy system are used to compute the consequence of the inputs presented to the system. For example, if a rule stated," IF word important is semi-important AND ... ", then for an input value of 6 on input Word Importance, the firing strength onto that rule would be .7. The operators then determine the effects of the input onto the consequence membership function. The $A N D$ operates equals the minimum value and the OR operator equals the maximum value. Figure 5-9 shows an example of how an input to the fuzzy system determines the consequence. For simplicity, there are only two rules and the consequence only has three membership functions. The rules in Figure 5-9 are:

- IF word is importance AND sentence at the end THEN fundamental frequency is high.

- IF word is semi-importance AND sentence at the middle THEN fundamental frequency is middle. 


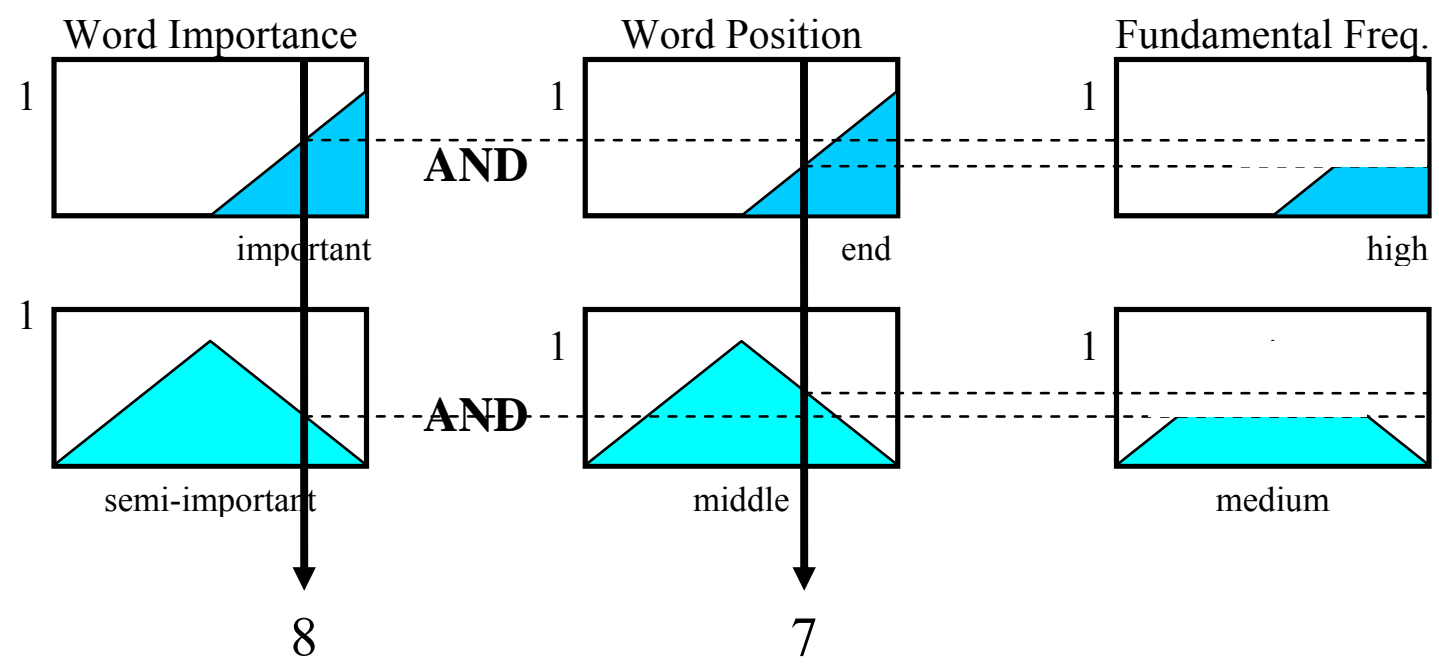

Figure 5-9: Firing Strength of Input onto Rules

The consequences are then combined to produce a final shape. The crisp output of the fuzzy system is calculated by finding the centroid of the final shape.

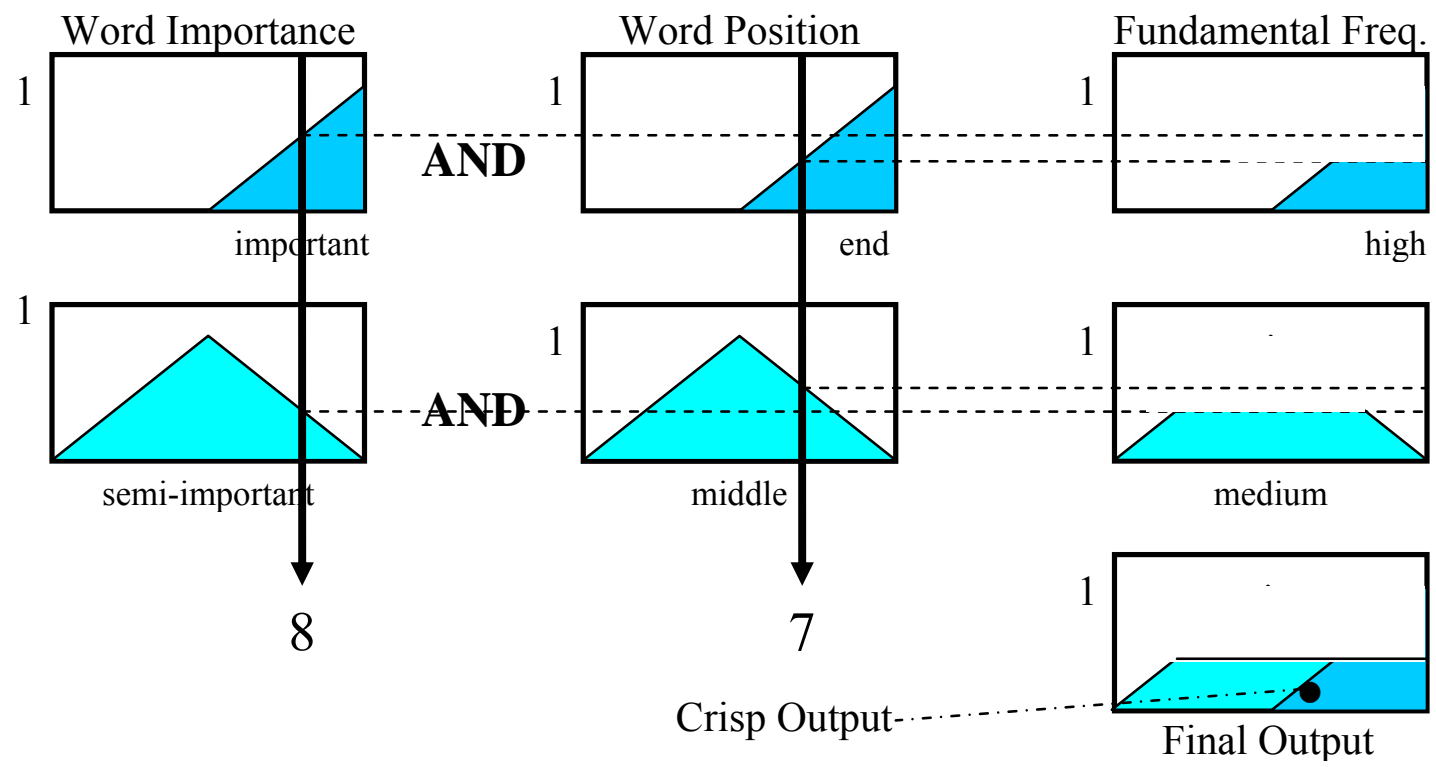

Figure 5-10: Final Shape and Centroid 


\subsection{Final Speech Production}

Once the network is trained and the prosody is calculated, the MBROLA program is used to produce the final speech output. As discussed briefly in Section 3.3, the MBROLA program creates speech using diphone concatenation. This program was developed by the TCTS Lab of the Faculté Polytechnique de Mons in Belgium and was created for use by researchers [15]. The advantage of using the MBROLA program is its ease of use. The only input needed to produce speech is phonemes and durations. Users of MBROLA do not need any knowledge of diphone concatenation algorithms; the program automatically converts the user phonemes to diphones.

The trained hidden and output weights are stored in a MATLAB *.mat file. The TTP conversion is accomplished using the trained weights and formulas. The output is computed using the formulas used to calculate the actual output during training as described in Section 4.2.1.

hidden layer outputs $=1 /\left(1+\mathrm{e}^{-\Sigma(\text { input vector * hidden neurons weights })}\right)$
actual output $=1 /\left(1+\mathrm{e}^{-\Sigma(\text { hidden layer outputs * output neurons weights })}\right)$

The actual output of the system will be a 41 element long vector. The ideal output would be all zeros and a one; however, since the network is not ideal, the maximum of the output needs to be determined. This maximum value of the vector is the output phoneme produced by the network from the text.

When text is entered into the system, the fuzzy controller and the Klatt duration rules compute the prosody of the system. Next, the symbols are converted to the MBROLA symbols. The phonemes, duration, and fundamental frequency values are written to output.pho file. Pho files are the files used by MBROLA to convert phonemes to sounds. The output.pho file is opened and the speech is converted into sound file. See Appendix for more detail on the MBROLA system. 


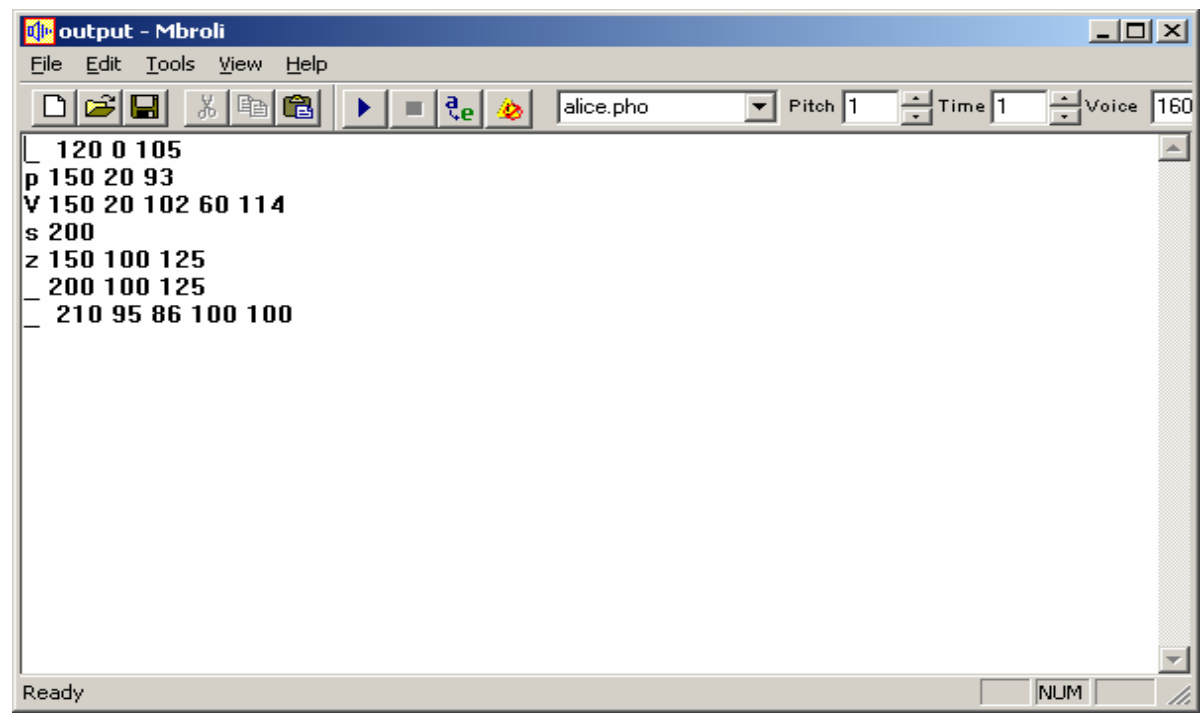

Figure 5-11: MBROLA *.pho file 


\section{Chapter 6: Results}

\subsection{Text-to-Phoneme Results}

Neural networks are used in TTS applications in many different ways. Current research focuses on the use of neural networks to produce prosody. This thesis uses neural networks for TTP mapping. Current neural network TTP mapping techniques yield about an $80 \%$ accuracy [7]. The performance of the neural network algorithm in this system is determined by the amount of accurate phonemes the network identifies. Two types of tests are performed to determine the performance of the algorithm used. The first test calculates the performance using the full training set. The second test uses words that were not in the training set. Performance was measured by the percentage of correct phonetic conversions. Figure 6-1, shows the convergence of the networks. The number of hidden neurons is correlated to the network's convergence. However, after 50 hidden neurons, the network convergance doesn't change much. 


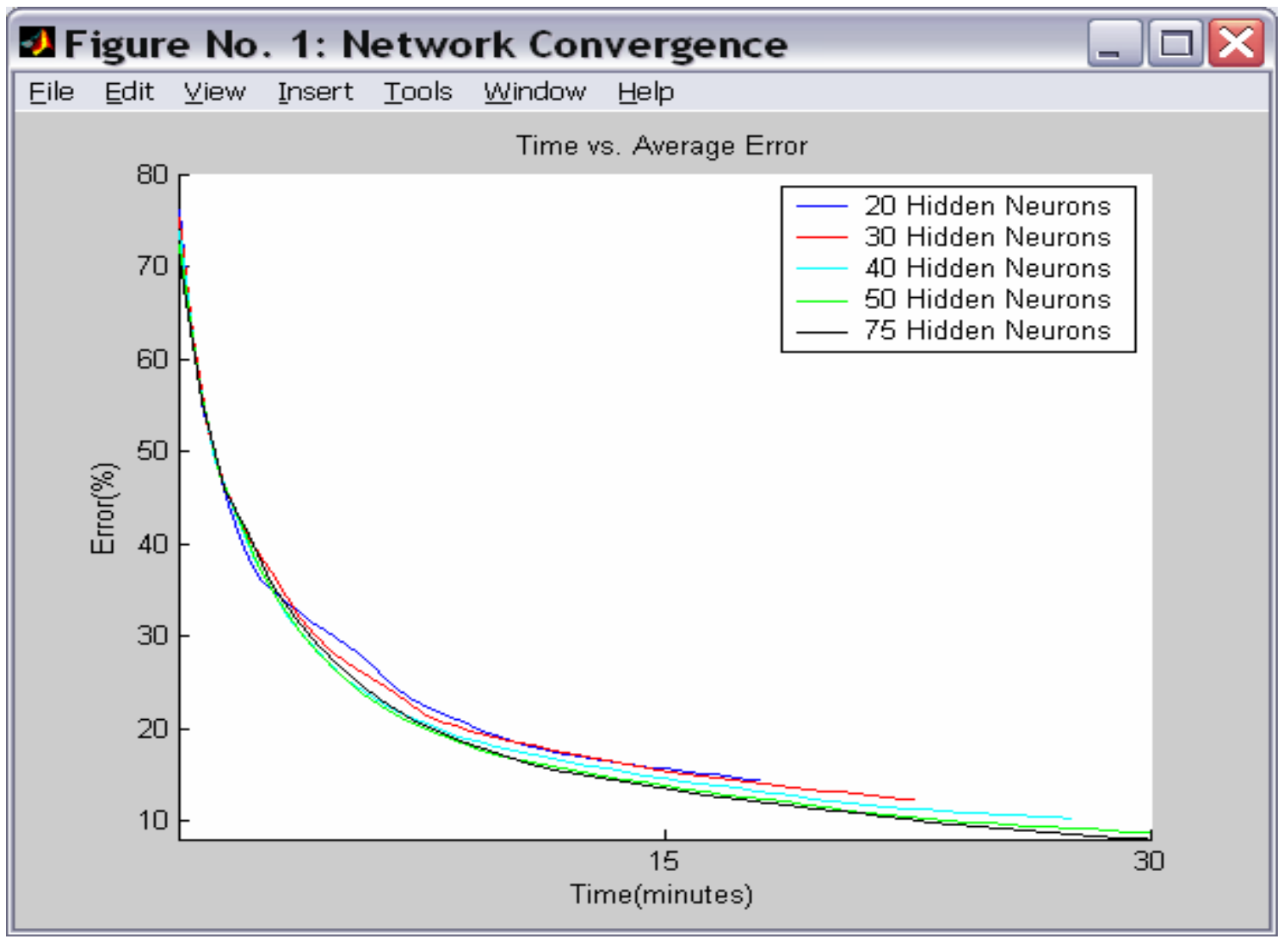

Figure 6-1: Network Convergence

In the first test, network performance is computed by calculating the percentage of correct phonemes determined by the weights on the entire training set. Five different types of trained weights were tested for overall accuracy. The hidden neurons are different for the trained weights sets. The overall performance of the network did change with an increase in the hidden neurons. The maximum accuracy is about $80 \%$. The networks were trained on an Intel Pentium $42.80 \mathrm{GHz}$ computer. Table 6-1 shows the overall accuracy and training results for each network tested.

Table 6-1: First Test Results

\begin{tabular}{|l|l|l|l|l|}
\hline Network & $\begin{array}{l}\text { TTP } \\
\text { Accuracy (\%) }\end{array}$ & $\begin{array}{l}\text { Training Time } \\
(\mathbf{P 4} \text { 2.80GHz) }\end{array}$ & Epochs & Hidden Nodes \\
\hline 1 & 66.93 & $15 \min 10 \mathrm{~s}$ & 75 & 20 \\
\hline 2 & 68.81 & $19 \min 30 \mathrm{~s}$ & 75 & 30 \\
\hline 3 & 72.45 & $22 \min 59 \mathrm{~s}$ & 75 & 40 \\
\hline 4 & 75.77 & $25 \min 13 \mathrm{~s}$ & 75 & 50 \\
\hline 5 & 80.16 & $35 \min 46 \mathrm{~s}$ & 75 & 75 \\
\hline
\end{tabular}


In the second test, network performance is computed by calculating the percentage of correct phonemes calculated on 100 random unknown words (i.e., words that were not in the training set). The same trained weights were tested for overall accuracy. The overall performance, shown in Table 6-2, is about 67\%, which is lower than the performance in the first test. The performance is lower in the second test because of the rule expectations in the English language. For example, the word "vice" is pronounced with a /ie/ phoneme for the "i". But the " $\mathrm{i}$ " in the word "service" is pronounced with a /uh/ phoneme. Consequently, the network does not catch expectations and hence the performance is lower with unknown text.

Table 6-2: Second Test Results

\begin{tabular}{|l|l|}
\hline Network & $\begin{array}{l}\text { TTP } \\
\text { Accuracy (\%) }\end{array}$ \\
\hline 1 & 62.78 \\
\hline 2 & 63.09 \\
\hline 3 & 63.59 \\
\hline 4 & 67.12 \\
\hline 5 & 67.52 \\
\hline
\end{tabular}

Overall, these tests show that the network is about $80 \%$ accurate on the training data. When data not in the training set is introduced, the network performs around $67 \%$ accuracy. When testing the TTS system manually, the text-to-phoneme conversion accuracy is good with some minor faults. The TTP errors of the system might go unnoticed to the user. For example, if the correct (trained) phonetic representation of the word "the" is " $\mid$ th $\backslash \backslash u \backslash$ " and the system produces " $\mid$ th $\backslash$ leel", the user will not notice the incorrect TTP conversion. On the whole, this network is accurate enough to produce the correct text-to-phoneme conversion without many major errors.

\subsection{Fuzzy Fundamental Frequency Results}

Testing the fuzzy system can be complicated. Comparing the sound of the speech produced by the system is somewhat subjective. However, the research objective was to make to the synthesized speech sound more natural using fuzzy logic. Therefore, the fuzzy controller was tested by evaluating the f0 contour produced by the fuzzy system. Two types of tests were used to evaluate the fuzzy system. In the first test, the fo contour 
produced by the system was analyzed to see if it was producing a natural looking f0 contour trend. With speech, only voiced sounds create fundamental frequency.

Therefore, some phonemes will not have fundamental frequency. The fuzzy system was tested by evaluating the three different sentences types. Figure 6-2, 6-3, and 6-4 show the output produced by the system for a declarative sentence, an interrogative question, and a yes/no question, respectively. The sentences are, "My name is Jonathan Williams", "What time is the thesis defense?", and "Is it going to rain at noon?" All three f0 contours produced by the fuzzy system are the accurate f0 trends.

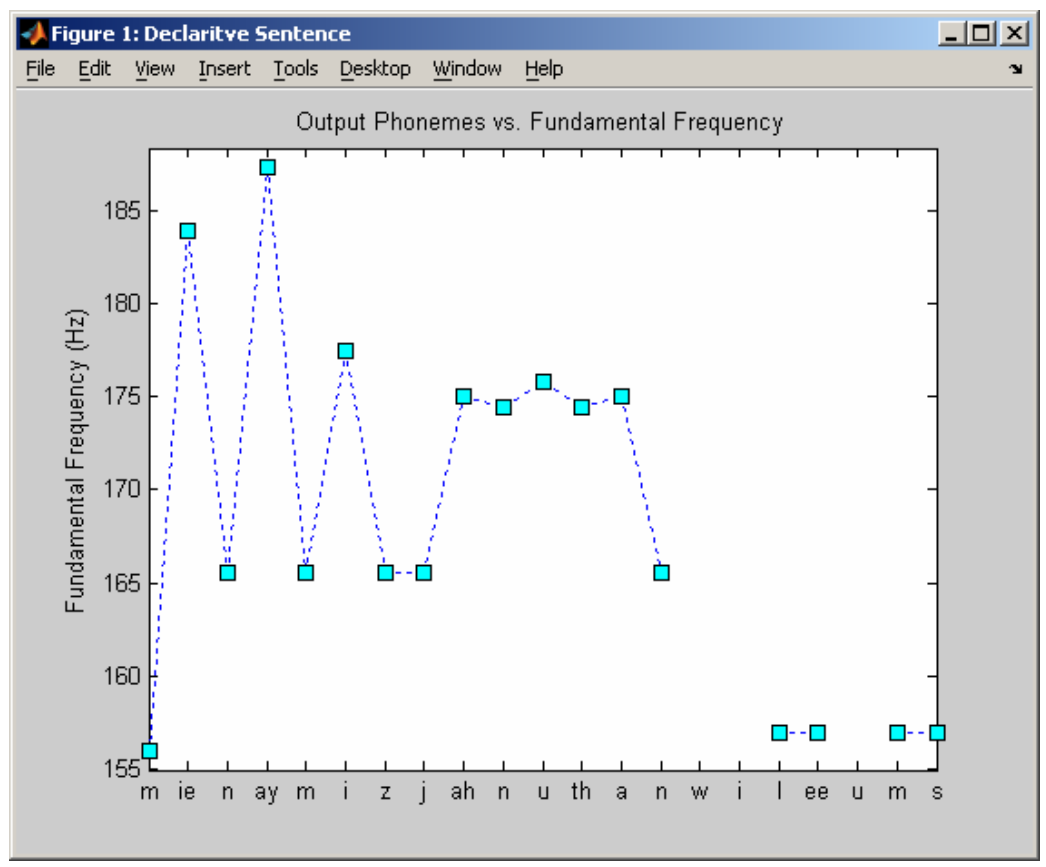

Figure 6-2: Declarative Sentence - "My name is Jonathan Williams" 


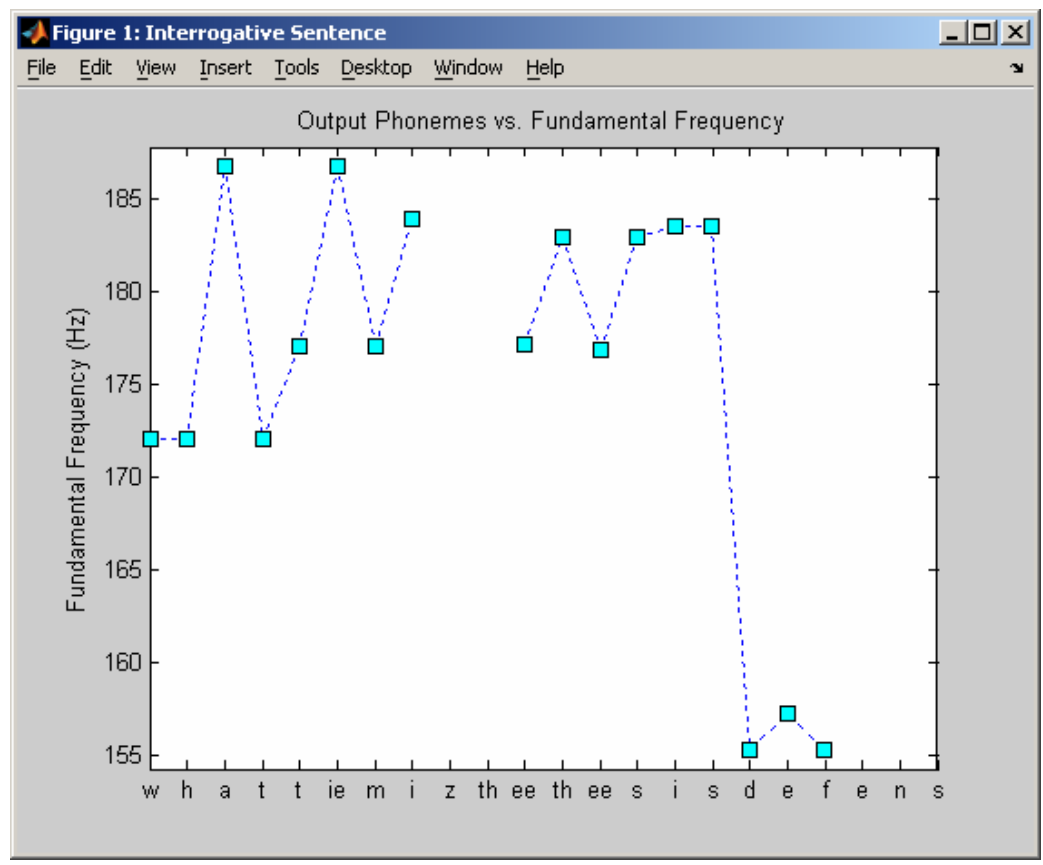

Figure 6-3: Interrogative Question - "What time is the thesis defense?"

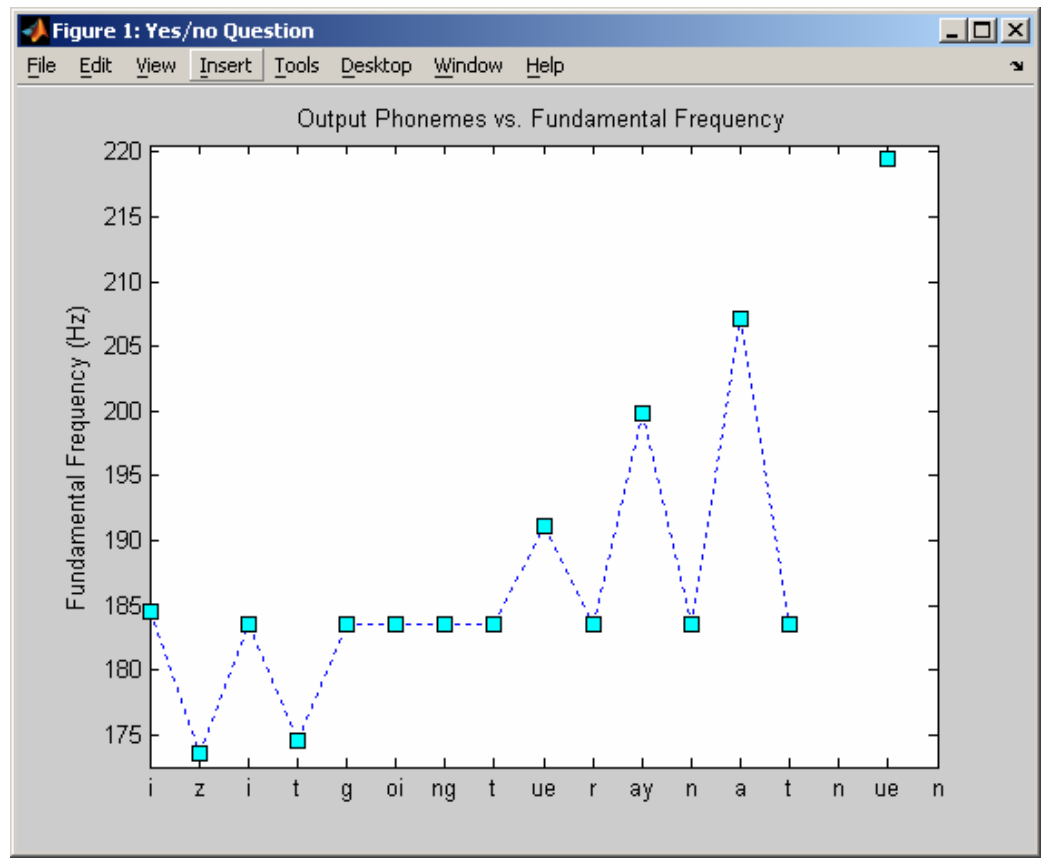

Figure 6-4: Yes/no Question - "Is it going to rain at noon?"

The second test involves viewing the f0 output produced by the fuzzy system with the f0 output of a high quality system. The purpose is to further prove that the fuzzy system's f0 contours are natural looking. It would be impossible to make a comparison 
between both systems, since they are completely different systems. The other f0 contour is the Microsoft Research Speech Technology Asia (MRSA) on-line TTS system, which is one of the best unit-selection based TTS systems [37]. The MRSA system's output f0 contour is determined using the MBROLIGN program. The MBROLIGN program is a tool that aligns phonetic transcripts with a speech signal. MBROLIGN then calculates the $\mathrm{f} 0$ contour of the speech signal. Using the same sentences, three $\mathrm{f} 0$ contours produced by the fuzzy system are graphed with the f0 contours of the MSRA system. The sentence types are declarative sentence, interrogative question, and yes/no question. The following figures show the f0 contour produced by both systems. The sentences are, "My name is Jonathan", "What time is it?" and, "Is it raining today?"

The f0 contour of declarative sentences should be high at the beginning of the sentence and then drop at the end of the sentence. Peaks are on the primary stresses of the stressed syllables. The peak sizes decrease linearly throughout the sentence. For the f0 contours below, both systems produce a downward linear fo contour trend with a drop at the end of the sentence. Both systems also produce peaks on the stressed words within the sentence.

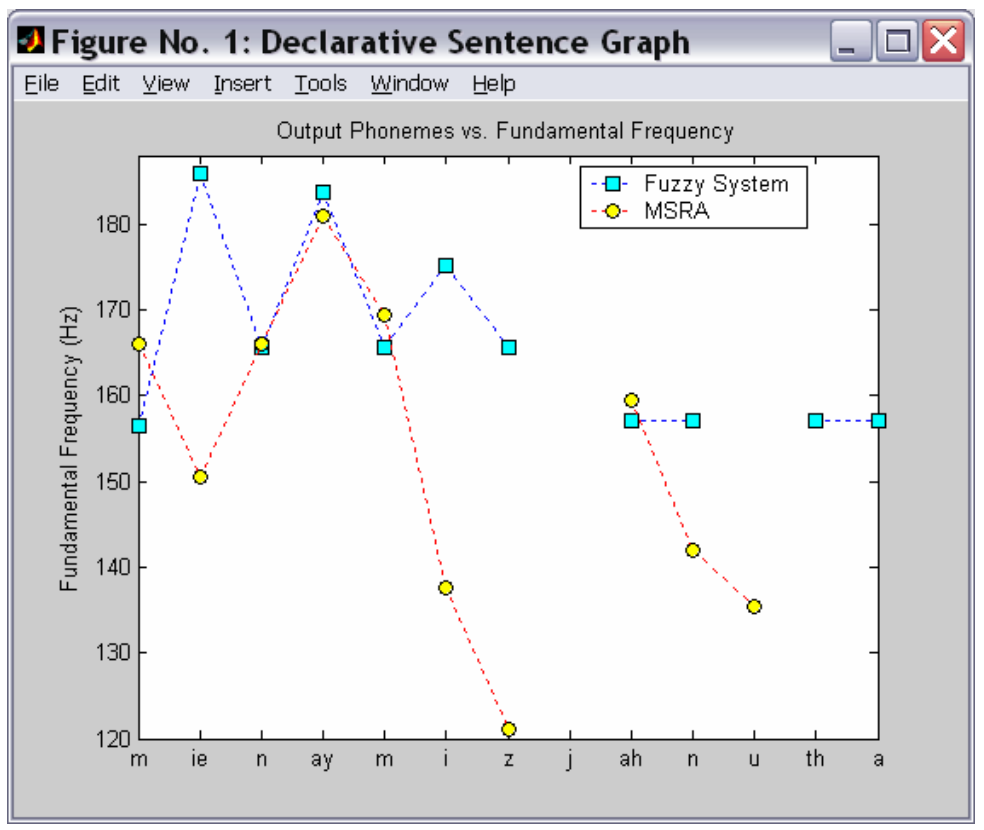

Figure 6-5: F0 Contours for Declarative Sentence, "My name is Jonathan."

The interrogative question $\mathrm{f} 0$ contour is similar to the declarative sentence $\mathrm{f} 0$ contour because both contours fall at the end of the sentence. The interrogative question 
should initially be high with a very sharp drop after the last stress. There should also be peaks around the stresses and the highest peak should be on the first word of the question. These peaks do not fall linearly like the declarative sentence. In the figure below, both contours follow this trend. The fuzzy fo contour peaks on the initial words in the sentence. Then the fuzzy system's f0 contour sharply falls at the end of the sentence.

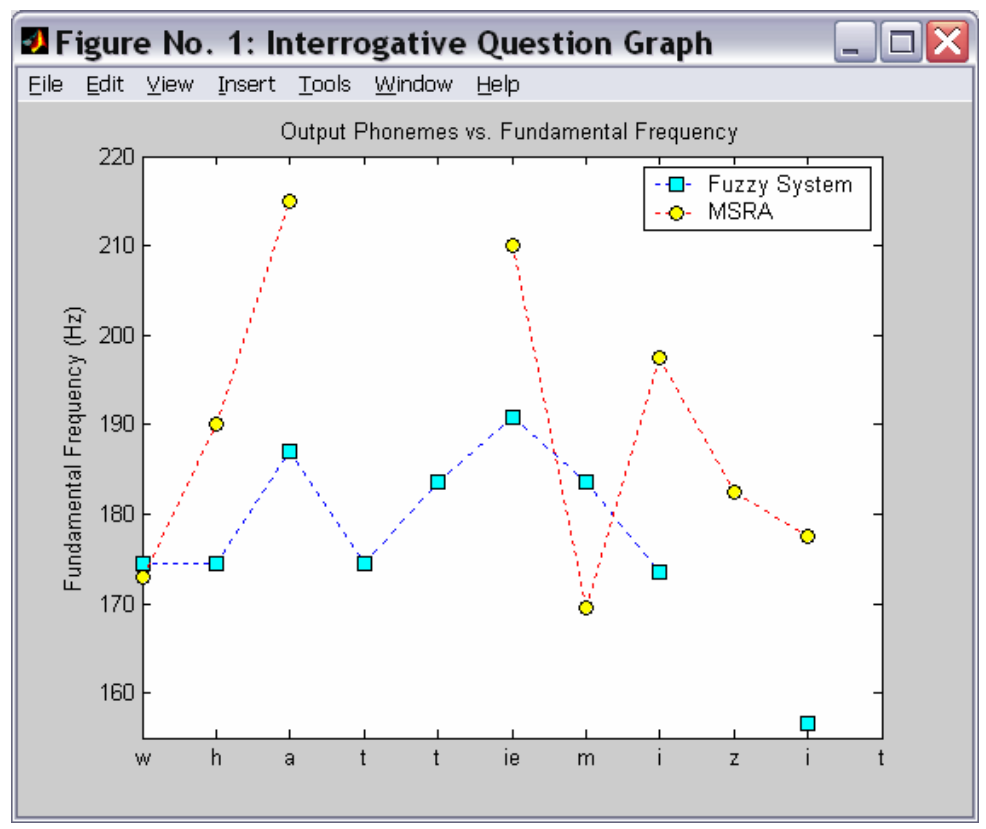

Figure 6-6: F0 Contours for Interrogative Question, "What time is it?"

The yes/no question f0 contour should be relatively low at the beginning with a sharp rise at the end. The peaks throughout the question are smaller compared to the final rise. The graph below shows that the fuzzy f0 contour raises at the end the sentence. The fuzzy controller also has peaks in the middle of the sentence. 


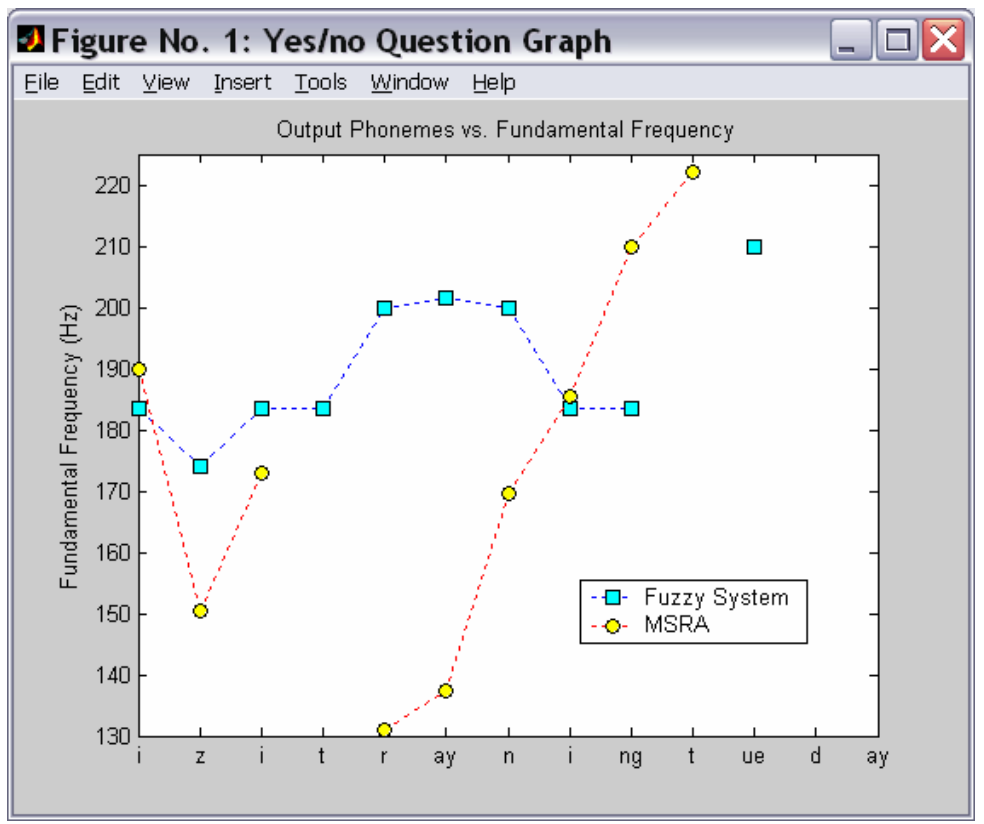

Figure 6-7: F0 Contour Comparison for Yes/no Question, "Is it raining today?" 


\section{Chapter 7: Conclusion}

\subsection{Summary and Conclusion}

The goal was to build a TTS system with fuzzy logic controlling the fundamental frequency of the speech. First, a neural network based on the NetTalk network was trained to convert text into phonemes. The training set contained 1800 words with their phonetic transcription. The Back Propagation algorithm was used to train a three layer network. Then stress was assigned using the Halle and Keyer lexical stress rules. Next, segmental duration was calculated using the Klatt duration rules which were designed to replicate observed segmental durations from a speaker. A fuzzy inference system was built to control the fundamental frequency of the speech output. The inputs to the fuzzy controller were word importance, sentence size, stress location, and sentence position. The output was the fundamental frequency. The system had three sets of rules for three types of sentences: declarative sentence, interrogative question, and yes/no question. Finally, the phonemes and calculated prosodic information was set to the MBROLA program to produce the final speech.

The contribution to this thesis is the idea of using a fuzzy controller to control fundamental frequency. No publications on using fuzzy logic to control fundamental frequency were found. The fuzzy controller generates the expected f0 contour for each of the three sentence types. Thus, the system produces more natural sounding speech. Since evaluating the speech produced by the system is subjective, the f0 contour is compared to a high quality TTS system's f0 contour. In comparison, the fuzzy system produces similar f0 trends compared to the high quality TTS system. However, there are many improvements that can be made to the fuzzy fundamental frequency controller.

Overall, the final speech produced by the TTS system sounds more natural. The speech is very understandable and the user can hear the intonation. The purpose of this thesis was to explore the possibilities of using fuzzy logic for automatic prosodic control. This thesis proves that fuzzy logic can a make low memory method of speech synthesis sound more natural. Currently, unit selection is the best sounding speech synthesis method. However, the speech databases must be segmented and the speech databases 
sizes are measured in gigabytes. Compared to unit selection-based synthesis, the diphone database does not need to be segmented and the speech database requires a small amount of memory. Using the MBROLA diphone concatenation program, the total program size is under $8 \mathrm{MB}$. The TTS system itself is less than a megabyte in size and the diphone database is only $6.75 \mathrm{MB}$. With further research, fuzzy logic controlled speech could be the preferred speech synthesis method for our shrinking electronics.

\subsection{Future Work}

The neural network text to phoneme conversion accuracy needs to improve. Currently, the Back Propagation algorithm yields the best correctness. Different methods like Bi-Direction Recurrent Networks (BRNN) and Self-Organizing Maps (SOM) produce about 70\% TTP conversion accuracy [7]. Performance can be improved in many ways. One way to improve network performance is to increase the size of the training set. The results showed that the network produces $80 \%$ accuracy on known text and about $70 \%$ accuracy of unknown text. Therefore, minimizing the amount of unknown text during training would yield better results. However, the algorithm would still produce errors. Improvements to the Back Propagation method or other machine learning methods need to be further researched in order for machine learning methods to be comparable to the current technologies. Also, research into error correction after training would be very beneficial to the acceptance of the machine learning method.

Improvements to certain components of the TTS system would produce more natural-sounding speech. The stress assignment algorithm can be greatly improved. The current stress assignment method does not assign stress with complete accuracy. Improvements to the system stress algorithm would improve the prosody and naturalness

of the speech output. A part-of-speech parser also needs to be added to the system. The current TTS system only handles two types of words: function and content. Assigning word importance based on the part-of-speech would also improve the speech output.

Although the fuzzy controller works adequately, the fuzzy system needs improvements. One improvement can be on the actual rules of the fuzzy system. Decreasing the amount of rules could improve the speed, since the system computation requirements would decrease. The system currently has over 50 rules. Another 
improvement can be on the composition of the rules. There needs to be research into designing expert rules for a fuzzy system controlling prosody. If more research went into creating expert fuzzy prosodic rules, a fuzzy system could control fundamental frequency and other aspects of prosody, like segmental duration and stress assignment. The membership functions may not be optimized in the fuzzy system. Research into different types of membership function could also produce more natural sounding speech. The use of different types of membership functions or the use of ANFIS could improve the fuzzy system. 


\section{References}

1. Adamson, M. J., and Robert I. Damper. "A Recurrent Network that Learns to Pronounce English Text", ICSLP-1996, (1996), 1704-1707.

2. Allen, John, Hunnicutt, Sharon, and Dennis Klatt. From Text To Speech, The MITTALK System. Cambridge: Cambridge University Press, 1987.

3. Anderson, Mark, and Janet Pierrehumbert. "Synthesis by Rule of English Intonation", Proceedings of the International Conference on Acoustic, Speech and Signal Processing 84, (1984), 281-284.

4. Arcieniegas, Fabio and Mark J. Embrechts. "Text-To-Speech Conversion with Staged Neural Networks", Intelligent Engineering Systems through Artificial Neural Networks: Smart Engineering System Design: Vol. 10, C. H. Dagli et al., eds., ASME Press, (2000), 733-738.

5. “AT\&T Natural Voices - Home Page.” 2002. http://www.naturalvoices.att.com/ (4/13/2005).

6. Beatrice, Enikö, Astola, Jaakko, and Jukka Saarinen. "Recurrent Neural Network with Both Side Context Dependence for Text-To-Phoneme Mapping ", Proceedings of the IEEE First International Symposium on Control, Communications, and Signal Processing, ISCCSP 2004, Hammamet, Tunisia, March, (2004).

7. Bilcu, Beatrice, Salmela, Janne, and Jaarinen Saarinen. "Application of the Neural Networks for Text-to-phoneme Mapping”, Proceedings of XI European Signal and Image Processing Conference, EUSIPCO 2002, Toulouse, France, September, (2002), 892-896.

8. Black, A., and P. Taylor. The Festival Speech Synthesis System: system documentation, Human Communications Research Centre, University of Edinburgh, Scotland, January 1997.

9. Brule, James F. "Fuzzy Systems - A Tutorial.” 1999. www.austinlinks.com/Fuzzy/tutorial.html/ (6/1/2005).

10. Buhmann, Jeska, Vereecken, Halewijn, Fackrell, Justin, Martens, Jean-Pierre and Bert van Coile. "Data driven intonation modelling of 6 languages", ICSLP-2000, vol.3, 179-182 (2000).

11. Campbell, W.N. "Syllable-based segmental duration", Talking Machines:

Theories, Models and Designs, Amsterdam: Elsevier Science Publishers, 211224 
12. Damper, Robert I., and Y. Marchand. "Improving Pronunciation by Analogy for Text-to-Speech Applications", SSW3-1998, (1998), 65-70.

13. Dedina, M. J., and H. C. Nusbaum. "PRONOUNCE: A program for pronunciation by analogy", Computer Speech and Language, 5(1):55-64, (1991).

14. Dusterhoff, Kurt E., Black, Alan W. and Paul A. Taylor. "Using Decision Trees within the Tilt Intonation Model to Predict F0 Contours", Eurospeech 99, (1999).

15. Dutoit, T., and H. Leich. "MBR-PSOLA: Text-To-Speech Synthesis Based on an MBE Re-Synthesis of the Segments Database", Speech Communication, Elsevier Publisher, vol. 13, no. 3-4 (1993).

16. Dutoit, T., An Introduction to Text-To-Speech Synthesis, Kluwer Academic Publishers, 1996.

17. Fujsaki, H. "The Role of Quantitative Modeling in the Study of Intonation", Proceedings of the International Symposium on Japanese Prosody, (1992), 163-174

18. Gubbins, P.R., Curtis, K.M., and J.D. Burniston. “A Hybrid Neural Network/Rule Based Architecture Used as a Text to Phoneme Transcriber", Proc. International Symposium on Speech, Image Processing and Neural Networks (ISSIPNN’94), Hong Kong, April, (1994), 113-116.

19. Hemming, Cecilia. Department of Languages, University College of Skövde Swedish National Graduate School of Language Technology. "Using Neural Networks in Linguistic Resources" 2002. http://www.hemming.se/gslt/LingRes/NeuralNetworks.htm/ (4/19/2005).

20. Klatt, Dennis. "Linguist Use of Segmental Duration in English: Acoustic and Perceptual Evidence", Journal of the Acoustical Society of America, 59, vol. 3, 1208-1221, (1976).

21. Klatt, Dennis. "Review of Text-to-Speech Conversion for English", Journal of the Acoustical Society of America, 82, vol. 3, 737-793, (1987).

22. Klatt, Dennis. "The Klattalk Text-to-Speech Conversion System", Proceedings of ICASSP 82, (1982), 1589-1592.

23. Mattingly, I. G. "Speech Synthesis for Phonetic and Phonological Models", T.A. Sebeok (Ed.) Current Trends in Linguistics, vol 12, Linguistics and Adjacent Arts and Sciences, vol. 4, Mouton: The Hague. 2451-2487, (1974). 
24. McCulloch, N., Bedworth, M., and J. Bridle. "NETspeak - A Reimplementation of NETtalk”, Computer Speech and Language, vol. 2, 284301, (1987).

25. Nakajima, S. and H. Hamada. "Automatic Generation of Synthesis Units Based on Context oriented Clustering," Proc. ICASSP-88, (1987).

26. Öhman, S. "Word and Sentence Intonation: a Quantitative Model", K.T.H. Quarterly Progress Report, vol. 2, 25-54, (1969).

27. Olive, J. P. and N. Spickenagel. "Speech Resynthesis from Phoneme-related Parameters," JASA, vol. 59, no. 4, Apr 1976, 993-996.

28. O'Saughnessy, D. Speech Communication - Human and Machine, Reading, PA: Addison-Wesley, 1987.

29. "Phoneme." Encyclopedia on Labor Law Talk [online encyclopedia] (Jelsoft Enterprises Ltd. 2000); available from http:/encyclopedia.laborlawtalk.com/ (7/3/2005).

30. Pierrehumbert, Janet. 'Synthesizing Intonation”, Journal of the Acoustical Society of America, vol. 70, no. 4, 1981, 985-995.

31. Taylor, P. "The Tilt Intonation Model”, In R. Mannell and J. Robert-Ribes, eds, Proc. ICSLP 98, vol. 4, (1998), 1383-1386.

32. Riley, M.D. "Tree-based Modeling for Speech Synthesis", Talking Machines: Theories, Models, and Designs, G. Baily and C. Benoit, eds., North Holland, 265-273, (1992).

33. Schroeder, M. "A Brief History of Synthetic Speech”. Speech Communication, vol. 13, 231-237, (1993).

34. Scordilis, M. and J. Gowdy. "Neural Network Based Generation of Fundamental Frequency Contours", IEEE International Conference on Acoustics, Speech, and Signal Processing, (1989), 219-222.

35. Sejnowski, T. J., and C. R. Rosenberg. "Parallel Network that Learn to Pronounce English Text," Complex Systems, vol. 1, no. 1, 145-168, (1987).

36. "Speech Synthesis." Wikipedia, the Free Encyclopedia [encyclopedia online] (Wikimedia Foundation, Inc.); available from http://en.wikipedia_.org/wiki/.

37. "Speech Technology." Microsoft Research Speech Technology (Asia). 2005. http://www.research.microsoft.com/speech/ (7/19/2005). 
38. Stacey, Deborah and Steven Kramer. "Artificial Neural Networks: From McCulloch Pitts to Back Propagation.” Jan. 1999.

http://hebb.cis.uoguelph.ca/ skremer/Teaching/27642/BP/BP.html/ $(6 / 2 / 2005)$.

39. Sullivan, K.P., and R.I., Damper. "Novel Word Pronunciation: A Crosslallgtlage Study", Speech Communications, vol. 13, 441-452, (1993).

40. Tangsangiumvisai, Nisachon. Department of Electrical Engineering, Chulalongkorn University. "Study of LPC Speech" 2004.

http://www.student.chula.ac.th/ 47704705/index.html/ (5/16/05).

41. “Timeline: Digital Computing, DECTalk” Microsoft Research. 21 April 1998. http://research.microsoft.com/ gbell/Digital/timeline/1983-5.htm (7/15/05).

42. Zadeh, Lofti. "Fuzzy Sets.” Journal of Information and Control 8, 338-353, (1965). 


\section{Appendix A - Phoneme List}

\begin{tabular}{|c|c|c|}
\hline Network & MBROLA & Sound \\
\hline $\mathrm{a}$ & $\overline{\{}$ & apple \\
\hline ah & $A$ & Arthur \\
\hline aw & O & all \\
\hline ay & $\mathrm{El}$ & able \\
\hline$b$ & $b$ & cob \\
\hline ch & tS & notch \\
\hline$d$ & $d$ & nod \\
\hline e & $E$ & else \\
\hline ee & i & even \\
\hline$f$ & $f$ & for \\
\hline g & g & jog \\
\hline $\mathrm{h}$ & $\mathrm{h}$ & harm \\
\hline i & 1 & illness \\
\hline ie & $\mathrm{Al}$ & īsland \\
\hline j & Z & garage \\
\hline$k$ & $\mathrm{k}$ & rock \\
\hline I & 1 & doll \\
\hline $\mathrm{m}$ & $\mathrm{m}$ & palm \\
\hline$n$ & $\mathrm{n}$ & johñ \\
\hline $\mathrm{ng}$ & $\mathrm{N}$ & bong \\
\hline oh & $@ \cup$ & over \\
\hline oi & OI & oyster \\
\hline oo & $U$ & good \\
\hline ow & $\mathrm{aU}$ & out \\
\hline $\mathrm{p}$ & $p$ & drop \\
\hline$r$ & $r$ & star \\
\hline $\mathrm{s}$ & s & boss \\
\hline sh & $\mathrm{S}$ & wash \\
\hline $\mathrm{t}$ & $\mathrm{t}$ & plot \\
\hline th & $\mathrm{T}$ & cloth \\
\hline $\mathrm{u}$ & @ & about \\
\hline ue & $\mathrm{u}$ & oodles \\
\hline uh & $\mathrm{V}$ & $\overline{\text { nut }}$ \\
\hline ur & $r=$ & her \\
\hline $\mathrm{v}$ & $v$ & salve \\
\hline w & W & show \\
\hline $\mathrm{xh}$ & $\mathrm{D}$ & clothe \\
\hline y & j & yacht \\
\hline$z$ & z & was \\
\hline zh & $\mathrm{s}$ & seižzure \\
\hline
\end{tabular}




\section{Appendix B - Phoneme Inherit Duration}

\begin{tabular}{|c|c|c|}
\hline Phoneme & Minimum(ms) & (ms) \\
\hline a & 80 & 230 \\
\hline ah & 100 & 240 \\
\hline aw & 100 & 240 \\
\hline ay & 70 & 150 \\
\hline e & 100 & 190 \\
\hline ee & 55 & 155 \\
\hline i & 40 & 135 \\
\hline ie & 150 & 250 \\
\hline oh & 80 & 220 \\
\hline oi & 150 & 280 \\
\hline 00 & 60 & 160 \\
\hline ow & 100 & 260 \\
\hline $\mathrm{u}$ & 60 & 120 \\
\hline ue & 70 & 210 \\
\hline uh & 60 & 140 \\
\hline ur & 80 & 180 \\
\hline $\mathrm{h}$ & 20 & 80 \\
\hline I & 40 & 80 \\
\hline$r$ & 30 & 80 \\
\hline w & 60 & 80 \\
\hline y & 40 & 80 \\
\hline $\mathrm{m}$ & 60 & 70 \\
\hline $\mathrm{n}$ & 50 & 60 \\
\hline $\mathrm{ng}$ & 60 & 95 \\
\hline$f$ & 80 & 100 \\
\hline $\mathrm{s}$ & 60 & 105 \\
\hline sh & 80 & 105 \\
\hline th & 60 & 90 \\
\hline v & 40 & 60 \\
\hline $\mathrm{xh}$ & 30 & 50 \\
\hline z & 40 & 75 \\
\hline zh & 40 & 70 \\
\hline$b$ & 60 & 85 \\
\hline$d$ & 50 & 75 \\
\hline g & 60 & 80 \\
\hline k & 60 & 80 \\
\hline $\mathrm{p}$ & 50 & 90 \\
\hline $\mathrm{t}$ & 50 & 75 \\
\hline ch & 50 & 70 \\
\hline j & 50 & 70 \\
\hline$\%$ & 25 & 25 \\
\hline
\end{tabular}




\section{Appendix C - Training Set}

\begin{tabular}{|c|c|c|c|c|c|}
\hline a & lu/ & guts & /guts/ & Renee & /rennay/ \\
\hline abbey & labbee/ & habits & /habits/ & reply & /riplie/ \\
\hline able & laybul// & hacker & /hackur/ & report & /ripawrt/ \\
\hline about & /ubowt/ & had & /had/ & require & /rikwier/ \\
\hline above & /ubuhv/ & hags & /hagz/ & rest & /rest/ \\
\hline abs & labzl & hail & /hayl/ & result & /rizuhlt/ \\
\hline action & /akshun/ & hair & /her/ & retch & /rech/ \\
\hline add & lad/ & half & /haf/ & return & /riturn/ \\
\hline adjoin & /adjoin/ & hall & /hawl/ & rich & /rich/ \\
\hline advance & /udvans/ & hallo & /hawloh/ & riches & /richiz/ \\
\hline affix & /ufiks/ & halo & /hayloh/ & ridden & /ridun/ \\
\hline afraid & /ufrayd/ & halt & /halt/ & ride & /ried/ \\
\hline after & /aftur/ & halve & /hav/ & right & /riet/ \\
\hline again & /ugen/ & hamper & /hampur/ & ring & /ring/ \\
\hline age & /ayj/ & hand & /hand/ & rise & /riez/ \\
\hline agree & /ugree/ & handle & /handul/ & river & /rivur/ \\
\hline ahoy & /uhoi/ & handy & /handee/ & road & /rohd/ \\
\hline air & ler/ & hang & /hang/ & rock & /rawk/ \\
\hline airplane & /erplayn/ & happen & /hapun/ & roll & /rohl/ \\
\hline airway & lerway/ & happy & /hapee/ & room & /ruem/ \\
\hline all & /awl/ & hard & /hahrd/ & round & /rownd/ \\
\hline alley & /alee/ & hardly & /hawrdlee/ & row & /roh/ \\
\hline alloy & /owlloi/ & hardy & /hawrdee/ & royal & /roiyul/ \\
\hline along & /ulawng/ & hare & /her/ & ruby & /ruebee/ \\
\hline already & /awlredi/ & has & /haz/ & ruches & /roochiz/ \\
\hline also & /awlsoh/ & hasty & /haystee/ & rule & /ruel/ \\
\hline although & /awlthoh/ & hat & /hat/ & rummy & /ruhmee/ \\
\hline always & /awlwayz/ & hatch & /hach/ & run & /ruhn/ \\
\hline am & lam/ & have & /hav/ & rush & /ruhsh/ \\
\hline amount & /umownt/ & he & /hee/ & rush & /rush/ \\
\hline and & land/ & head & /hed/ & rushes & /ruhshiz/ \\
\hline anger & /anggur/ & heal & /heel/ & rusty & /ruhstee/ \\
\hline Anglo & /angloh/ & health & /helxh/ & sad & /sad/ \\
\hline angry & langgree/ & health & /helth/ & safety & /sayftee/ \\
\hline animal & /anumul/ & heap & /heep/ & said & /sed/ \\
\hline annex & laneks/ & hear & /hir/ & sail & /sayl/ \\
\hline annoy & /unnoi/ & heard & /hurd/ & salt & /sawlt/ \\
\hline anoint & /unoint/ & heart & /hahrt/ & same & /saym/ \\
\hline anomie & /unahmee/ & heat & /heet/ & sandy & /sandee/ \\
\hline anomy & /unahmee/ & heater & /heetur/ & sashes & /sashizl \\
\hline another & /unuhthur/ & heaven & /hevun/ & sat & /sat/ \\
\hline anoxia & /anoksu/ & heavy & /hevee/ & Saturday & /Saturday/ \\
\hline answer & /ansur/ & hedge & /hej/ & save & /sayv/ \\
\hline antics & /antiks/ & height & /hiet/ & saw & /saw/ \\
\hline any & /eni/ & held & /held/ & say & /say/ \\
\hline apex & laypeks/ & hello & /heloh/ & scene & /seen/ \\
\hline
\end{tabular}




\begin{tabular}{|c|c|c|c|c|c|}
\hline appear & /upir/ & help & /help/ & school & /skuel/ \\
\hline apple & lapul/ & hem & /hem/ & sea & /see/ \\
\hline April & /ayprul/ & her & /hur/ & seat & /seet/ \\
\hline arch & /arch/ & herbs & /hurbz/ & second & /sekund/ \\
\hline are & lahr/ & here & /hir/ & see & /see/ \\
\hline arm & /ahrm/ & heresy & /herusee/ & seed & /seed/ \\
\hline army & /ahrmee/ & hero & /heeroh/ & seem & /seem/ \\
\hline around & /urownd/ & hers & /hurzl & seen & /seen/ \\
\hline array & larray/ & hey & /hay/ & self & /self/ \\
\hline arrive & /uriev/ & hey & /hayl & sell & /sel/ \\
\hline art & lart/ & hiccup & /hikup/ & sense & /sens/ \\
\hline article & /ahrtukul/ & hiding & /hieding/ & sent & /sent/ \\
\hline as & lazl & high & /hie/ & separate & /seprit/ \\
\hline ash & lash/ & hijack & /hiejak/ & September & /septembur/ \\
\hline ashes & /ashiz/ & hike & /hiek/ & serve & /surv/ \\
\hline ask & lask/ & hikes & /hiekz/ & service & /survis/ \\
\hline astray & /ustray/ & hill & /hil/ & set & /set/ \\
\hline at & lat/ & him & /him/ & settle & /setul/ \\
\hline attach & /uttach/ & hinder & /hindur/ & seven & /sevun/ \\
\hline attept & /utempt/ & hint & /hint/ & several & /sevrul/ \\
\hline August & /Awgust/ & hip & /hip/ & shabby & /shabbee/ \\
\hline aunt & lant/ & hire & /hieur/ & shade & /shayd/ \\
\hline avoid & /uvoid/ & his & /hiz/ & shake & /shayk/ \\
\hline away & /uway/ & history & /histree/ & shall & /shal/ \\
\hline$a x$ & laks/ & hit & /hit/ & sham & /sham/ \\
\hline axis & /aksis/ & hoax & /hoaks/ & shame & /shaym/ \\
\hline baby & /baybee/ & hobble & /hawbul/ & shape & /shayp/ \\
\hline back & /bak/ & hobby & /hahbee/ & share & /sher/ \\
\hline bad & /bad/ & hold & /hohld/ & shark & /shahrk/ \\
\hline baq & $/ \mathrm{baq} /$ & hole & /hohl/ & sharp & /shahrp/ \\
\hline balk & /bawlk/ & holy & /hohlee/ & shave & /shayv/ \\
\hline ball & /bawl/ & home & /hohm/ & she & /shee/ \\
\hline balmy & /bahlmee/ & homely & /hohmee/ & shed & /shed/ \\
\hline bangs & /bangz/ & homy & /hohmee/ & sheep & /sheep/ \\
\hline bank & /bangk/ & honey & /huhnee/ & sheer & /shirl \\
\hline banker & /bangkur/ & hoof & /hoof/ & sheet & /sheet/ \\
\hline barmy & /bahrmee/ & hook & /hook/ & shelf & /shelf/ \\
\hline bash & /bash/ & hooks & /hooks/ & shell & /shel/ \\
\hline basket & /baskut/ & hoops & /huepz/ & shield & /sheeld/ \\
\hline batch & /bach/ & hope & /hohp/ & shift & /shift/ \\
\hline bath & /bath/ & horrid & /hawrid/ & shine & /shien/ \\
\hline baths & /bathz/ & horse & /hawrs/ & ship & /ship/ \\
\hline battle & /batul/ & hot & /haht/ & ships & /shipz/ \\
\hline batty & /batee/ & hotty & /hawtee/ & shirk & /shirk/ \\
\hline baulk & /bowlk/ & hour & lowr/ & shirt & /shurt/ \\
\hline bawdy & /bawdee/ & hours & lowrzl & shit & /shit/ \\
\hline bay & /bay/ & house & /hows/ & shock & /shahk/ \\
\hline bay & /bay/ & how & /how/ & shoe & /shue/ \\
\hline be & /bee/ & however & /howevur/ & shoot & /shuet/ \\
\hline bean & /been/ & hugs & /huhgz/ & shop & /shahp/ \\
\hline
\end{tabular}




\begin{tabular}{|c|c|c|c|c|c|}
\hline beauty & /beeuetee/ & hulk & /hohlk/ & shore & /shawr/ \\
\hline became & /bikaym/ & humid & /huemid/ & short & /shawrt/ \\
\hline because & /bikawz/ & humor & /huemawr/ & shot & /shaht/ \\
\hline become & /bikuhm/ & hump & /huhmp/ & should & /shood/ \\
\hline bed & /bed/ & hums & /huhmzl & shoulder & /shohldur/ \\
\hline beech & /beech/ & hunch & /huhnch/ & shout & /showt/ \\
\hline been & /bin/ & hungry & /hunggree/ & shove & /shuhv/ \\
\hline before & /bifawr/ & hunt & /huhnt/ & show & /shoh/ \\
\hline began & /bigan/ & hurry & /huree/ & shower & /showur/ \\
\hline begin & /bigin/ & hurt & /hurt/ & shown & /shohn/ \\
\hline behind & /bihiend/ & husband & /huhzbund/ & shred & /shred/ \\
\hline being & /beeing/ & I & lie/ & shrill & /shril/ \\
\hline believe & /bileev/ & ice & /ies/ & shrine & /shrien/ \\
\hline bell & /bel/ & idea & /iediu/ & shrink & /shrink/ \\
\hline belong & /bilawng/ & if & /if/ & shrug & /shruhg/ \\
\hline below & /biloh/ & iffy & /iffeel & sick & /sik/ \\
\hline bends & /bendz/ & ilk & /ilk/ & side & /sied/ \\
\hline berth & /berth/ & ill & /il// & sight & /siet/ \\
\hline beside & /busied/ & important & /impawrtunt/ & sights & /siets/ \\
\hline best & /best/ & in & /in/ & sign & /sien/ \\
\hline betray & /beetray/ & inch & /inch/ & silk & /silk/ \\
\hline better & /betur/ & inches & /inchiz/ & silver & /silvur/ \\
\hline between & /bitween/ & include & /inklued/ & simple & /simpul/ \\
\hline beyond & /biyahnd/ & increase & /inkrees/ & since & /sins/ \\
\hline bicycle & /biesikul/ & indeed & /indeed/ & sing & /sing/ \\
\hline big & /big/ & industry & /industree/ & single & /singgul/ \\
\hline bilk & /bilk/ & inside & /insied/ & sister & /sistur/ \\
\hline birch & /burch/ & instead & /insted/ & six & /siks/ \\
\hline bird & /burd/ & into & /intue/ & sixty & /siktee/ \\
\hline birth & /berth/ & iron & /ieurn/ & size & /siez/ \\
\hline black & /blak/ & is & lizl & skulk & /skuhlk/ \\
\hline bleach & /bleech/ & it & /it/ & sleep & /sleep/ \\
\hline blood & /bluhd/ & itch & /ich/ & slept & /slept/ \\
\hline bloody & /bluhdee/ & its & /its/ & slow & /sloh/ \\
\hline blow & /bloh/ & jab & /jab/ & small & /smawl/ \\
\hline blue & /blue/ & jabber & /jabur/ & smell & /smel/ \\
\hline blush & /bluhsh/ & jack & ljakl & smoke & /smohk/ \\
\hline board & /bawrd/ & jacket & /jakit/ & snow & /snoh/ \\
\hline boat & /boht/ & jade & /jayd/ & so & /soh/ \\
\hline body & /bahdee/ & jail & /jayl/ & soft & /sawft/ \\
\hline boil & /boil/ & jam & /jam/ & soil & /soil/ \\
\hline bone & /bohn/ & jammy & /jamee/ & sold & /sohld/ \\
\hline book & /book/ & jangle & /jangul/ & soldier & /sohljur/ \\
\hline books & /books/ & jar & /jahr/ & solo & /sohloh/ \\
\hline born & /bawrn/ & jargon & /jahrgawn/ & some & /suhm/ \\
\hline borrow & /bawroh/ & jaunt & /jawnt/ & son & /suhn/ \\
\hline botch & /bawch/ & jaw & ljaw/ & song & /sawng/ \\
\hline both & /bohxh/ & jazz & /jazl & soon & /suen/ \\
\hline bottle & /bawtul/ & jazzy & /jazee/ & sorry & /sahree/ \\
\hline bounds & /bowndz/ & jeans & /jeenz/ & sort & /sawrt/ \\
\hline
\end{tabular}




\begin{tabular}{|c|c|c|c|c|c|}
\hline box & /bahks/ & jeer & /jeer/ & sound & /sownd/ \\
\hline boxer & /bahksur/ & jelly & /jelee/ & south & /sowth/ \\
\hline boxy & /bahksee/ & jemmy & /jemee/ & soy & /soi/ \\
\hline boy & /boi/ & jerk & /jerk/ & space & /spays/ \\
\hline boy & /boi/ & Jeron & /jerahn/ & speak & /speek/ \\
\hline boyish & /boiish/ & jersey & /jursee/ & special & /speshul/ \\
\hline brain & /brayn/ & jest & /jest/ & spend & /spend/ \\
\hline brains & /braynzl & jester & /jestur/ & spent & /spent/ \\
\hline branch & /branch/ & Jesus & /jeesuhs/ & spoke & /spohk/ \\
\hline branch & /branch/ & jet & /jet/ & spot & /spaht/ \\
\hline brash & /brash/ & jetty & /jetee/ & spread & /spred/ \\
\hline breach & /breech/ & Jew & /jue/ & spring & /spring/ \\
\hline bread & /bred/ & jewel & /juel/ & square & /skwer/ \\
\hline break & /brayk/ & jib & /jib/ & stalk & /stahlk/ \\
\hline bridge & /brij/ & jiffy & /jifee/ & stand & /stand/ \\
\hline briefs & /breefs/ & jig & /jig/ & star & /stahr/ \\
\hline bright & /briet & jiggle & /jigul/ & starch & /stahrch/ \\
\hline bring & /bring/ & jigsaw & /jigsaw/ & start & /stahrt/ \\
\hline broad & /brawd/ & jilt & /jilt/ & state & /stayt/ \\
\hline broke & /brohk/ & jingle & /jingul/ & station & /stayshun/ \\
\hline broken & /brohkun/ & jitter & /jittur/ & stay & /stay/ \\
\hline broth & /brahth/ & jive & /jiev/ & step & /step/ \\
\hline brought & /brawt/ & job & /jahb/ & stick & /stik/ \\
\hline brown & /brown/ & jockey & /jawkee/ & still & /stil// \\
\hline brunch & /bruhnch/ & jog & /jahg/ & stitch & /stich/ \\
\hline bucks & /buhks/ & join & /join/ & stock & /stawk/ \\
\hline buddy & /buhdee/ & joint & /joint/ & stone & /stohn/ \\
\hline bug & /buhg/ & joke & /johk/ & stood & /stood/ \\
\hline build & /bild/ & joker & /johkur/ & stop & /stahp/ \\
\hline building & /bilding/ & jolly & /jawlee/ & store & /stawr/ \\
\hline built & /bilt/ & Jon & /jahn/ & storm & /stawrm/ \\
\hline bulk & /buhlk/ & joshes & /jawshizl & story & /stawree/ \\
\hline burn & /burn/ & joy & /joi/ & straight & /strayt/ \\
\hline bus & /buhs/ & judge & /juhi/ & strange & /strayni/ \\
\hline bush & /boosh/ & jug & /juhg/ & stranger & /straynjur/ \\
\hline bushes & /booshiz/ & juice & /jues/ & stream & /streem/ \\
\hline business & /biznus/ & juke & /juek/ & street & /street/ \\
\hline busty & /buhstee/ & July & /Joolie/ & strength & /strengxh/ \\
\hline busy & /bizee/ & jumble & /juhmbul/ & strike & /striek/ \\
\hline but & /buht/ & jump & /juhmp/ & strong & /strawnq/ \\
\hline butter & /buhtur/ & June & /Juen/ & student & /stuedunt/ \\
\hline buy & /bie/ & jungle & /juhngul/ & study & /stuhdee/ \\
\hline by & /bie/ & junior & /juenyur/ & sturdy & /sturdee/ \\
\hline cake & /kayk/ & junk & /juhnk/ & subject & /suhbjikt/ \\
\hline call & /kawl/ & just & /juhst/ & succeed & /sukseed/ \\
\hline came & /kaym/ & kecks & /keks/ & success & /sukses/ \\
\hline can & /kan/ & keep & /keep/ & such & /suhch/ \\
\hline captain & /kaptun/ & kept & /kept/ & sudden & /suhdun/ \\
\hline car & /kahr/ & keshes & /keeshiz/ & suffer & /suhfur/ \\
\hline care & /ker/ & key & /kee/ & sugar & /shoogur/ \\
\hline
\end{tabular}




\begin{tabular}{|c|c|c|c|c|c|}
\hline carry & /karee/ & kicks & /kikzl & suit & /suet/ \\
\hline case & /kays/ & kidney & /kidnee/ & sulk & /suhlk/ \\
\hline cash & /kash/ & kilo & /keeloh/ & summer & /suhmur/ \\
\hline cashes & /kashiz/ & kind & /kiend/ & sun & /suhn/ \\
\hline catch & /kach/ & king & /king/ & Sunday & /Suhnday/ \\
\hline caught & /kawt/ & kiss & /kis/ & supply & /suhplie/ \\
\hline caulk & /kawlk/ & kitchen & /kichun/ & suppose & /supohz/ \\
\hline cause & /kawzl & kitty & /kitee/ & sure & /shoor/ \\
\hline cello & /cheloh/ & labor & /laybur/ & surprise & /supriez/ \\
\hline cent & /sent/ & laches & /lachizl & sweet & /sweet/ \\
\hline century & /senchuri/ & ladder & /ladur/ & switch & /swich/ \\
\hline chafe & /chayf/ & lady & /laydee/ & syntax & /sintaks/ \\
\hline chair & /cher/ & lake & /layk/ & system & /sistum/ \\
\hline chair & /cher/ & land & /land/ & table & /taybul/ \\
\hline chalk & /chawlk/ & language & /langgwij/ & tail & /tayl/ \\
\hline chance & /chans/ & large & /lahrj/ & take & /tayk/ \\
\hline chance & /chans/ & lashes & /lashiz/ & talem & /taykun/ \\
\hline change & /chang/ & last & /last/ & talk & /tawk/ \\
\hline chant & /chant/ & late & /layt/ & talks & /tawlkz/ \\
\hline chaos & /kayahs/ & laugh & /laf/ & tall & /tawl/ \\
\hline character & /karuktur/ & laughter & /laftur/ & tammy & /tamee/ \\
\hline charge & /chahrj/ & law & /law/ & taste & /tayst/ \\
\hline charm & /chahrm/ & lax & /laks/ & tasty & /taystee/ \\
\hline chase & /chays/ & lay & /lay/ & $\operatorname{tax}$ & /taks/ \\
\hline chat & /chat/ & lead & /leed/ & taxi & /taksee/ \\
\hline cheat & /cheet/ & leader & /leedur/ & teach & /teech/ \\
\hline cheer & /cheer/ & learn & /lurn/ & tear & /tir/ \\
\hline chesty & /chestee/ & least & /leest/ & tell & /tel/ \\
\hline chew & /chue/ & leave & /leev/ & ten & /ten/ \\
\hline chief & /cheef/ & leches & /lechiz/ & testy & /testee/ \\
\hline child & /chield/ & led & /led/ & thai & /tie/ \\
\hline childhood & /chieldhood/ & left & /left/ & than & /than/ \\
\hline children & /childrun/ & leg & /leg/ & thank & /xhangk/ \\
\hline chill & /chil/ & legs & /leqzI & thanks & /thanks/ \\
\hline chin & /chin/ & length & /lengxh/ & that & /that/ \\
\hline choke & /chohk/ & less & /les/ & the & /thee/ \\
\hline choose & /chuez/ & let & /let/ & theft & /theft/ \\
\hline chore & /chawr/ & letter & /letur/ & their & /ther/ \\
\hline Christ & /chriest/ & liar & /lier/ & theirs & /therz/ \\
\hline chunk & /chuhnk/ & lie & /lie/ & them & /them/ \\
\hline church & /church/ & life & /lief/ & theme & /theem/ \\
\hline churn & /churn/ & light & /liet/ & then & /then/ \\
\hline cigarette & /siguret/ & limy & /liemee/ & thence & /thens/ \\
\hline circle & /surkul/ & line & /lien/ & theory & /thirree/ \\
\hline city & /sitee/ & liquid & /likwid/ & there & /ther/ \\
\hline civics & /siviks/ & liquor & /likur/ & therefore & /therfawr/ \\
\hline class & /klas/ & list & /list/ & these & /theez/ \\
\hline clay & /klay/ & listen & /lisun/ & thesis & /theesus/ \\
\hline clean & /kleen/ & little & /litul/ & they & /thay/ \\
\hline clear & /klir/ & live & /liv/ & thick & /xhik/ \\
\hline
\end{tabular}




\begin{tabular}{|c|c|c|c|c|c|}
\hline clock & /klahk/ & loath & /lohth/ & thief & /theef/ \\
\hline close & /klohs/ & lobby & /lahbee/ & thigh & /thie/ \\
\hline cloth & /klawxh/ & Ioin & /loin/ & thin & /xhin/ \\
\hline clothes & /klohzl & loiter & /loitur/ & thing & /xhing/ \\
\hline cloud & /klowd/ & lone & /lohn/ & things & /thingz/ \\
\hline clutch & /kluhch/ & long & /lawng/ & think & /xhingk/ \\
\hline coat & /koht/ & look & /lookI & third & /thurd/ \\
\hline coil & /koil/ & Lord & /Lawrd/ & thirst & /thurst/ \\
\hline coin & /koin/ & lose & /luezl & thirteen & /xhurteen/ \\
\hline cold & /kohld/ & loss & /laws/ & thirty & /thurstee/ \\
\hline college & /kahlij/ & lost & /lawst/ & this & /this/ \\
\hline color & /kuhlur/ & lot & /laht/ & those & /thohz/ \\
\hline come & /kuhm/ & loud & /lowd/ & though & /thoh/ \\
\hline company & /kuhmpunee/ & love & /luhv/ & thought & /xhawt/ \\
\hline complete & /kumpleet/ & low & /loh/ & thousand & /xhowzund/ \\
\hline condition & /kundishun/ & lower & /lohur/ & thrall & /thral/ \\
\hline consider & /kunsidur/ & lox & /lawks/ & thrash & /thrash/ \\
\hline considerable & /kunsidurubul/ & loyal & /loiyul/ & thrawn & /thrawn/ \\
\hline contain & /kuntayn/ & lurch & /lurch/ & thread & /thred/ \\
\hline continue & /kuntinyue/ & lushes & /luhshiz/ & threat & /thret/ \\
\hline control & /kuntrohl/ & lusty & /lahbee/ & three & /xhree/ \\
\hline convey & /kohnvay/ & lynch & /linch/ & threw & /xhrue/ \\
\hline convoy & /kahnvoi/ & $\mathrm{Ma}$ & /Mah/ & thrice & /thries/ \\
\hline cook & /kook/ & machine & /musheen/ & thrill & /thril/ \\
\hline cool & /kuel/ & mad & $/ \mathrm{mad} /$ & thrive & /thriev/ \\
\hline corn & /kawrn/ & made & /mayd/ & throb & /thrahb/ \\
\hline corner & /kawnur/ & mail & /mayl/ & throe & /throh/ \\
\hline coshes & /kohshizl & mains & /maynzl & throne & /throhn/ \\
\hline cost & /kawst/ & make & /mayk/ & throng & /thrawng/ \\
\hline could & /kood/ & man & /man/ & through & Ixhrue/ \\
\hline count & /kownt/ & manner & /manur/ & throve & /throhv/ \\
\hline country & /kuhntree/ & many & /meni/ & throw & /xhroh/ \\
\hline course & /kawrs/ & March & /mahrch/ & thrown & /xhrohn/ \\
\hline cover & /kuhvur/ & mark & /mahrk/ & thru & /thrue/ \\
\hline $\operatorname{cox}$ & /kahks/ & market & /mahrkut/ & thrust & /thruhst/ \\
\hline crash & /krash/ & marque & /mahrk/ & thug & /thuhg/ \\
\hline crips & /krips/ & marry & /maree/ & thumb & /thuhm/ \\
\hline cross & /kraws/ & marsh & /mawrsh/ & thumbs & /thuhmz/ \\
\hline crowd & /krowd/ & master & /mastur/ & thus & /thuhs/ \\
\hline crusty & /crestee/ & material & /mutiriul/ & tiches & /tichiz/ \\
\hline cry & /krie/ & maths & /mathz/ & tidy & /tiedee/ \\
\hline cup & /kuhp/ & matter & /matur/ & tie & /tie/ \\
\hline cut & /kuht/ & maxim & /maksem/ & till & $/$ til/ \\
\hline daddy & /dadee/ & May & /May/ & time & /tiem/ \\
\hline daily & /daylee/ & mayor & /mayur/ & to & /tue/ \\
\hline dance & /dans/ & me & /mee/ & today & /tooday/ \\
\hline dare & /der/ & mean & /meen/ & together & /toogethur/ \\
\hline dark & /dahrk/ & means & /meenz/ & toil & /toil/ \\
\hline darts & /dahrtz/ & measure & /mezhur/ & told & /tohld/ \\
\hline dashes & /dashiz/ & meat & /meet/ & tommy & /tahmee/ \\
\hline
\end{tabular}




\begin{tabular}{|c|c|c|c|c|c|}
\hline date & /dayt/ & meaty & /meetee/ & tomorrow & /toomahroh/ \\
\hline daughter & /dawtur/ & meet & /meet/ & too & /tue/ \\
\hline day & /day/ & meeting & /meeting/ & took & /took/ \\
\hline dayglo & /daygloh/ & member & /membur/ & tooth & /tueth/ \\
\hline dead & /ded/ & men & /men/ & torch & /tawrch/ \\
\hline deal & /deel/ & met & /met/ & tore & /tawr/ \\
\hline dear & /dir/ & method & /mexhud/ & touch & /tuhch/ \\
\hline December & /Disembur/ & meths & /methz/ & toward & /tawrd/ \\
\hline decide & /disied/ & middle & /midul/ & town & /town/ \\
\hline decks & /dekzI & might & /miet/ & toxic & /tawksik/ \\
\hline decoy & /deekoi/ & mile & /miel/ & toy & /toi/ \\
\hline deep & /deep/ & milk & /milk/ & tracks & /traks/ \\
\hline degree & /digree/ & million & /milyun/ & trade & /trayd/ \\
\hline deity & /deetee/ & mind & /miend/ & train & /trayn/ \\
\hline delight & /diliet/ & mine & /mien/ & training & /trayning/ \\
\hline demand & /dimand/ & minute & /minit/ & travel & /travul/ \\
\hline deploy & /deeploi/ & miss & /mis/ & tree & /tree/ \\
\hline depth & /depth/ & mister & /mistur/ & tried & /tried/ \\
\hline desire & /dizier/ & misty & /mistee/ & tries & /triez/ \\
\hline destroy & distroi/ & $\operatorname{mix}$ & /miks/ & trip & /trip/ \\
\hline detach & /deetach/ & modern & /mawdurn/ & trouble & /truhbul/ \\
\hline detox & /deetahks/ & moist & /moist/ & trust & /truhst/ \\
\hline device & /divies/ & Monday & /Muhnday/ & truth & /trueth/ \\
\hline devoid & /deevoid/ & money & /mohnee/ & try & /trie/ \\
\hline dibs & /dibz/ & month & /muhnxh/ & Tuesday & /Tuezday/ \\
\hline dicey & /diesee/ & moody & /muedee/ & turfs & /turfs/ \\
\hline did & /did/ & moon & /muen/ & turn & /turn/ \\
\hline die & /die. & more & /mawr/ & twelve & /twelv/ \\
\hline difference & /difruns/ & morning & /mawrning/ & twenty & /twentee/ \\
\hline different & /difrunt/ & mosque & /mahsk/ & twins & /twinz/ \\
\hline difficult & /difukult/ & most & /mohst/ & twitch & /twich/ \\
\hline $\operatorname{dig}$ & /dig/ & moth & /mawth/ & two & /tue/ \\
\hline digs & /digz/ & mother & /muhthur/ & udder & /uhdur/ \\
\hline dinner & /dinur/ & mountain & /mowntin/ & uglier & /uhgleeur/ \\
\hline dioxin & /deeahksin/ & mouth & /mowxh/ & uglis & /uhglis/ \\
\hline direct & /direkt/ & move & /muev/ & ugly & /uhglee/ \\
\hline dirty & /dirtee/ & movement & /muevmunt/ & ulcer & lawlsurl \\
\hline discover & /diskuhvur/ & $\operatorname{moxa}$ & /mohksu/ & ulema & /ulemu/ \\
\hline dish & /dish/ & much & /muhch/ & ulna & lawinu/ \\
\hline dishes & /dishiz/ & mud & /muhd/ & ultima & /awlteemu/ \\
\hline dismay & /dismay/ & munch & /muhnch/ & ultra & /awltru/ \\
\hline distance & /distuns/ & music & /myuezik/ & umbel & /uhmbul/ \\
\hline distant & /distunt/ & must & /muhst/ & umber & /uhmbur/ \\
\hline ditch & /dich/ & my & /mie/ & umpire & /uhmpieur/ \\
\hline divide & /divied/ & nail & /nayl/ & uncle & /uhngkul/ \\
\hline & /due/ & nasty & /nastee/ & under & /uhndur/ \\
\hline doctor & /dahktur/ & nation & /nayshun/ & understand & /uhndurstand/ \\
\hline does & /duhz/ & nature & /naychur/ & understood & /uhndurstood/ \\
\hline dog & /dawg/ & near & Inir/ & until & /until/ \\
\hline doing & /dueing/ & nearly & /nirli/ & up & /uhp/ \\
\hline
\end{tabular}




\begin{tabular}{|c|c|c|c|c|c|}
\hline dollar & /dahlur/ & necessary & /nesuseri/ & upon & /upahn/ \\
\hline done & /duhn/ & neck & Inek & urban & /urban/ \\
\hline donkey & /duhnkee/ & need & /need/ & urchin & /urchin/ \\
\hline don't & /dohnt/ & needle & /needul/ & urea & /ureeu/ \\
\hline door & /dawr/ & needs & /needz/ & urge & /urg/ \\
\hline dormie & /dawrmee/ & needy & /needee/ & urgent & /urgent/ \\
\hline dormy & /dawrmee/ & neighbor & /naybur/ & urine & /yurin/ \\
\hline double & /duhbul/ & neither & /neethur/ & us & /uns/ \\
\hline doubt & /dowt/ & nerve & Inurv/ & use & /yuez/ \\
\hline down & /down/ & never & /nevur/ & use & /yues/ \\
\hline dream & /dreem/ & new & /nue/ & usual & /yuezhooul/ \\
\hline dregs & /dregz/ & news & /nuez/ & vac & /vac/ \\
\hline dress & /dres/ & next & /nekst/ & vacuum & /vakuem/ \\
\hline dried & /dried/ & nice & /nies/ & vagina & /vahjienu/ \\
\hline drink & /dringk/ & niece & /nees/ & vague & /vayg/ \\
\hline drive & /driev/ & night & /niet/ & vain & /vayn/ \\
\hline drop & /drahp/ & nine & /nien/ & valet & /valay/ \\
\hline dry & /drie/ & ninth & /nienth/ & valid & /valid/ \\
\hline dubs & /duhbz/ & no & /noh/ & valley & /valee/ \\
\hline duck & /duhk/ & noise & /noizl & value & /valyue/ \\
\hline dummy & /duhmee/ & none & /nuhn/ & valve & /valv/ \\
\hline during & /dooring/ & noon & /nuen/ & vamp & /vamp/ \\
\hline dusk & /duhsk/ & nor & /nawr/ & van & /van/ \\
\hline dusty & /duhstee/ & north & /nawrxh/ & vandal & /vandul/ \\
\hline duty & /duetee/ & nose & /nohz/ & vanish & /vanish/ \\
\hline dwarfs & /dwawrfs/ & not & /naht/ & vapid & /vaypid/ \\
\hline each & /eech/ & notch & /nawch/ & vapor & /vaypur/ \\
\hline ear & /ir/ & note & /noht/ & various & /varius/ \\
\hline early & /urlee/ & nothing & /nuhxhing/ & vary & /veree/ \\
\hline earth & /urxh/ & notice & /nohtis/ & vast & /vast/ \\
\hline east & /eest/ & November & /NOHvembur/ & vault & /vawlt/ \\
\hline easy & /eezee/ & now & /now/ & vector & /vektur/ \\
\hline eat & /eet/ & number & /nuhmbur/ & veer & /veer/ \\
\hline edge & lej/ & nutty & /nuhtee/ & vegan & /veegin/ \\
\hline effort & lefurt/ & nylons & /nielahnz/ & vein & /vayn/ \\
\hline egg & leg/ & oath & lohth/ & venom & /venuhm/ \\
\hline eight & layt/ & oats & lohts/ & vent & /vent/ \\
\hline either & /eethur/ & object & /ahbjekt/ & verb & /vurb/ \\
\hline electric & /ulektrik/ & ocean & /ohshun/ & verbs & /vurbz/ \\
\hline electricity & /ulektrisitee/ & oches & /ohchiz/ & verse & /verzl \\
\hline elk & lelk/ & October & /Ahktohbur/ & very & /veree/ \\
\hline else & lels/ & odds & lahdz/ & vet & /vet/ \\
\hline Emmy & lemee/ & of & /uhv/ & via & /veeuh/ \\
\hline employ & /emploi/ & off & lawf/ & vice & /vies/ \\
\hline end & /end/ & offer & /awfur/ & video & /videeoh/ \\
\hline ends & lendz/ & office & /awfis/ & view & Ivyue/ \\
\hline enemy & lenumee/ & often & lawfun/ & view & Ivue/ \\
\hline English & /ingglish/ & oh & loh/ & vile & /viel/ \\
\hline enjoy & /injoi/ & oil & /oil/ & visa & /veezu/ \\
\hline enough & linuhf & oil & /oil/ & visit & /vizit/ \\
\hline
\end{tabular}




\begin{tabular}{|c|c|c|c|c|c|}
\hline enter & /entur/ & oily & /oilee/ & vocal & /vohkul/ \\
\hline epoxy & leepawksee/ & old & /ohld/ & vogue & /vohg/ \\
\hline equal & leekwul/ & on & lahn/ & voice & /vois/ \\
\hline equip & /eekwip/ & only & /ohnlee/ & void & /void/ \\
\hline escape & /uskayp/ & oogamy & /uegamee/ & vomit & /vawmit/ \\
\hline etches & /etchizl & open & /ohpun/ & vote & /voht/ \\
\hline ethics & /ethiks/ & opinion & /upinyun/ & vouch & /vowch/ \\
\hline even & /eevun/ & or & lawr/ & voyage & /voiyeg/ \\
\hline evening & leevning/ & order & lawrdur/ & voyeur & /voiyur/ \\
\hline ever & levur/ & orderly & /awrdurli/ & wagon & /wagun/ \\
\hline every & /evree/ & other & /uhthur/ & wait & /wayt/ \\
\hline exact & /iksakt/ & ought & /awt/ & walk & /wawk/ \\
\hline exalt & /ikawlt/ & our & lowr/ & wall & /wawl/ \\
\hline exam & /iksam/ & out & lowt/ & want & /wawnt/ \\
\hline excel & /ikssel/ & outer & lowtur/ & war & /wawr/ \\
\hline except & leksept/ & outside & /owtsied/ & warm & /wawrm/ \\
\hline exert & /iksurt/ & over & /ohvur/ & was & /wuhz/ \\
\hline exile & /eksiel/ & ovolo & /ohvohlu/ & wash & /wahsh/ \\
\hline exit & /eksit/ & own & lohn/ & wash & /wahsh/ \\
\hline expect & /ikspekt/ & ox & lawks/ & washes & /washiz/ \\
\hline experience & /ikspiriuns/ & oxen & lawksen/ & watch & /wahch/ \\
\hline explain & /eksplayn/ & oxide & /awksied/ & water & /wahtur/ \\
\hline eye & liel & page & /payj/ & wave & /wayv/ \\
\hline face & /fays/ & paid & /payd/ & wax & /waks/ \\
\hline fact & /fakt/ & pain & /payn/ & way & /wayl \\
\hline fail & /fayl/ & part & /pahrt/ & we & /wee/ \\
\hline fair & /fer/ & partial & /pahrshul/ & weak & /week/ \\
\hline faith & /fayth/ & party & /pahrtee/ & wear & /wer/ \\
\hline fall & /fawl/ & pass & /pas/ & weather & /wethur/ \\
\hline family & /famulee/ & past & /past/ & wedge & /wej/ \\
\hline famous & /faymus/ & pasty & /paystee/ & Wednesday & /Wenzday/ \\
\hline fancy & /fansee/ & patch & /pach/ & weds & /wedz/ \\
\hline far & /fahr/ & pay & /pay/ & week & /week/ \\
\hline farm & /fahrm/ & peace & /pees/ & weight & /wayt/ \\
\hline fast & /fast/ & peach & /peech/ & welcome & /welkum/ \\
\hline fat & /fat/ & pegs & /pegz/ & well & /wel/ \\
\hline father & /fahthur/ & people & /peepul/ & went & /went/ \\
\hline fatty & /fatee/ & perfect & /purfikt/ & west & /west/ \\
\hline favor & /fayvur/ & perhaps & /purhaps/ & wet & /wet/ \\
\hline fax & /faks/ & period & /piriud/ & what & /whuht/ \\
\hline fear & /fir/ & person & /pursun/ & wheat & /wheet/ \\
\hline February & /Febrooeree/ & phag & /fag/ & wheel & /wheel/ \\
\hline feed & /feed/ & phage & /fayg/ & whelk & /welk/ \\
\hline feel & /feel/ & pharm & /fahrm/ & when & /when/ \\
\hline feet & /feet/ & phase & /fays/ & where & /wher/ \\
\hline feisty & /fiestee/ & phatic & /fatik/ & whether & /whethur/ \\
\hline fell & /fel/ & phenol & /feenul/ & which & /which/ \\
\hline fellow & /feloh/ & phenyl & /feeniel/ & while & /whiel/ \\
\hline felt & /felt/ & phew & /pue/ & white & /whiet/ \\
\hline fence & /fens/ & phi & /fie/ & whole & /hohl/ \\
\hline
\end{tabular}




\begin{tabular}{|c|c|c|c|c|c|}
\hline fetch & /fech/ & phial & /fieul/ & whom & /huem/ \\
\hline few & /fyue/ & phiz & /fiz/ & whose & /huez/ \\
\hline field & /feeld/ & phlox & /flahks/ & why & /whie/ \\
\hline fifteen & /fifteen/ & phobia & /fohbeeu/ & width & /width/ \\
\hline fifth & /fifxh/ & phoebe & /feebee/ & wife & /wief/ \\
\hline fifty & /fiftee/ & phon & /fahn/ & wild & /wield/ \\
\hline fight & /fiet/ & phone & /fohn/ & will & /wil/ \\
\hline figure & /figyur/ & phonic & /fahnik/ & win & /win/ \\
\hline fill & /fil/ & phony & /fohnee/ & wind & /wind/ \\
\hline filo & /filoh/ & phot & /fawt/ & window & /windoh/ \\
\hline filth & /filth/ & photo & /fohtoh/ & wing & /wing/ \\
\hline find & /fiend/ & photon & /fohtahn/ & wings & /wingz/ \\
\hline fine & /fien/ & phrase & /frays/ & winter & /wintur/ \\
\hline finger & /finggur/ & phut & /fuht/ & wise & /wiez/ \\
\hline finish & /finish/ & phyla & /fielu/ & wish & /wish/ \\
\hline finish & /finish/ & physic & /fisik/ & wishes & /wishiz/ \\
\hline fire & /fier/ & pick & /pik/ & with & /with/ \\
\hline firm & /furm/ & picture & /pikchur/ & within & /within/ \\
\hline first & /furst/ & piece & /pees/ & without & /withowt/ \\
\hline fish & /fish/ & pinch & /pinch/ & witty & /witee/ \\
\hline fishes & /fishiz/ & pique & /pik/ & woman & /woomun/ \\
\hline fit & /fit/ & pith & /pith/ & women & /wimin/ \\
\hline five & /fiev/ & pity & /pitee/ & won & /wuhn/ \\
\hline fix & /fiks/ & pixel & /piksul/ & wonder & /wuhndur/ \\
\hline fix & /fiks/ & place & /plays/ & wood & /wood/ \\
\hline fixed & /fikst/ & plain & /playn/ & word & /wurd/ \\
\hline flabby & /flabee/ & plan & /plan/ & wore & /wawr/ \\
\hline flier & /flier/ & plant & /plant/ & work & /wurk/ \\
\hline floor & /flawr/ & plaque & /plak/ & world & /wurld/ \\
\hline flower & /flowur/ & play & /play/ & worn & /wawrn/ \\
\hline fly & /flie/ & pleasant & /plezunt/ & worth & /wurxh/ \\
\hline foamy & /fohmee/ & please & /pleez/ & would & /wood/ \\
\hline foible & /foibul/ & pleasure & /plezhur/ & write & /riet/ \\
\hline foil & /foil/ & ploy & /ploi/ & written & /writun/ \\
\hline folk & /fohlk/ & poach & /pohch/ & wrong & /rawng/ \\
\hline follow & /fawloh/ & point & /point/ & wrote & /roht/ \\
\hline food & /fued/ & poise & /pois/ & yacht & /yaht/ \\
\hline fool & /fuel/ & polo & /pohloh/ & yack & lyak/ \\
\hline foot & /foot/ & poor & /poor/ & yah & /yah/ \\
\hline for & /fawr/ & position & /puzishun/ & yahoo & /yahhue/ \\
\hline force & /fawrs/ & possible & /pahsubul/ & yajur & /yahjur/ \\
\hline foreign & /fawrun/ & pot & /paht/ & yak & lyak/ \\
\hline forest & /fawrust/ & pouch & /powch/ & yakut & /yakuet/ \\
\hline forever & /fawrevur/ & power & /powur/ & yale & /yayl/ \\
\hline forget & /furget/ & pox & /pawks/ & yam & /yam/ \\
\hline form & /fawrm/ & prepare & /priper/ & yang & /yang/ \\
\hline fortieth & /fawrtiuxh/ & present & /presunt/ & yank & /yank/ \\
\hline forty & /fawrtee/ & president & /prezudunt/ & Yankee & /yankee/ \\
\hline forward & /fawrwurd/ & press & /pres/ & yap & /yap/ \\
\hline found & /fownd/ & pretty & /pritee/ & yарру & /yappee/ \\
\hline
\end{tabular}




\begin{tabular}{|c|c|c|c|c|c|}
\hline four & /fawr/ & prey & /pray/ & yard & /yahrd/ \\
\hline fousty & /fowstee/ & price & /pries/ & yarn & /yawrn/ \\
\hline fox & /fahks/ & probably & /prahbubli/ & yate & /yayt/ \\
\hline foxed & /fahkst/ & problem & /prahblum/ & yawl & /yawl/ \\
\hline foxy & /fahksee/ & produce & /prohdues/ & yawn & /yawn/ \\
\hline foyer & /foiyur/ & promise & /prahmis/ & ye & /yee/ \\
\hline fray & /fray/ & proud & /prowd/ & year & /yir/ \\
\hline free & /free/ & prove & /pruev/ & yearly & /yeerlee/ \\
\hline fresh & /fresh/ & public & /puhblik & yearn & /yurn/ \\
\hline fresh & /fresh/ & pull & /pool/ & years & /yirzl \\
\hline Friday & /Frieday/ & pure & /pyoor/ & yeast & /yeest/ \\
\hline friend & /frend/ & push & /poosh/ & yell & /yel/ \\
\hline from & /fruhm/ & pushes & /pooshiz/ & yellow & /yeloh/ \\
\hline front & /fruhnt/ & put & /poot/ & yellow & /yelloh/ \\
\hline froth & /frawth/ & pygmy & /pigmee/ & yelp & /yelp/ \\
\hline full & /fool/ & pyramid & /peeramid/ & yen & /yen/ \\
\hline further & /furthur/ & qua & /kwa/ & yes & /yes/ \\
\hline fusty & /fuhstee/ & quad & /kwad/ & yesterday & /yesturday/ \\
\hline gain & /gayn/ & quaff & /kwaf/ & yet & /yet/ \\
\hline game & /gaym/ & quag & /kwag/ & yew & /yuel \\
\hline garden & /gahrdun/ & quail & /kwayl/ & yiddish & /yiddish/ \\
\hline gashes & /gashiz/ & quake & /kwayk/ & yield & /yild/ \\
\hline gate & /gayt/ & qualm & /kwawlm/ & yo & /yoh/ \\
\hline gather & /gathur/ & quart & /kwawrt/ & yob & /yahb/ \\
\hline gave & /gayv/ & quarter & /kwawrtur/ & yodal & /yohdul/ \\
\hline gay & /gayl & quartz & /kwartz/ & yoga & /yohgu/ \\
\hline general & /jenurul/ & quash & /kwahsh/ & Yogi & /yohgee/ \\
\hline gentle & /jentul/ & quasi & /kwahzee/ & yoke & /yohk/ \\
\hline gentleman & /jentulmun/ & quay & /kway/ & yolk & /yohlk/ \\
\hline gents & /jents/ & queen & /kween/ & yonder & /yahndur/ \\
\hline get & /get/ & queen & /kween/ & yore & /yawr/ \\
\hline gift & /gift/ & queer & /kweer/ & you & lyue/ \\
\hline girl & /gurl/ & quell & /kwel/ & young & /yuhng/ \\
\hline give & /giv/ & query & /kweeree/ & your & /yawr/ \\
\hline glad & /glad/ & quest & /kwest/ & yours & /yawrzl \\
\hline glass & /glas/ & question & /kweschun/ & youth & /yueth/ \\
\hline gloom & /gluem/ & queue & /kwue/ & yummy & /yuhmee/ \\
\hline gloomy & /gluemee/ & quick & /kwik/ & zag & /zag/ \\
\hline glossary & /glawsuree/ & quiet & /kwieut/ & Zaire & /zieir/ \\
\hline gnash & /nash/ & quiff & /kwif/ & zakat & /zahkat/ \\
\hline go & /goh/ & quilt & /kwilt/ & zany & /zaynee/ \\
\hline God & /Gahd/ & quip & /kwip/ & zap & /zap/ \\
\hline goes & /gohz/ & quirk & /kwirk/ & zeal & /zeel/ \\
\hline gold & /gohld/ & quite & /kwiet/ & zealot & /zeelawt/ \\
\hline gone & /gawn/ & quiz & /kwiz/ & zebra & /zeebruh/ \\
\hline good & /good/ & quota & /kwohtu/ & zebu & /zebue/ \\
\hline goodbye & /goodbie/ & quote & /kwoht/ & zed & /zed/ \\
\hline got & /gaht/ & race & /rays/ & zee & /zee/ \\
\hline govern & /guhvurn/ & rags & /ragz/ & Zen & /zen/ \\
\hline grain & /grayn/ & rain & /rayn/ & zest & /zest/ \\
\hline
\end{tabular}




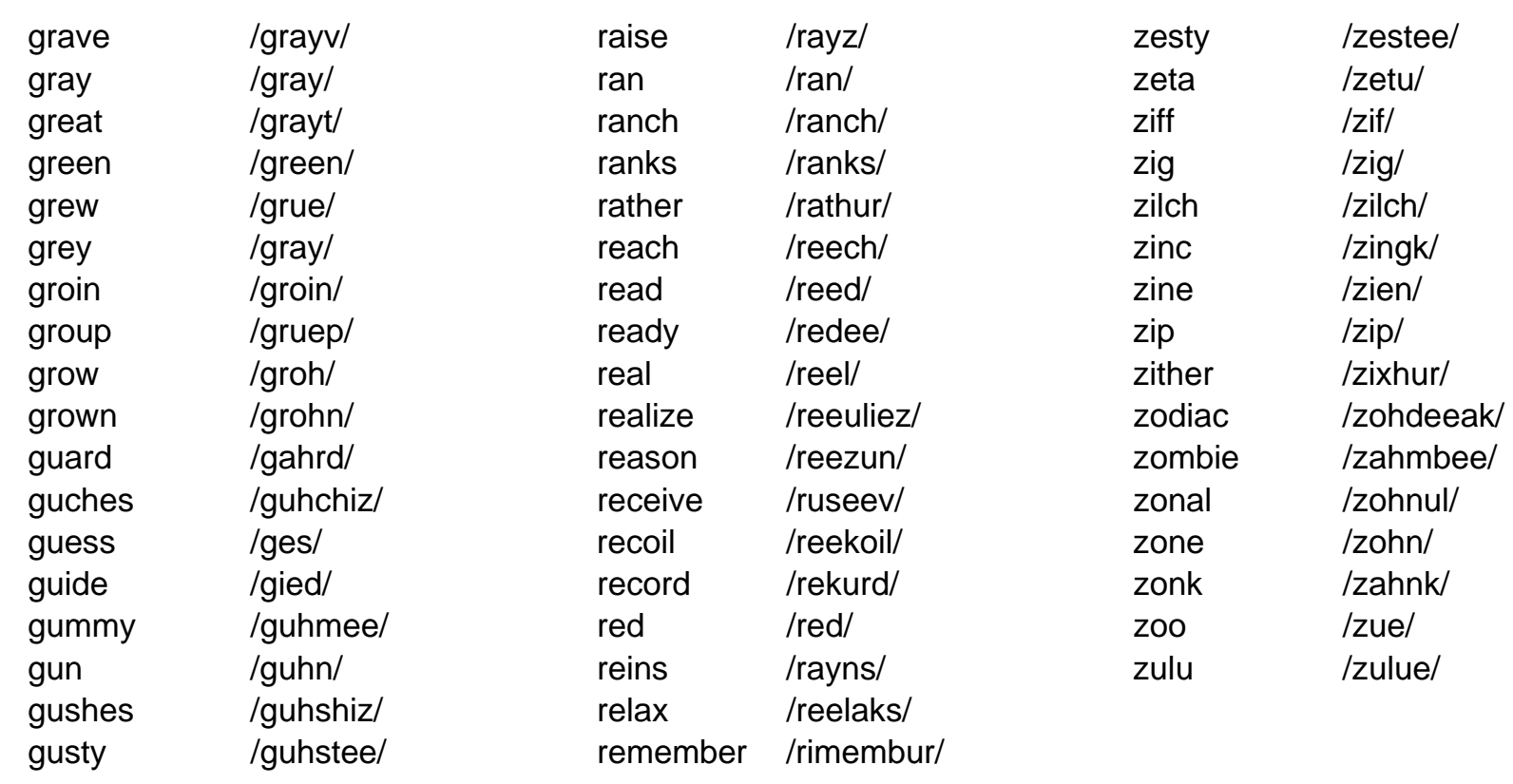




\section{Appendix D - MBROLA Program Description}

The MBROLA program is designed to take four variables as inputs. The inputs to the MBROLA program are the phoneme, the phoneme duration, the pitch pattern point, and the fundamental frequency. The program only needs the phoneme and the duration to produce speech. If the user wants to add fundamental frequency values, the program must have a corresponding pitch pattern point. The program can handle up to 20 pitch pattern point and fundamental frequency pairs.

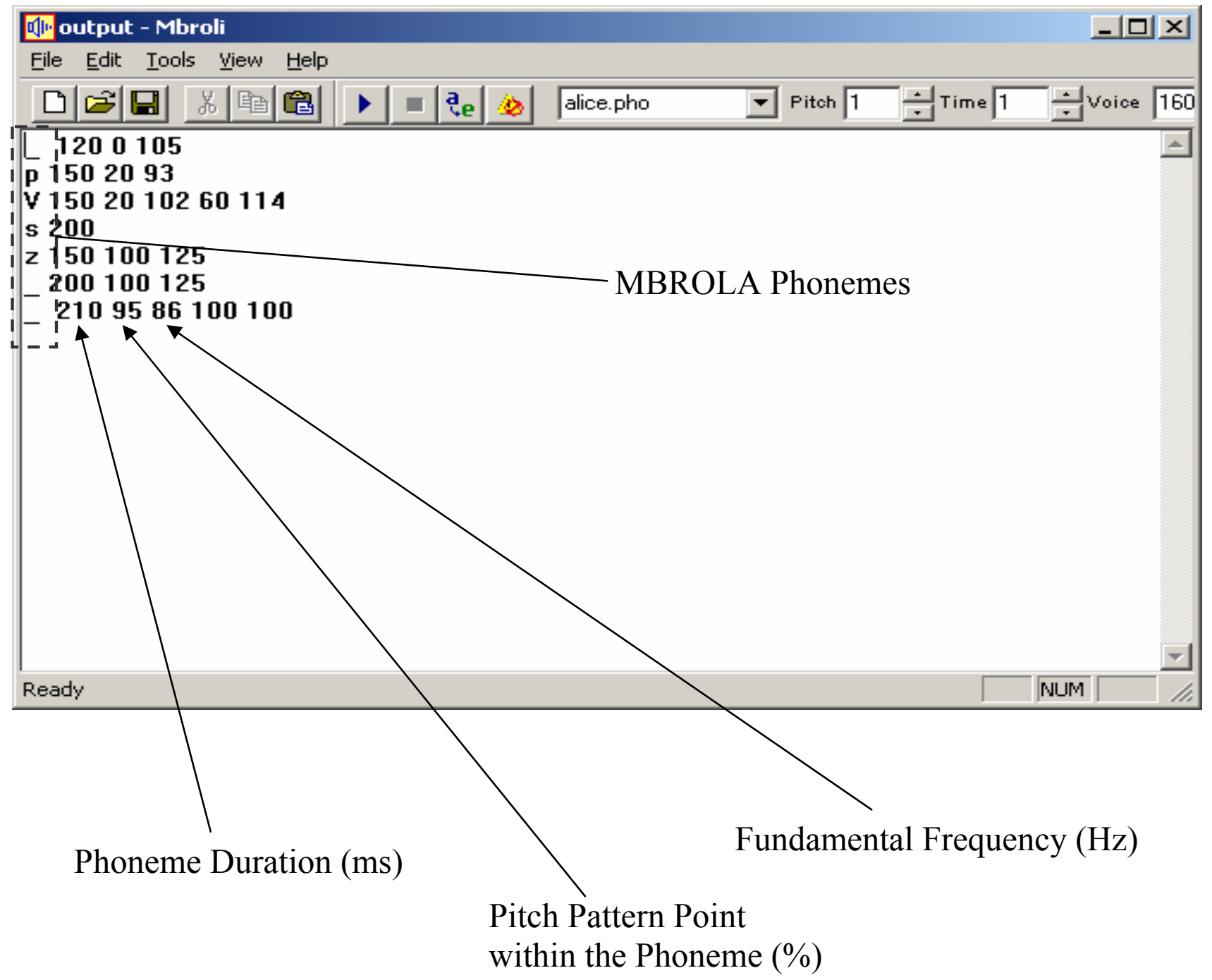




\section{Appendix E - Source Code}

\section{JonTalk Flow Chart}

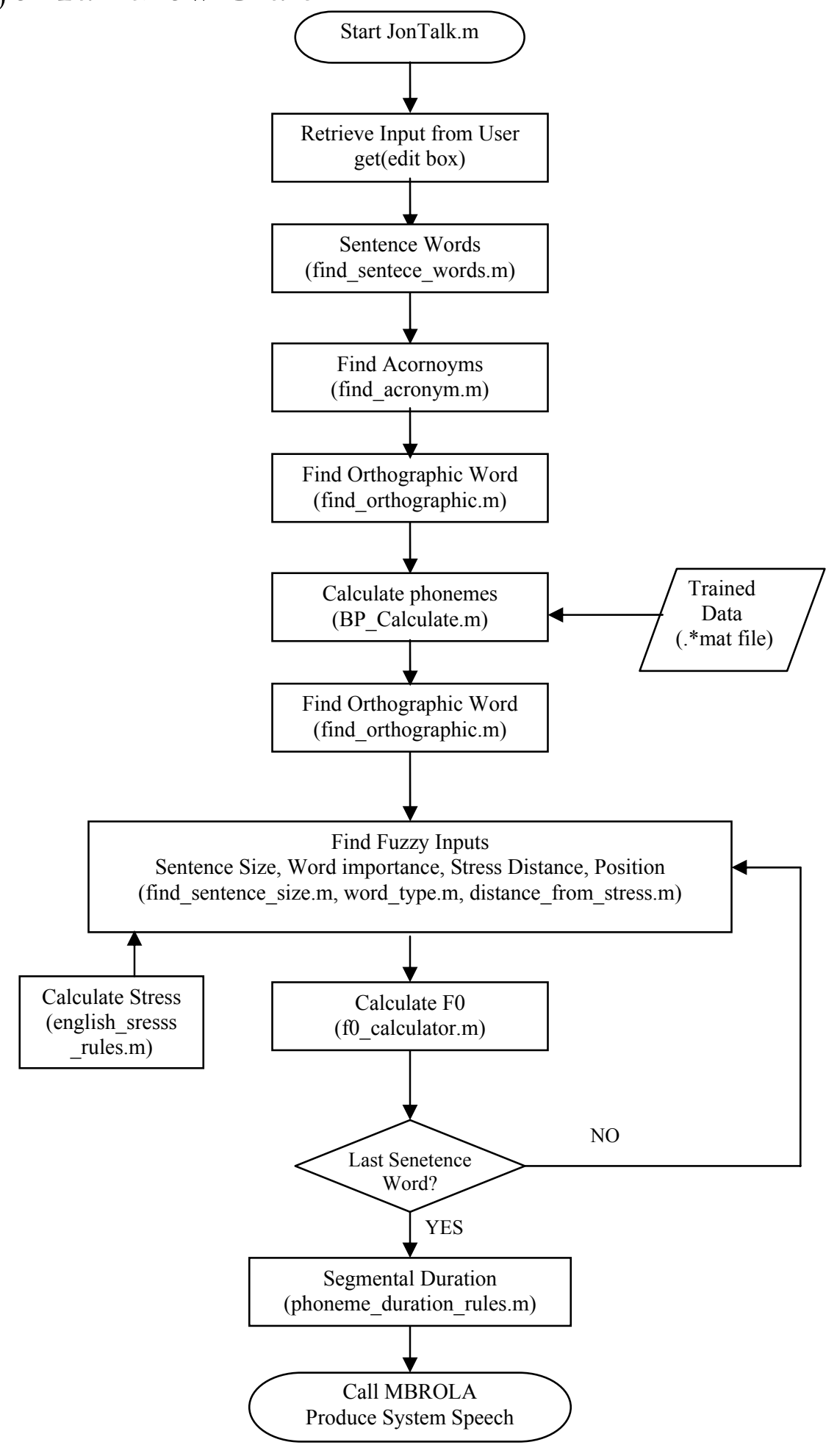




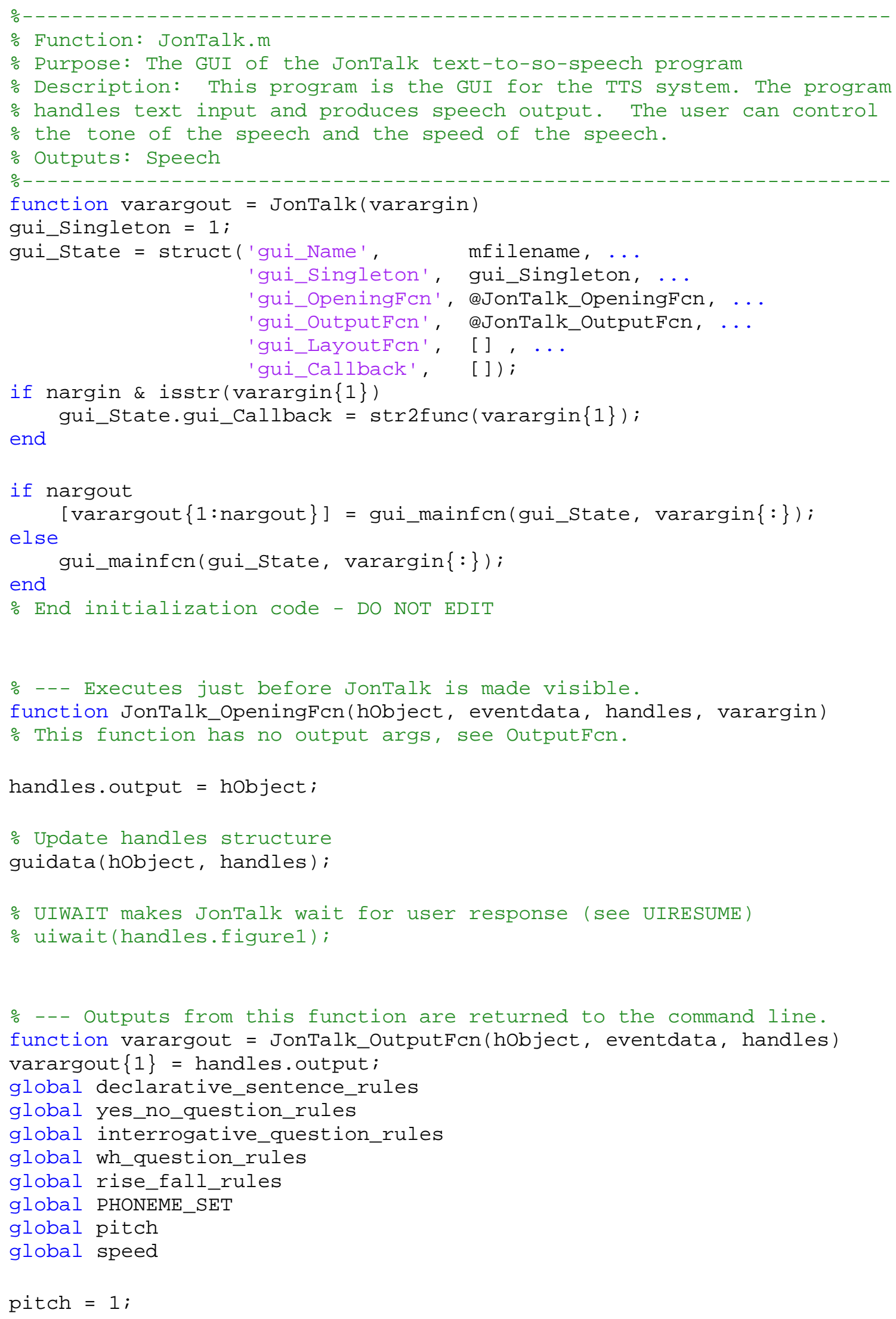




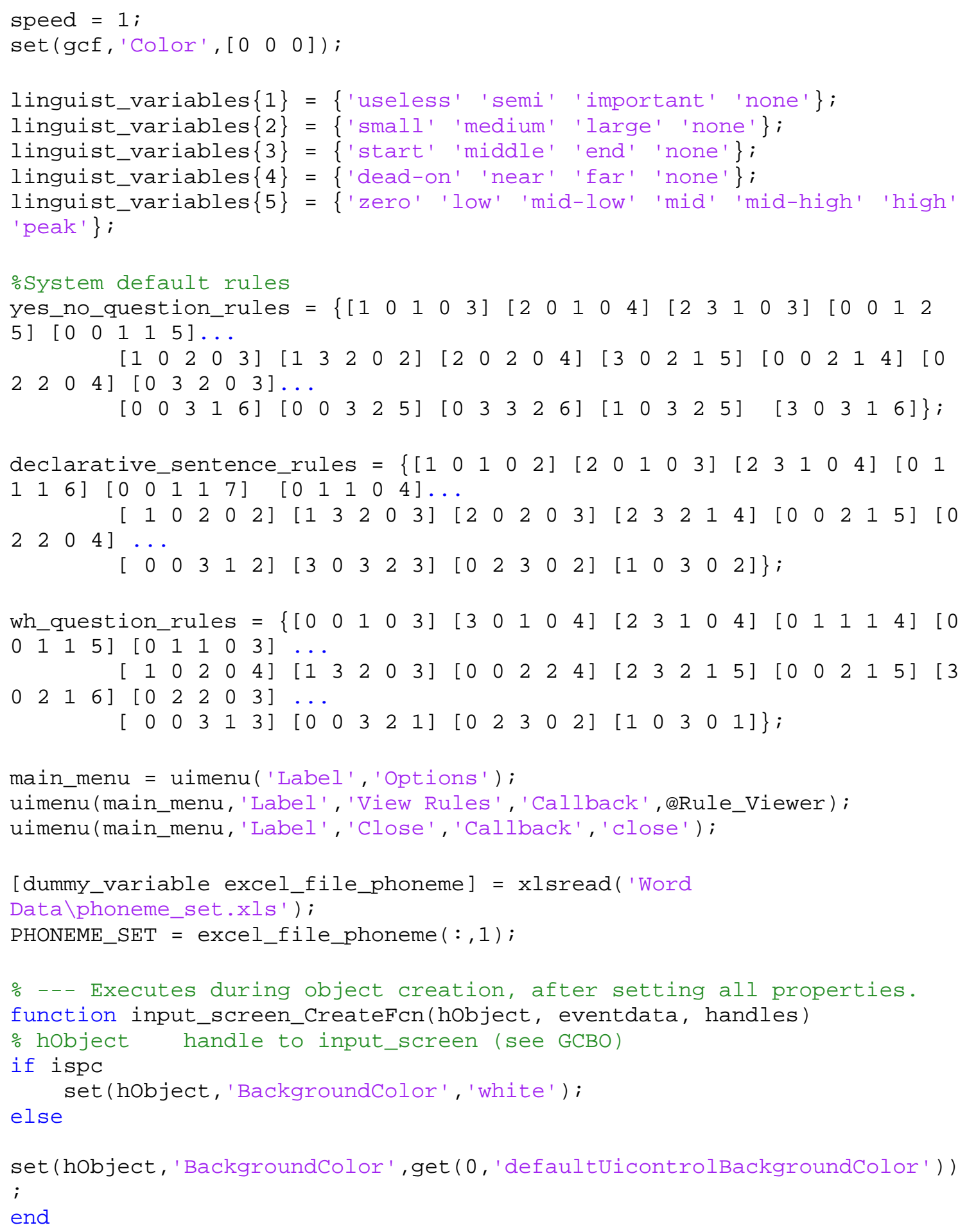




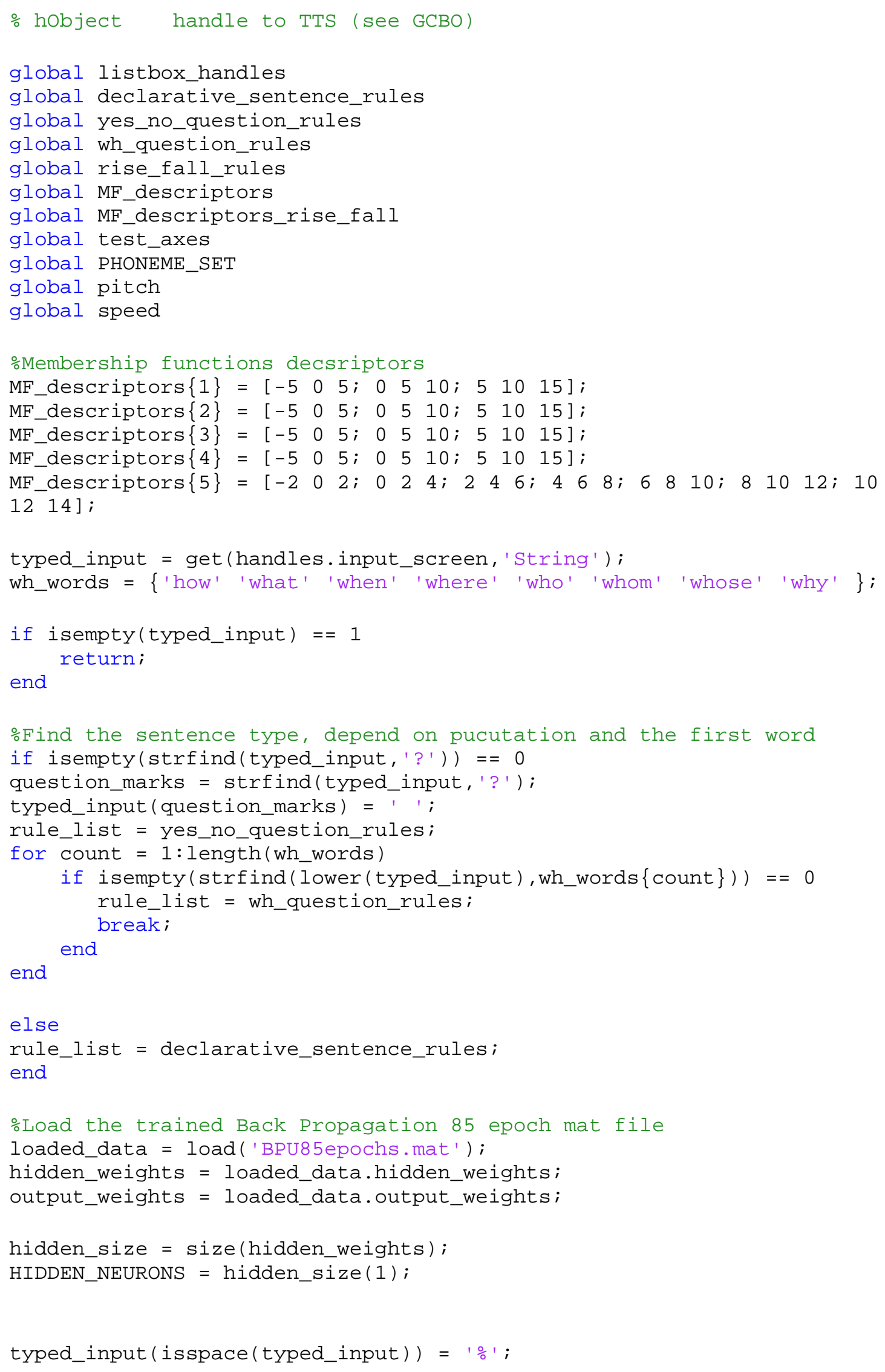




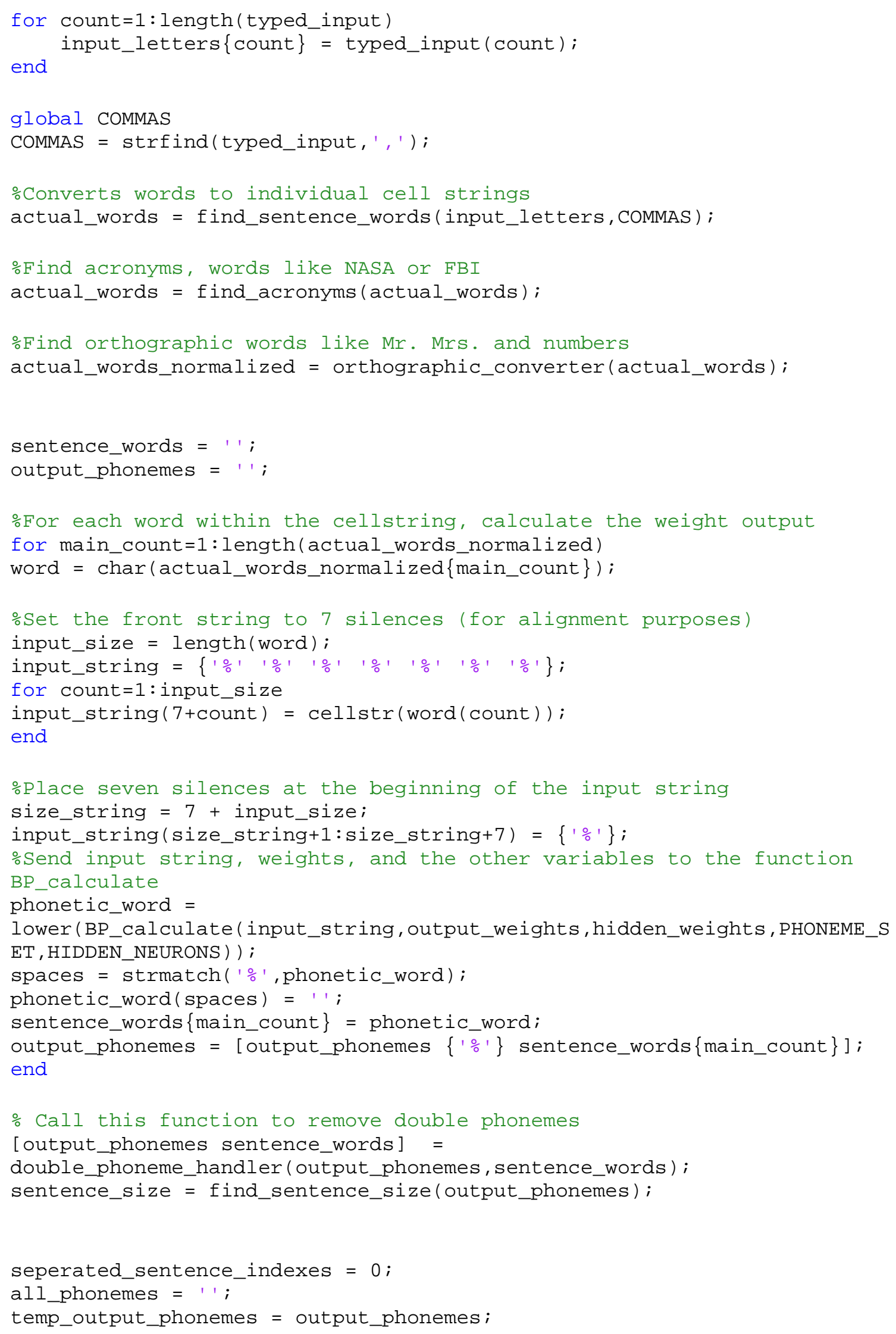




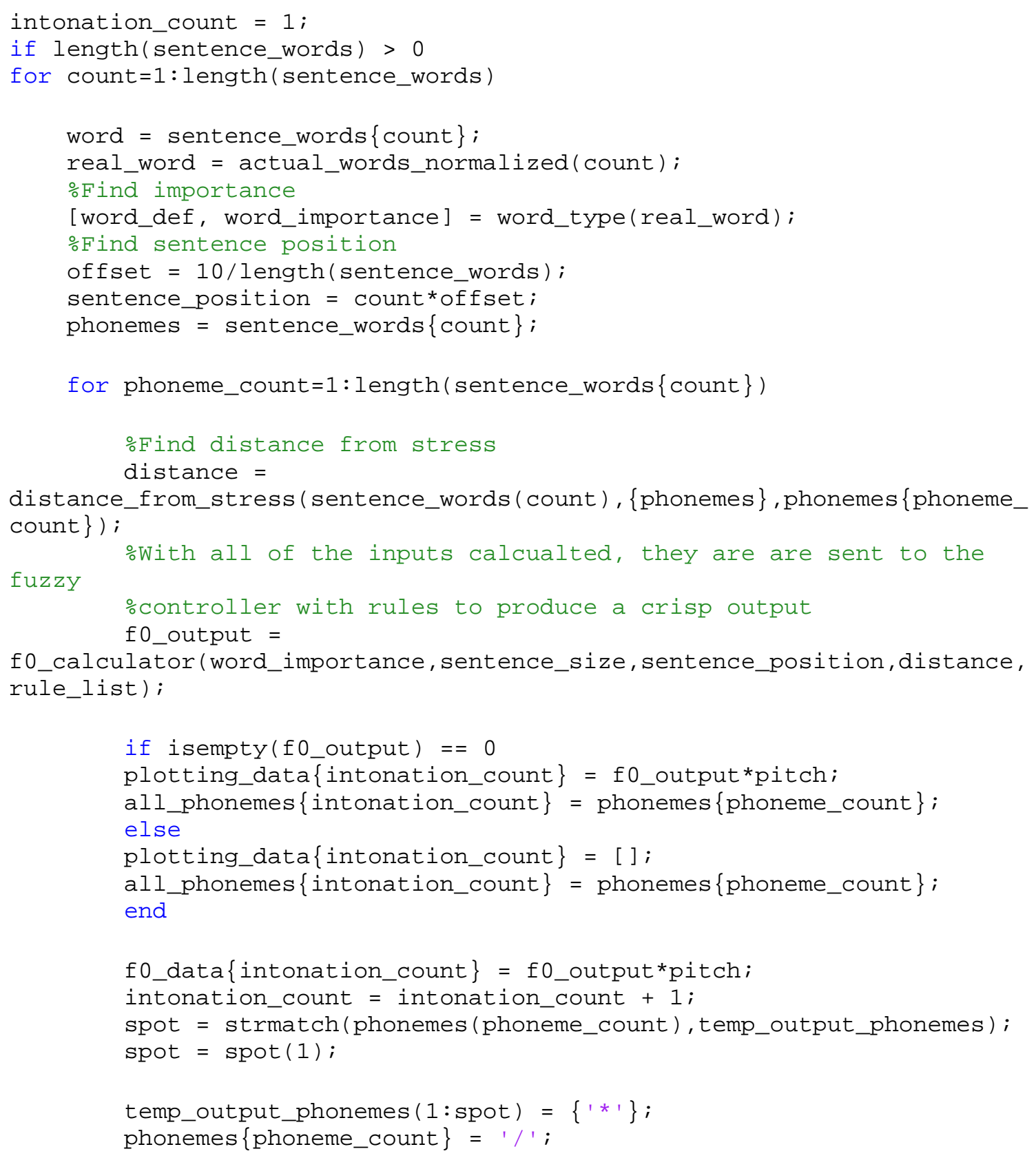




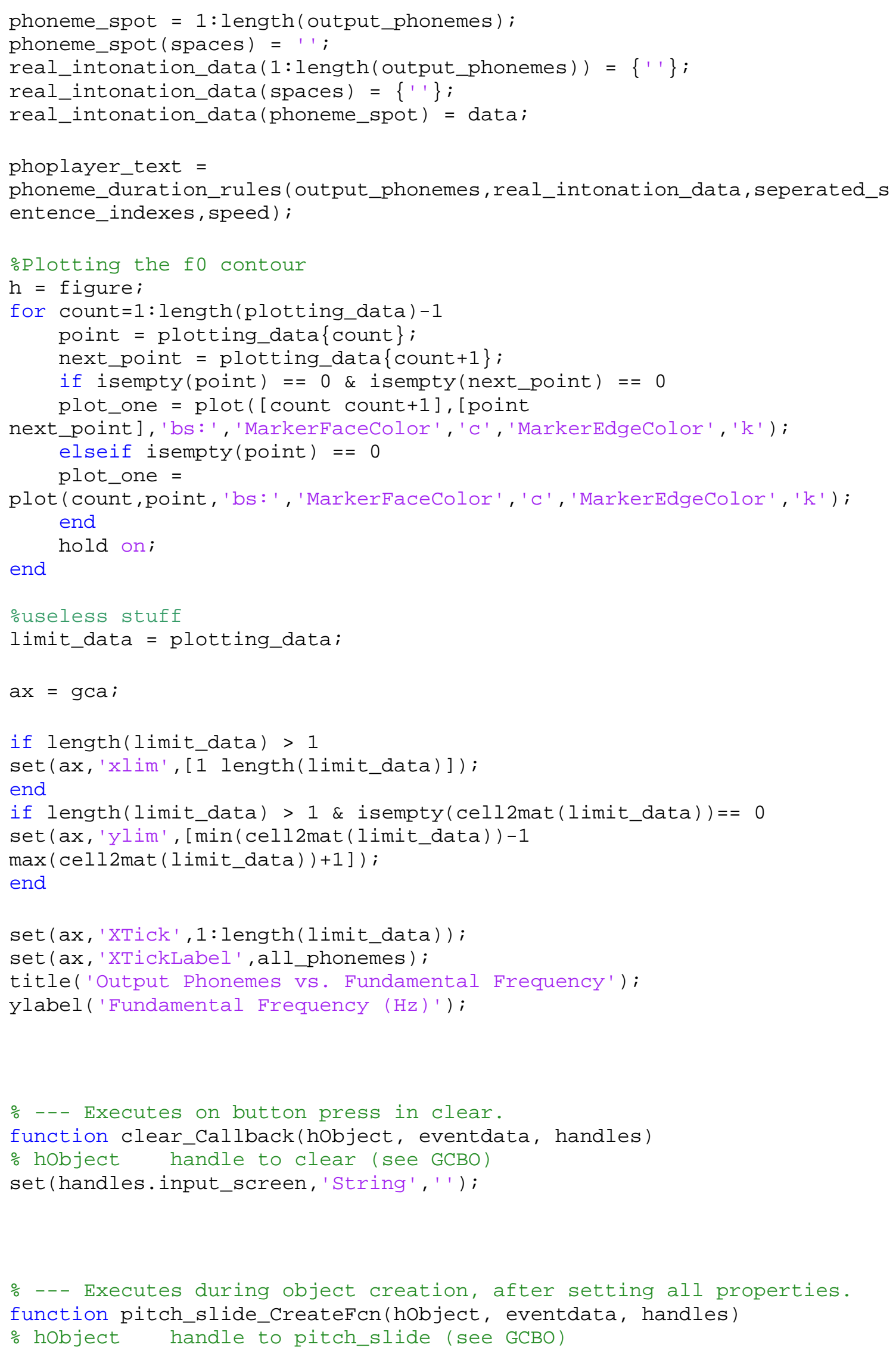




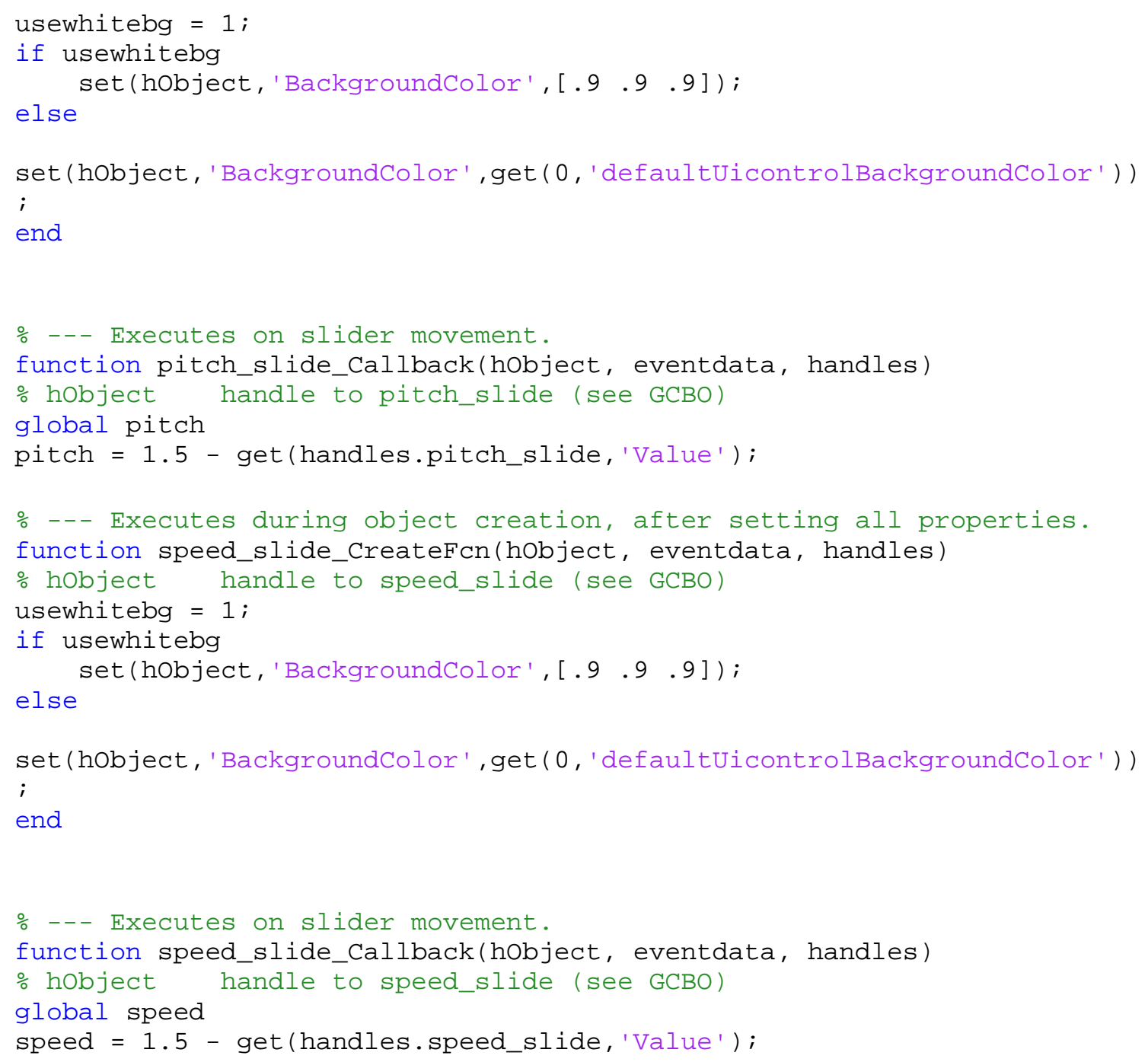




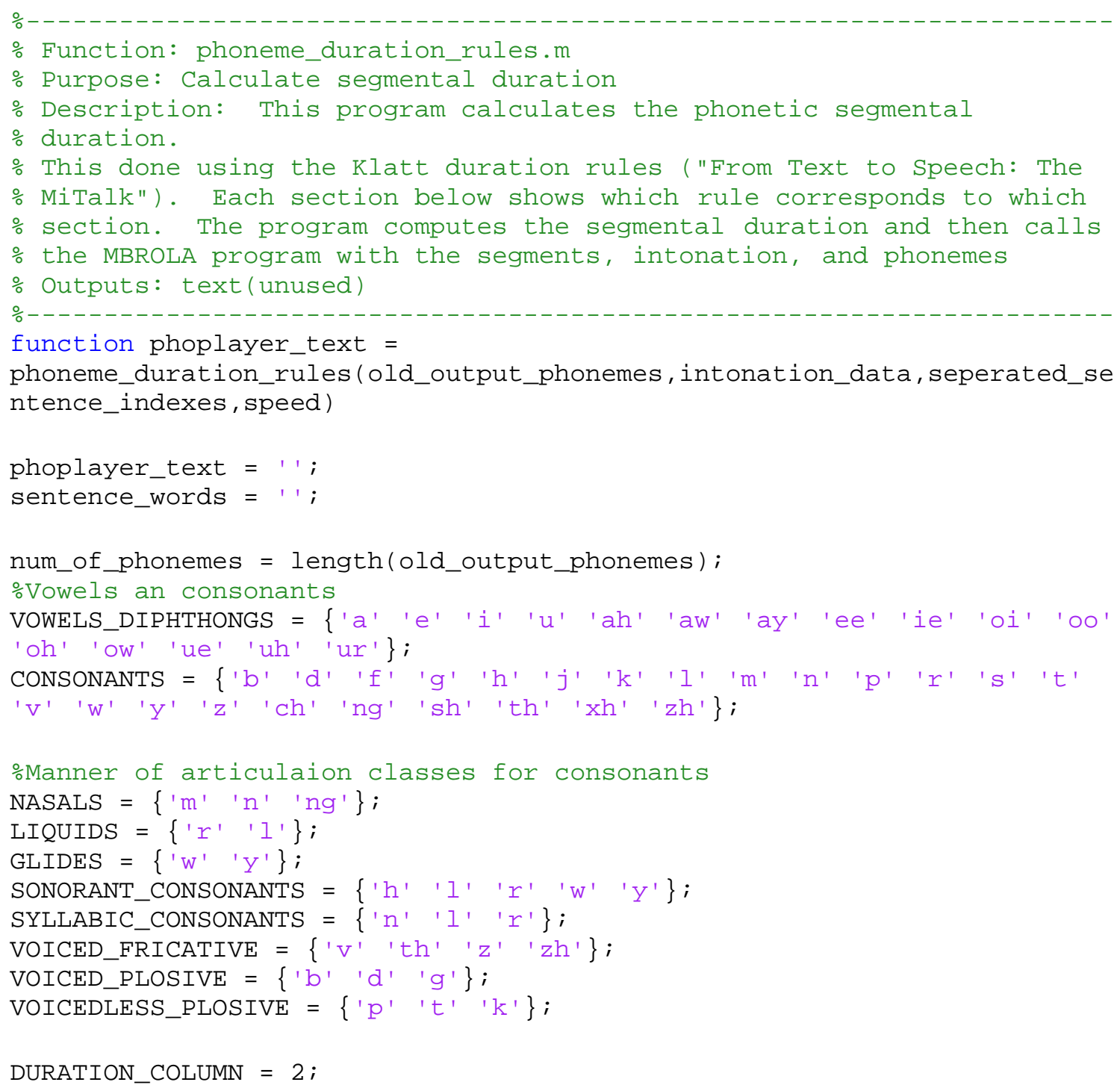

[excel_file_durations excel_file_phonemes] = xlsread('Word Data $\backslash$ Phoneme Duration.xls');

[dummy_data text_data] = xlsread('Word Data \Phoneme Convert List.xls'); phoneme_conversion_list $(:, 1)=\operatorname{text}$ _data $(:, 1)$; 


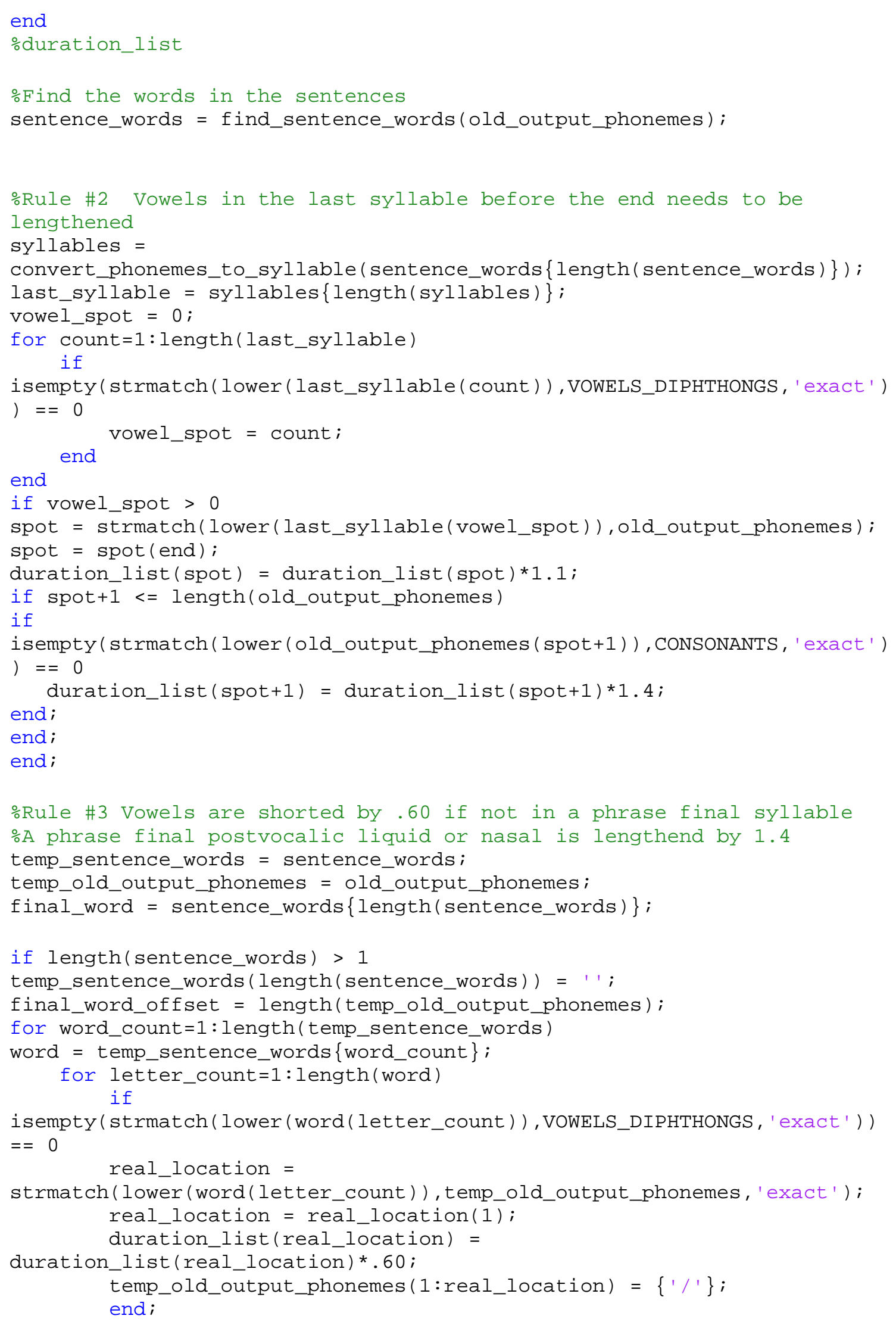




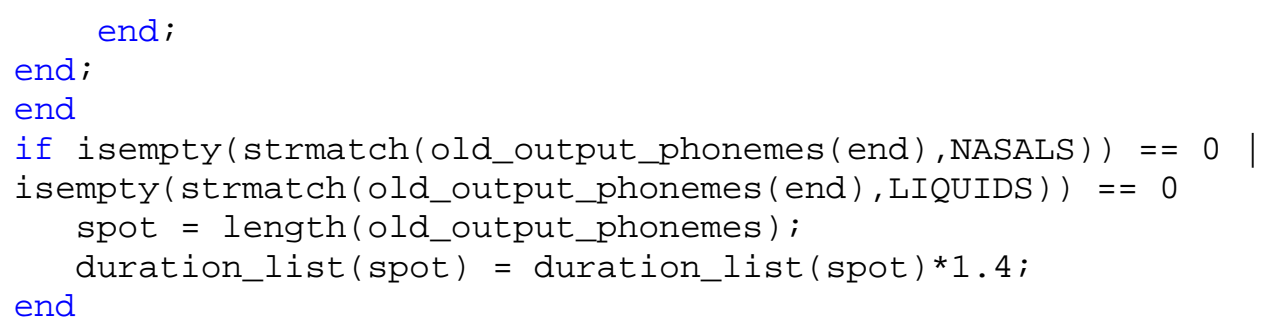




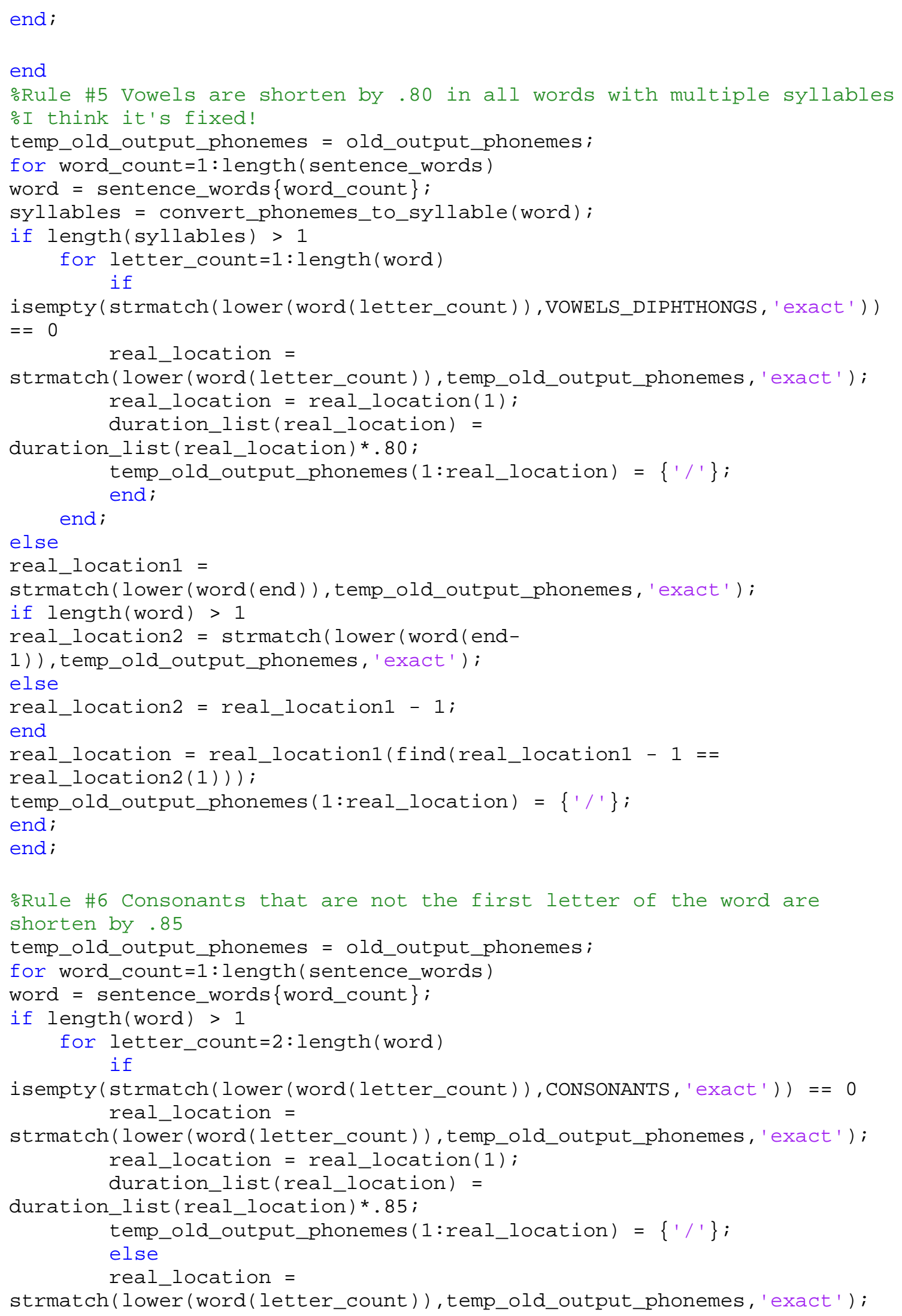




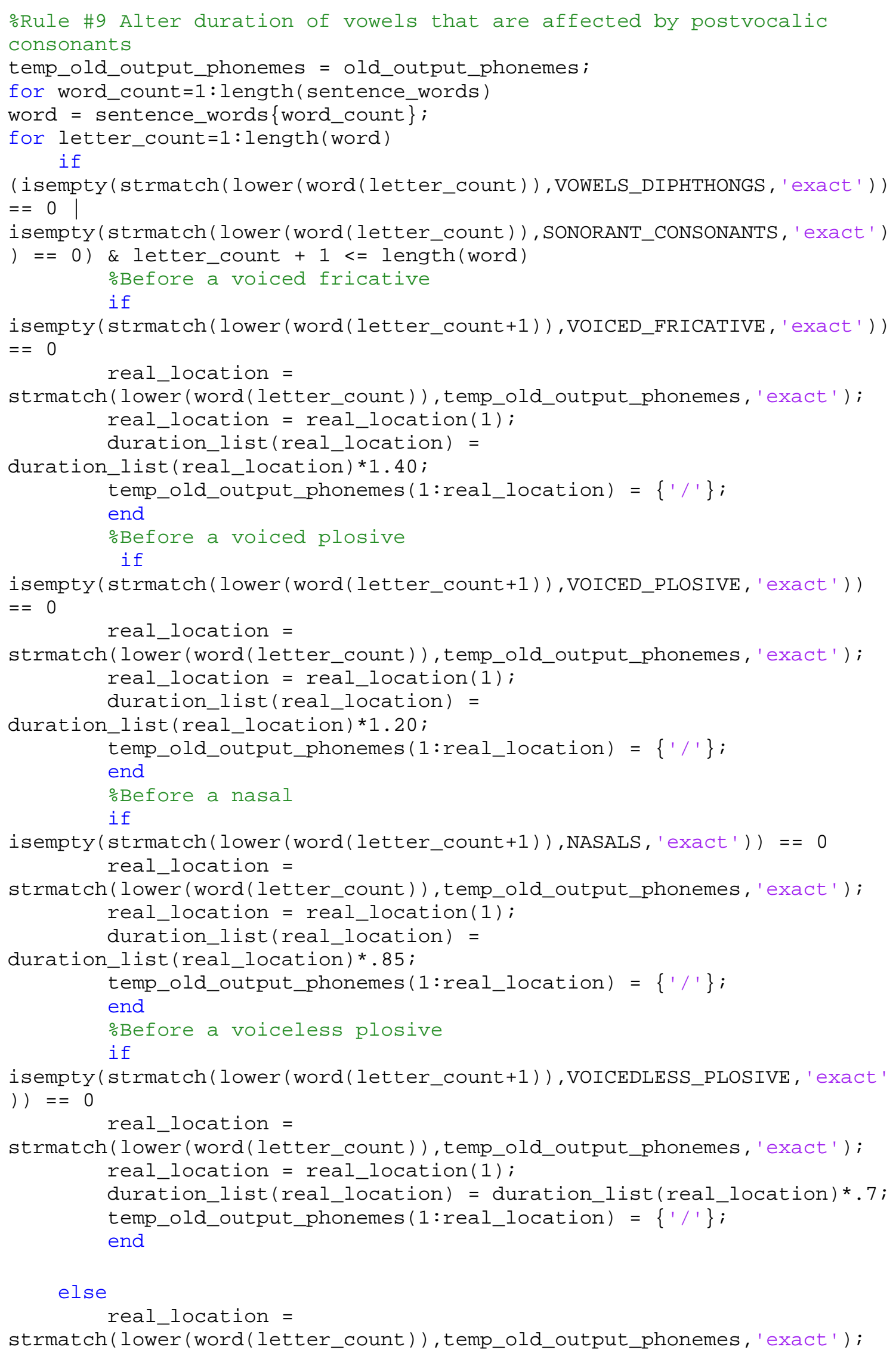


[wav_data, FS, NBITS, OPTS]= wavread( 'output.wav' );

wavplay(wav_data, FS); 


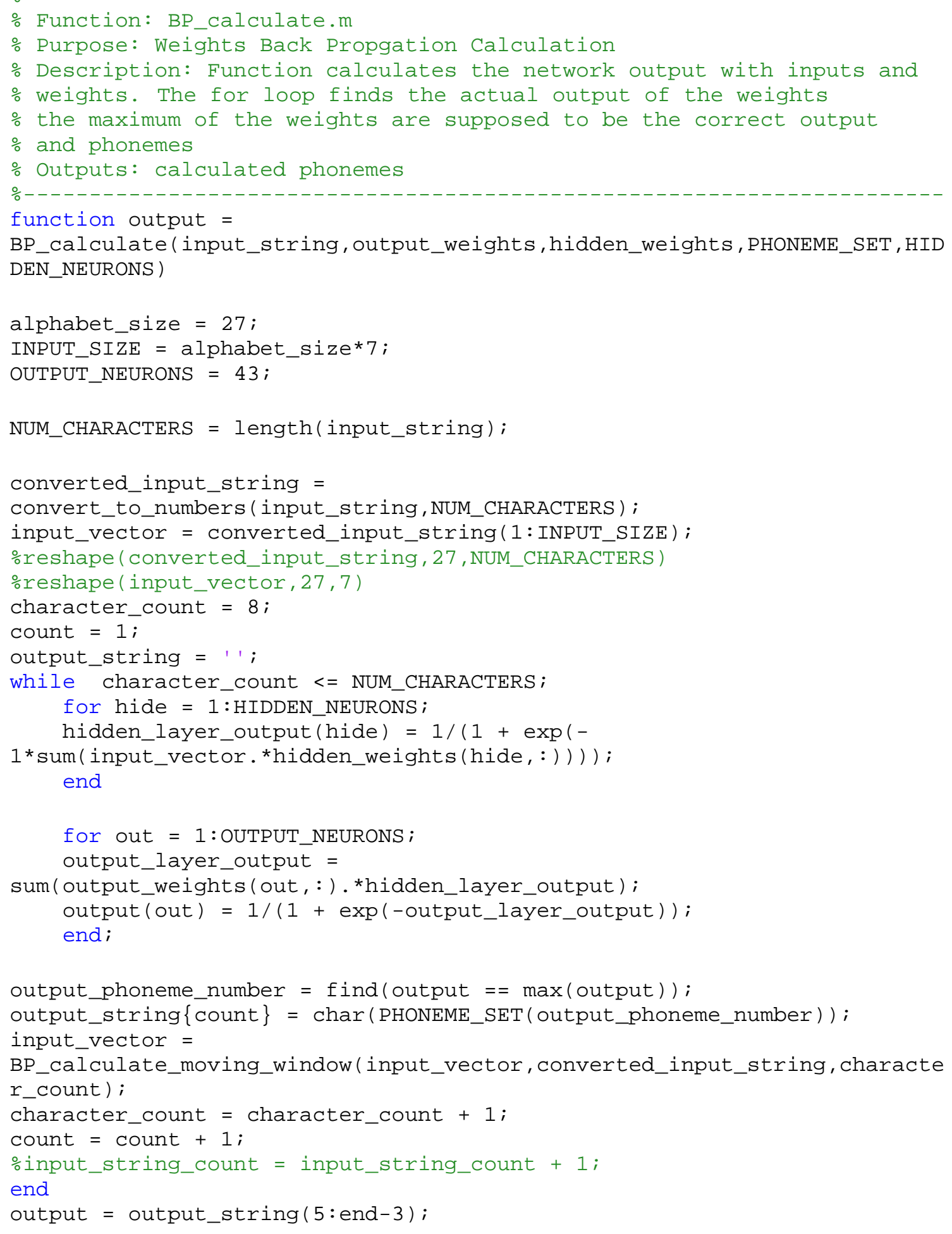




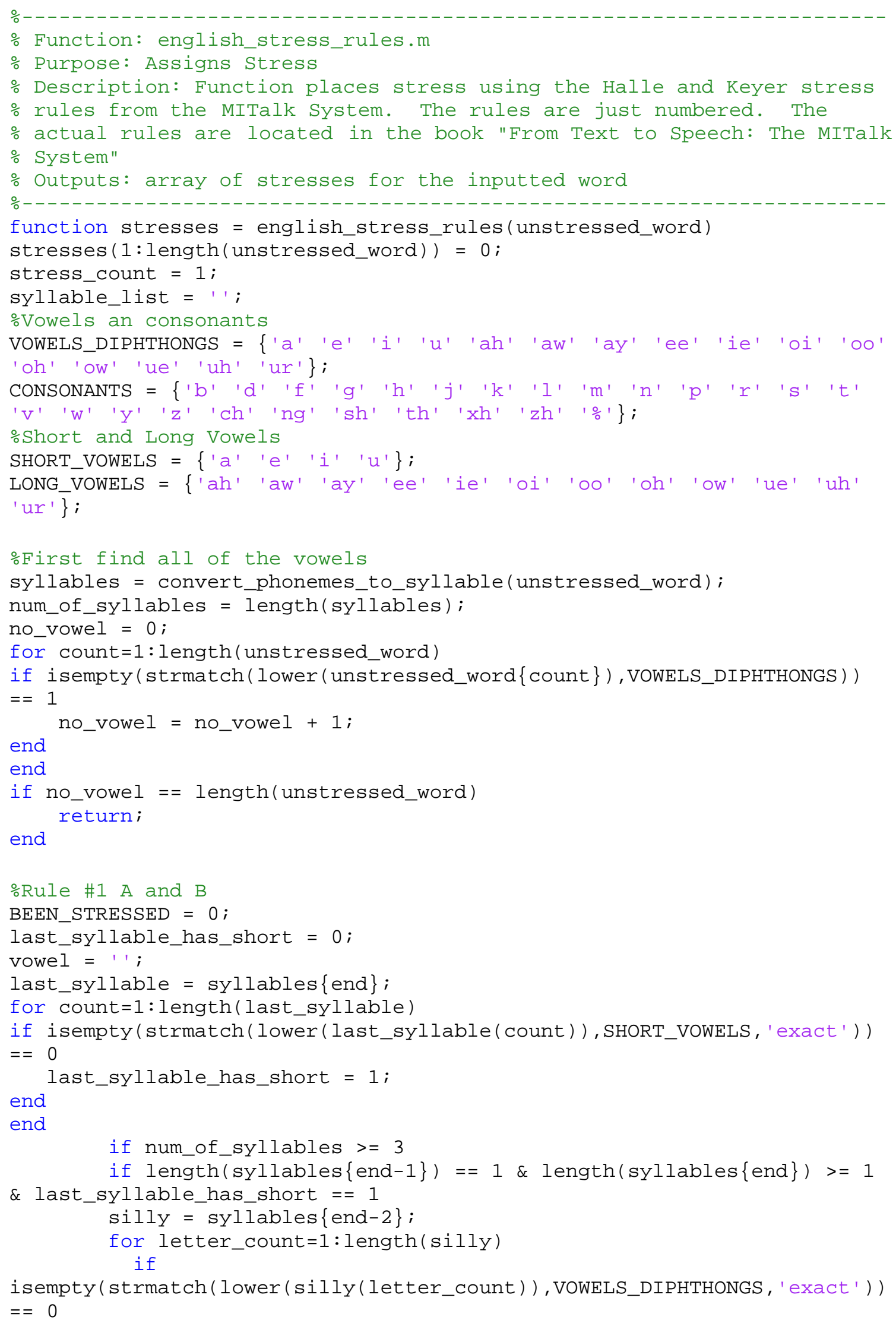




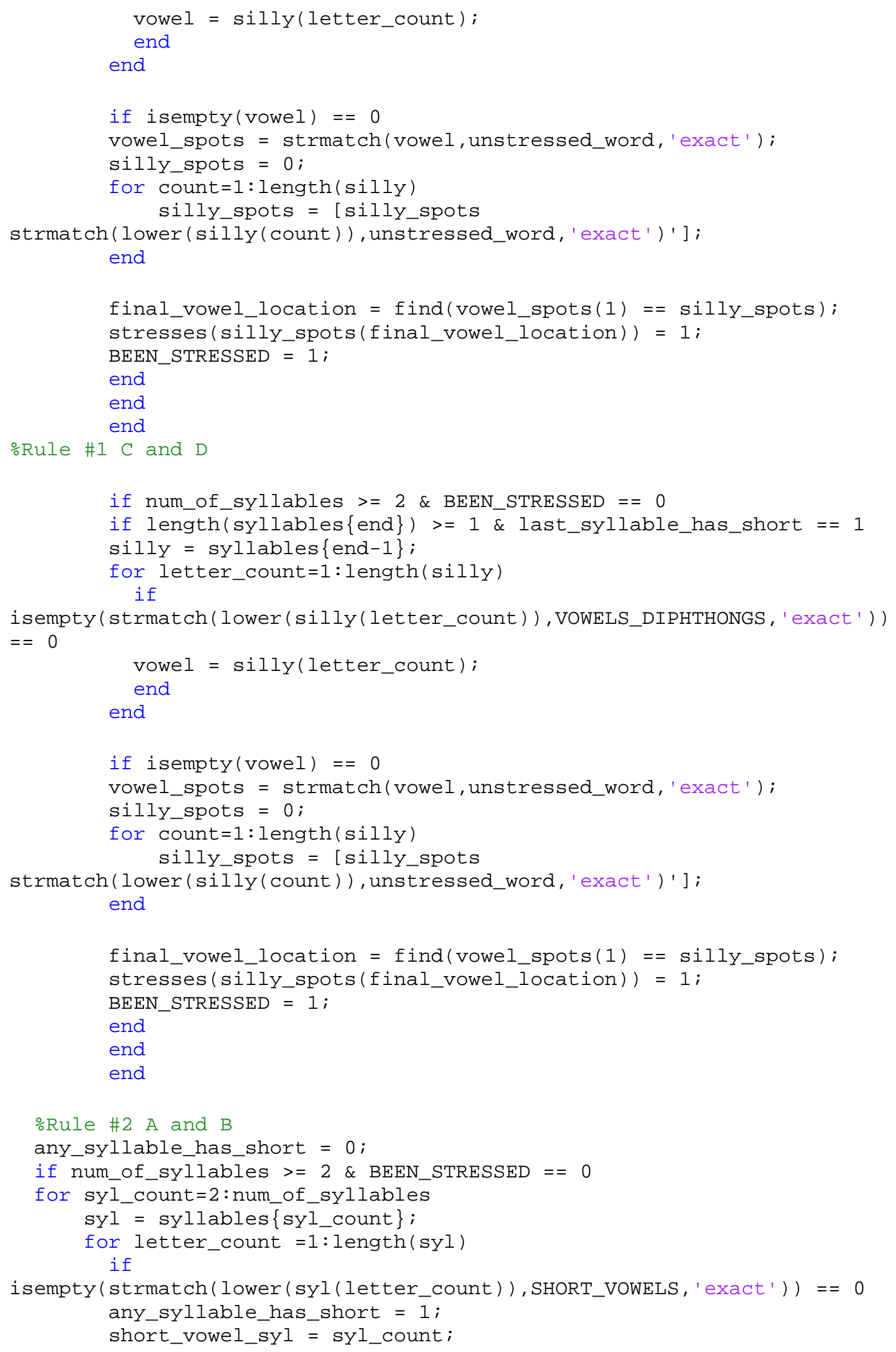




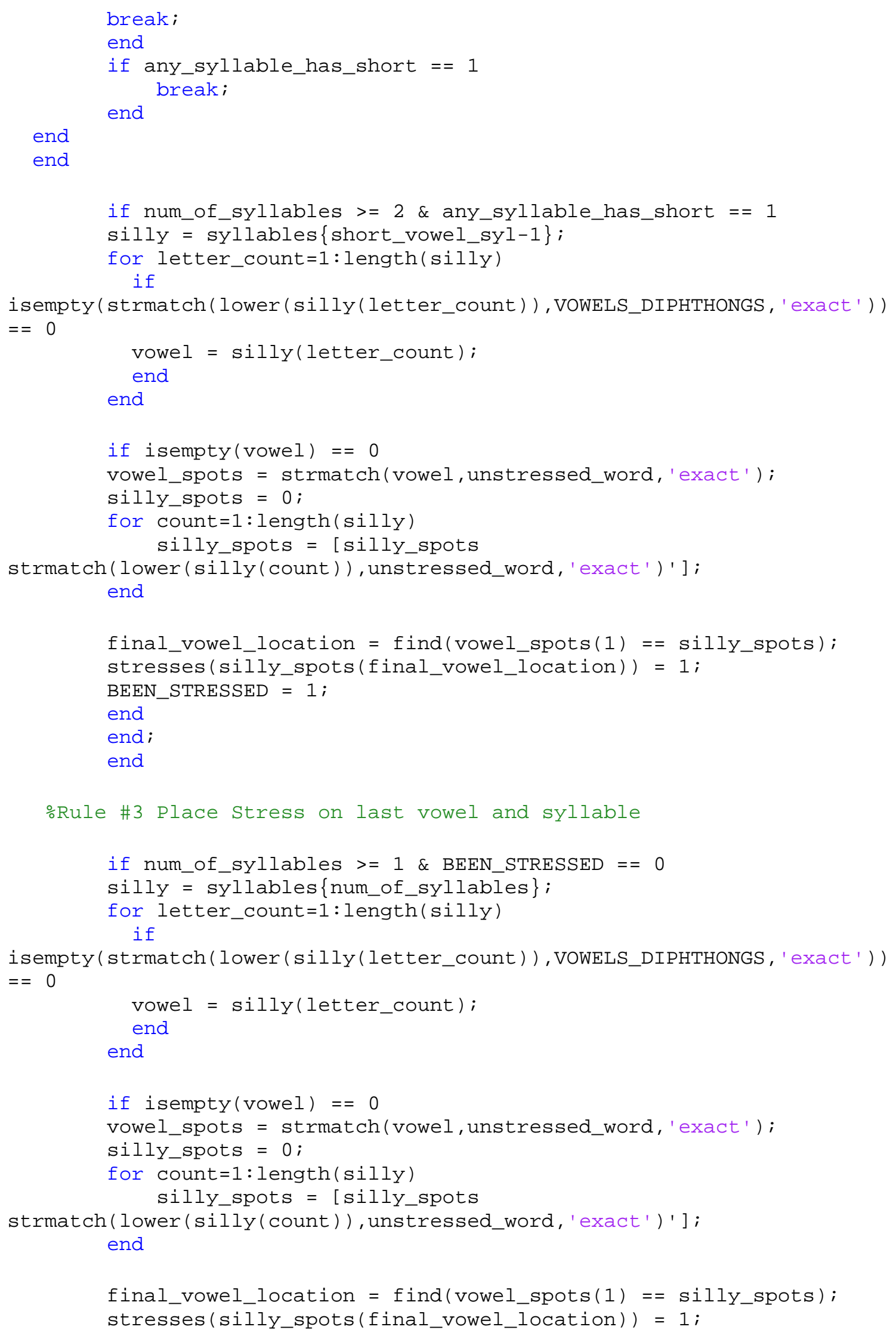




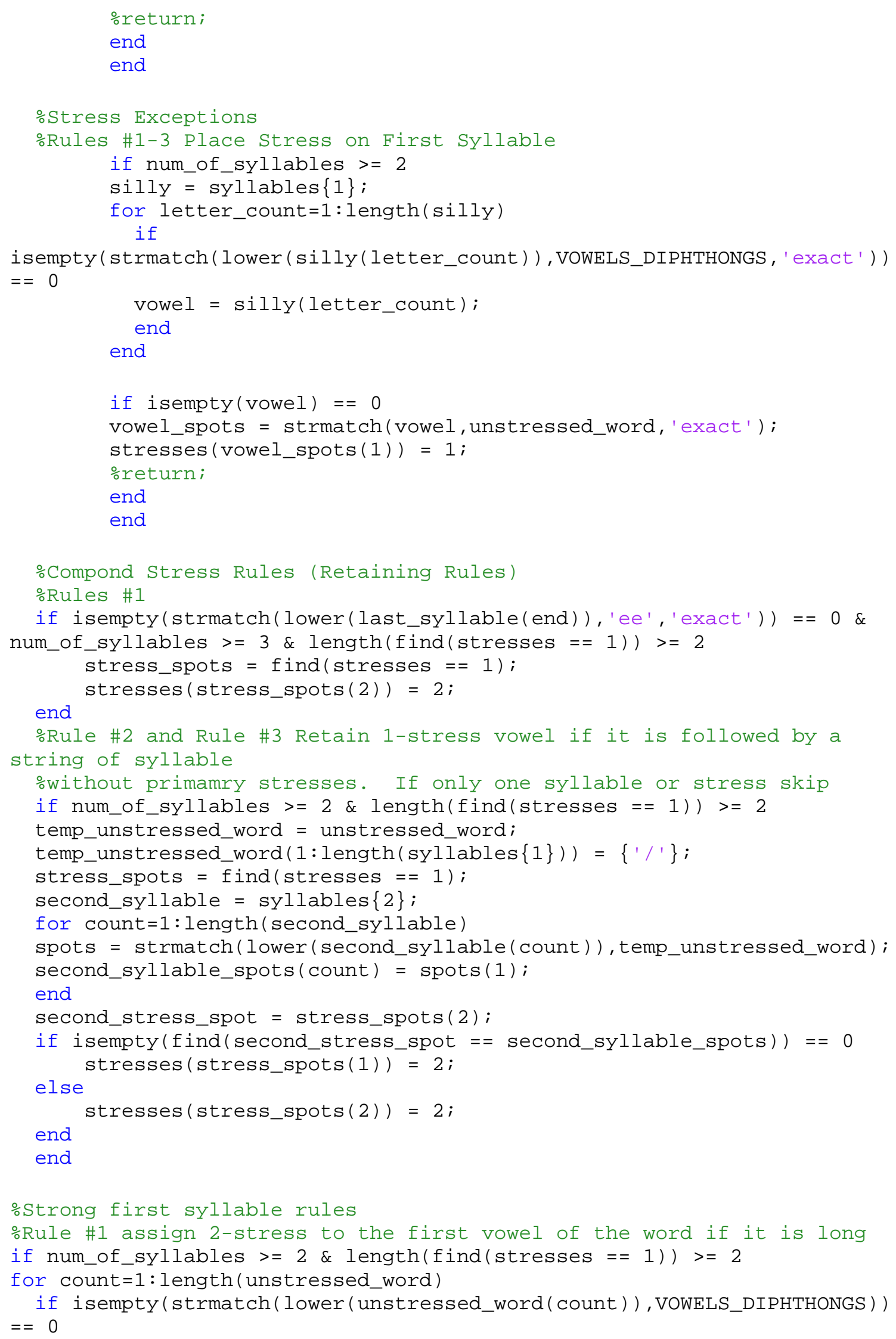




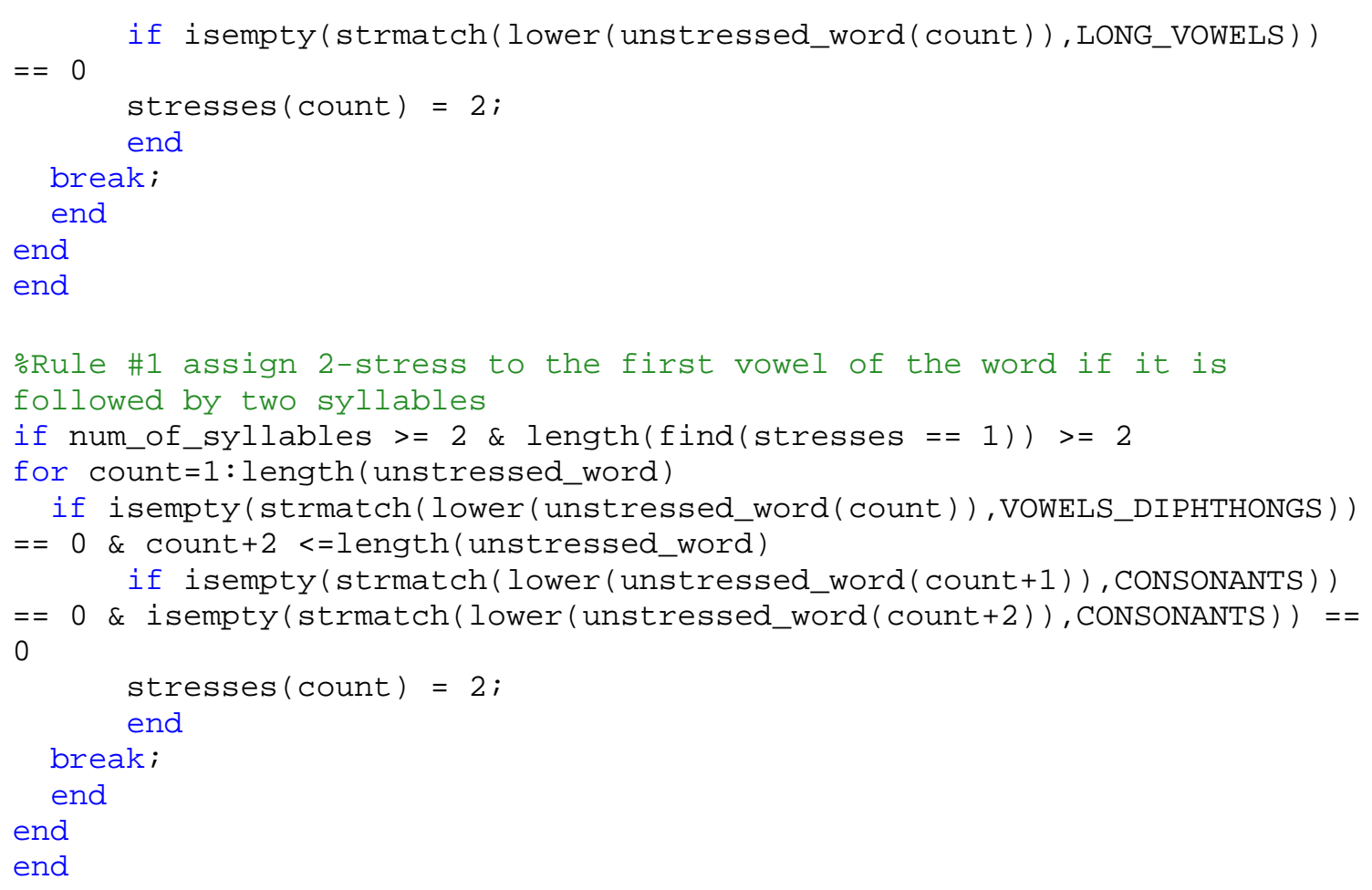




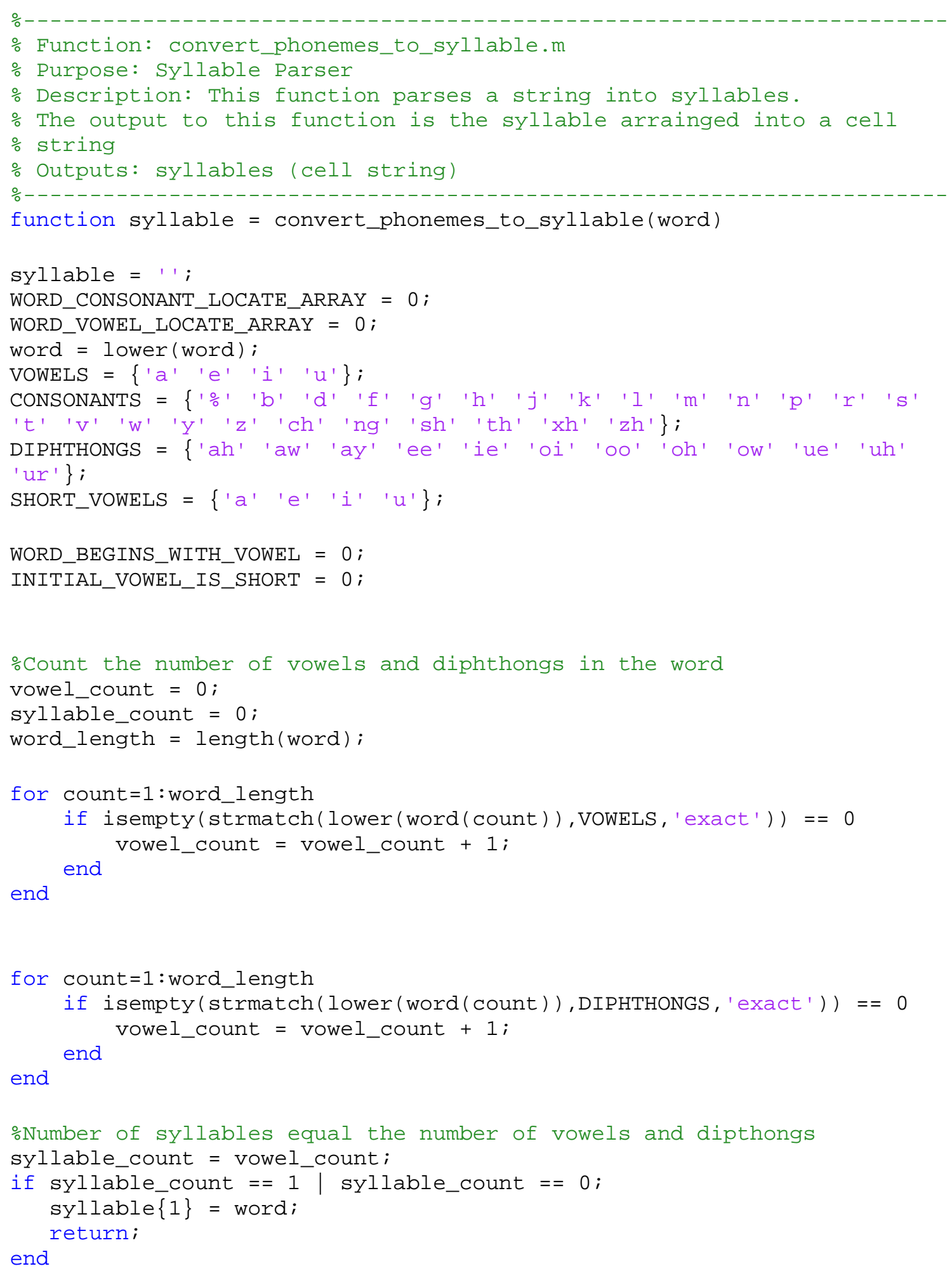




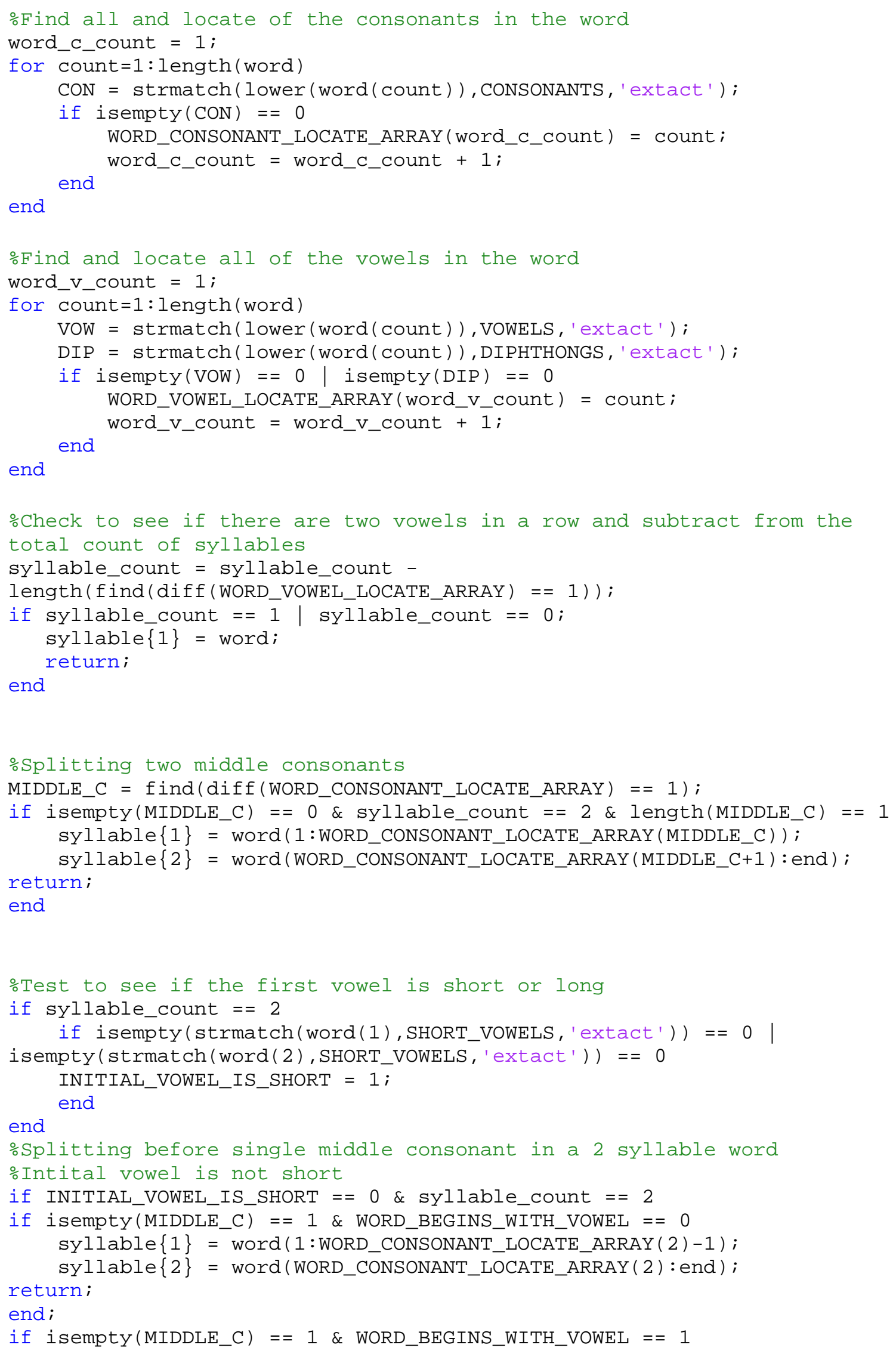




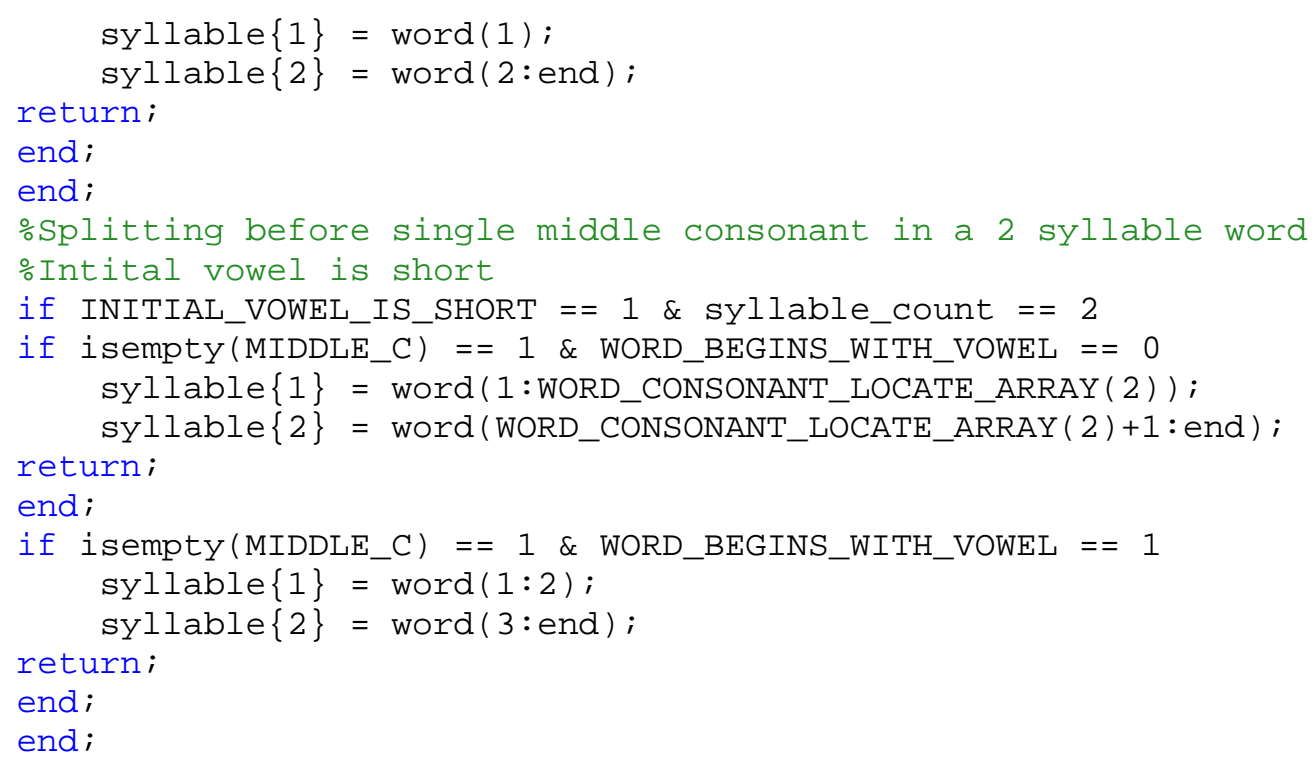


\%begins with a vowel then automatically goto this step

if isempty (find (next_letter $==$ WORD_VOWEL_LOCATE_ARRAY)) $==0 \&$

WORD_BEGINS_WITH_VOWEL $==0$

syllable = ' ';

syllable\{syl_cell_count $\}=$

word(syllable_starting_point: syllable_ending_point-1);

syllable_starting_point = syllable_ending_point;

syllable_ending_point $=$ syllable_ending_point +1 ;

else

syllable_starting_point $=$ syllable_ending_point +1 ;

end

syllable_ending_point $=$ syllable_ending_point +2 ;

end

if syl_cell_count $>1$

\%For the rest of rest of the iterations, check to see if the second

\%letter after the lat letter in the 2 letter syllable is a vowel

\%If so, then update the syllable location for the next syllable

\%If the letter is a consonant then make the syllable 3 letters long

\%and update the syllable location for the next syllable

if isempty (find (next_next_letter $==$ WORD_VOWEL_LOCATE_ARRAY) $)=0$

syllable_starting_point $=$ syllable_ending_point +1 ;

else

syllable_ending_point $=$ syllable_ending_point +2 ;

if syllable_starting_point < length(word) \&

syllable_ending_point $+1<=$ length(word)

syllable\{syl_cell_count $\}=$

word(syllable_starting_point: syllable_ending_point+1);

syllable_starting_point $=$ syllable_ending_point +2 ;

syllable_ending_point $=$ syllable_ending_point +3 ;

if syllable_ending_point > length(word)

syllable_starting_point = syllable_starting_point - 1;

syllable_ending_point = syllable_ending_point - 1;

syllable\{syl_cell_count $\}=$

word(syllable_starting_point: syllable_ending_point-1);

end

end

end

end

\%for loop

end

output_length $=0$;

syllable_start $=$ ' ';

for count $=1$ : length (syllable)

output_length = output_length + length (syllable\{count $\})$;

syllable_start $\{$ count $\}=$ output_length - length(syllable $\{$ count $\}) ;$

end 
if output_length < word_length

temp_word $=$ word $($ syllable_start $\{$ end $\}+1$ : word_length $)$;

syllable $\{$ end $\}=$ temp_word;

end

\%syllable_count $>2$

end

\%END OF PROGRAM 


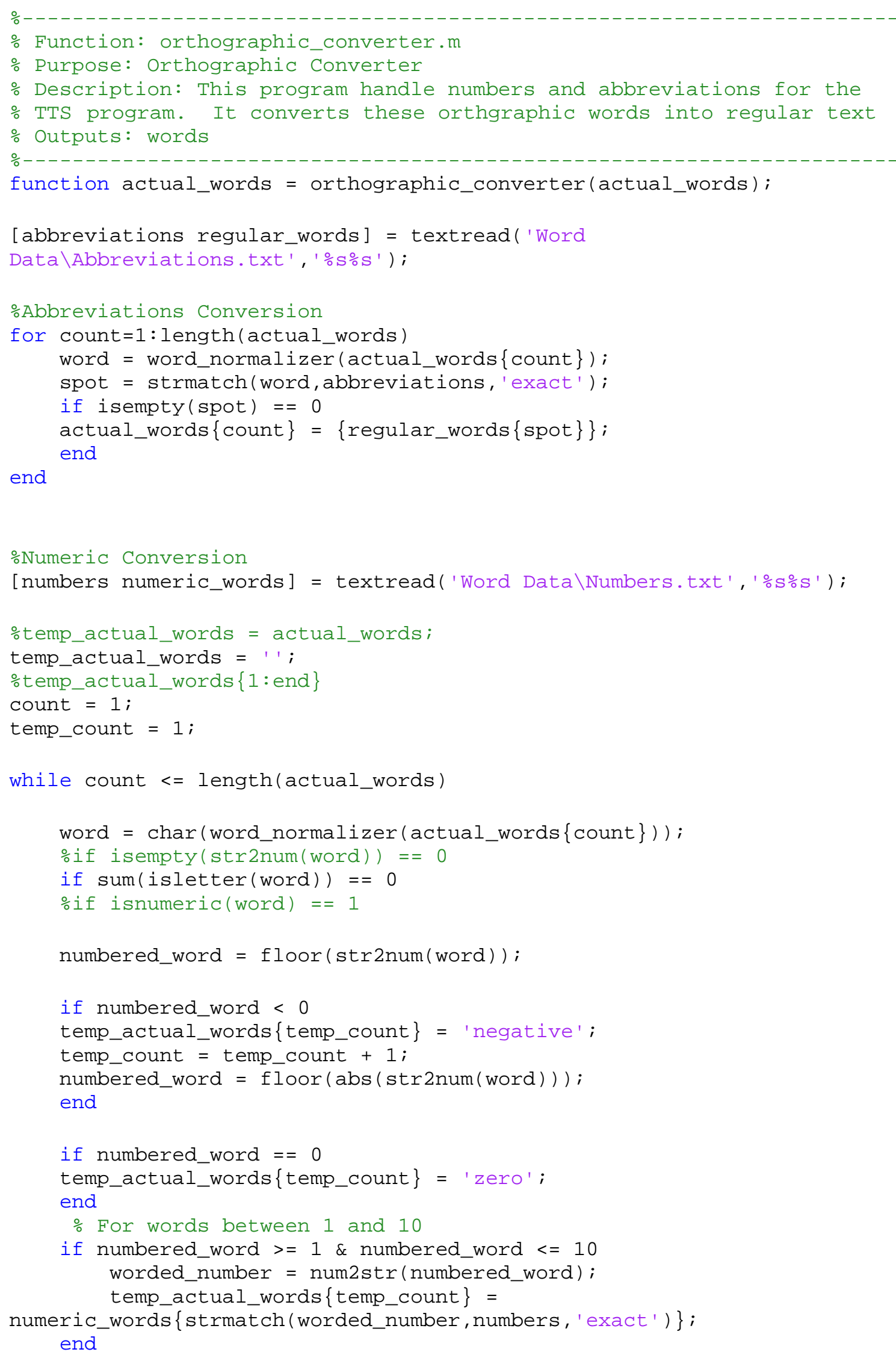




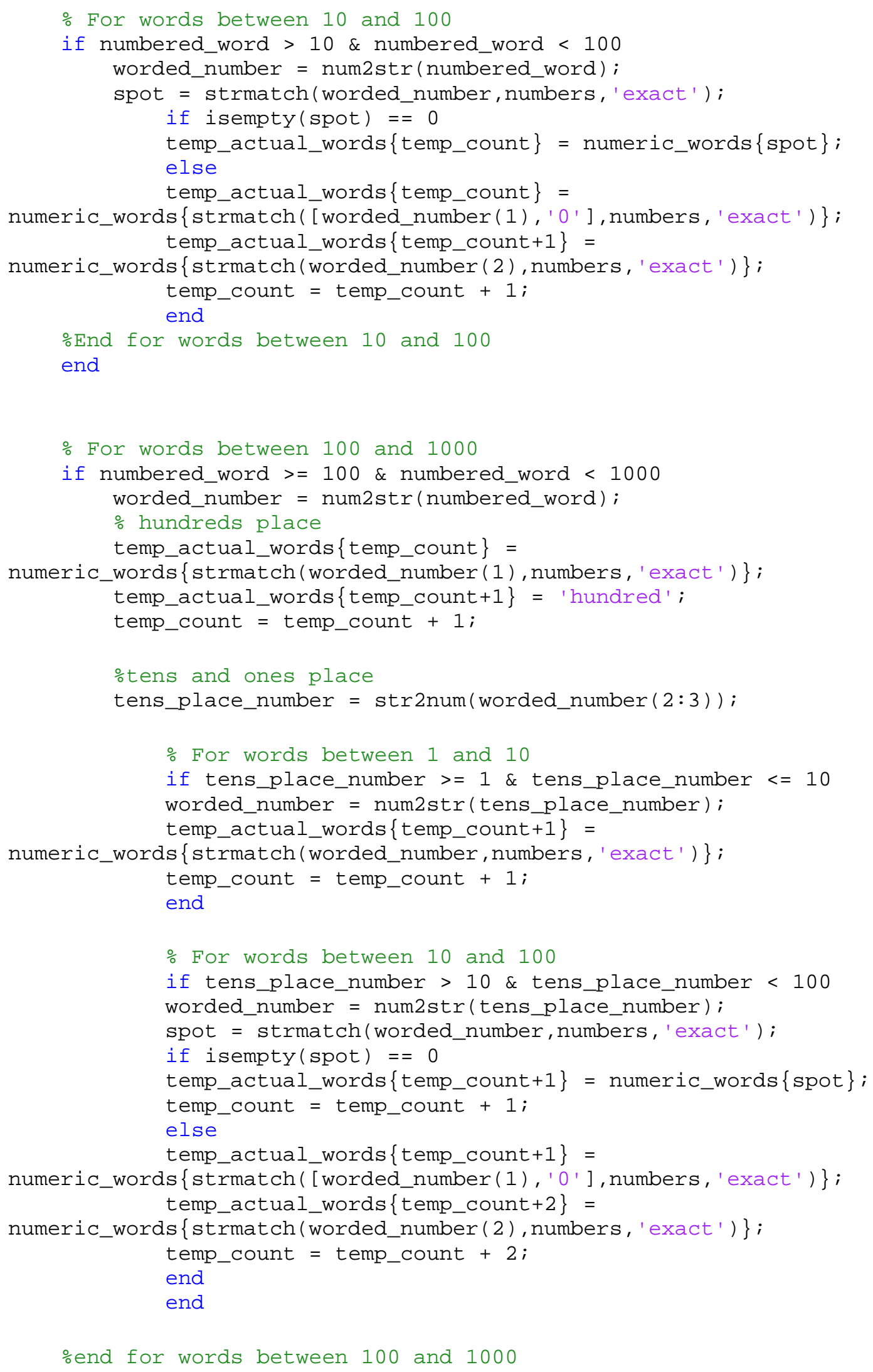




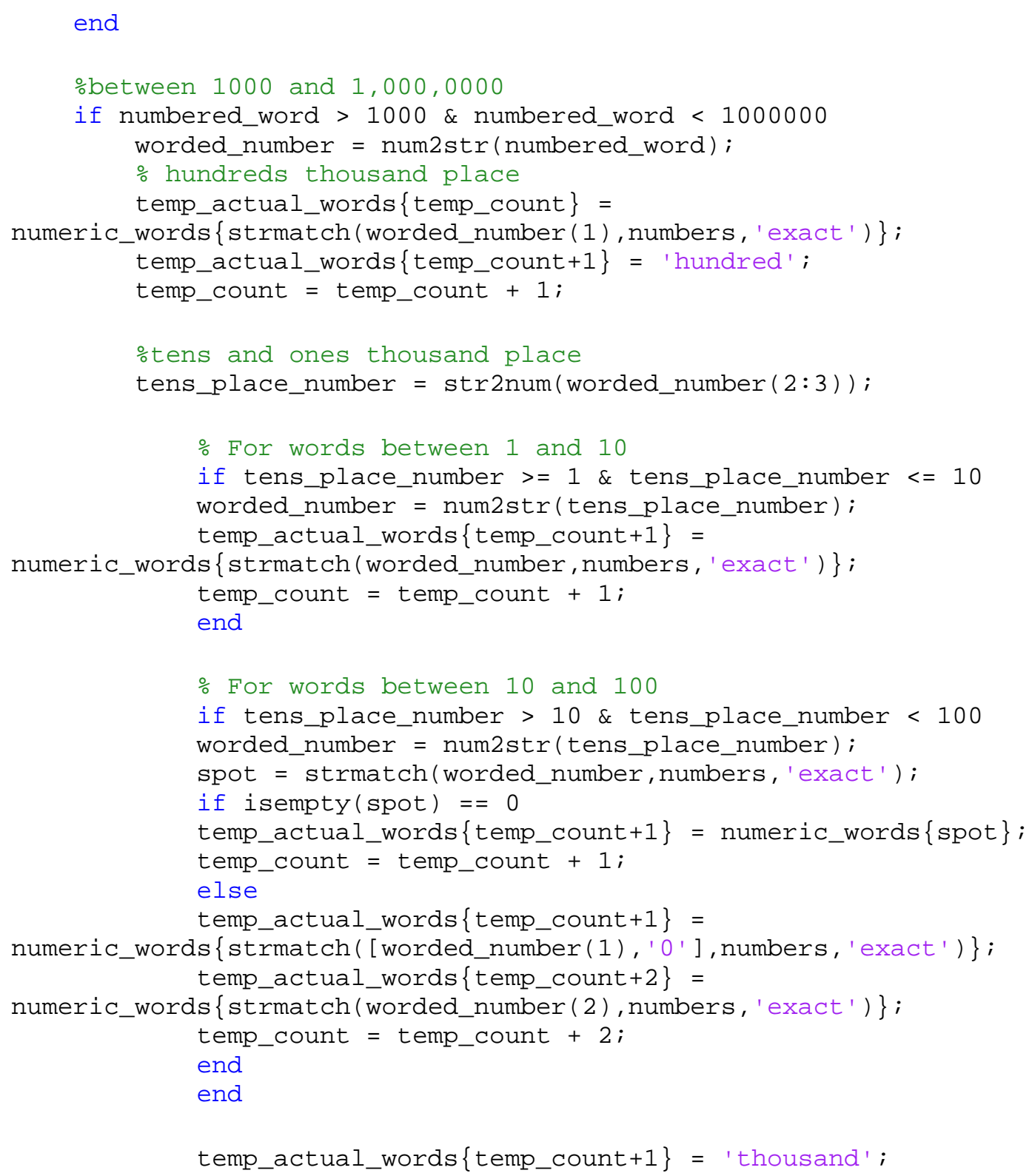




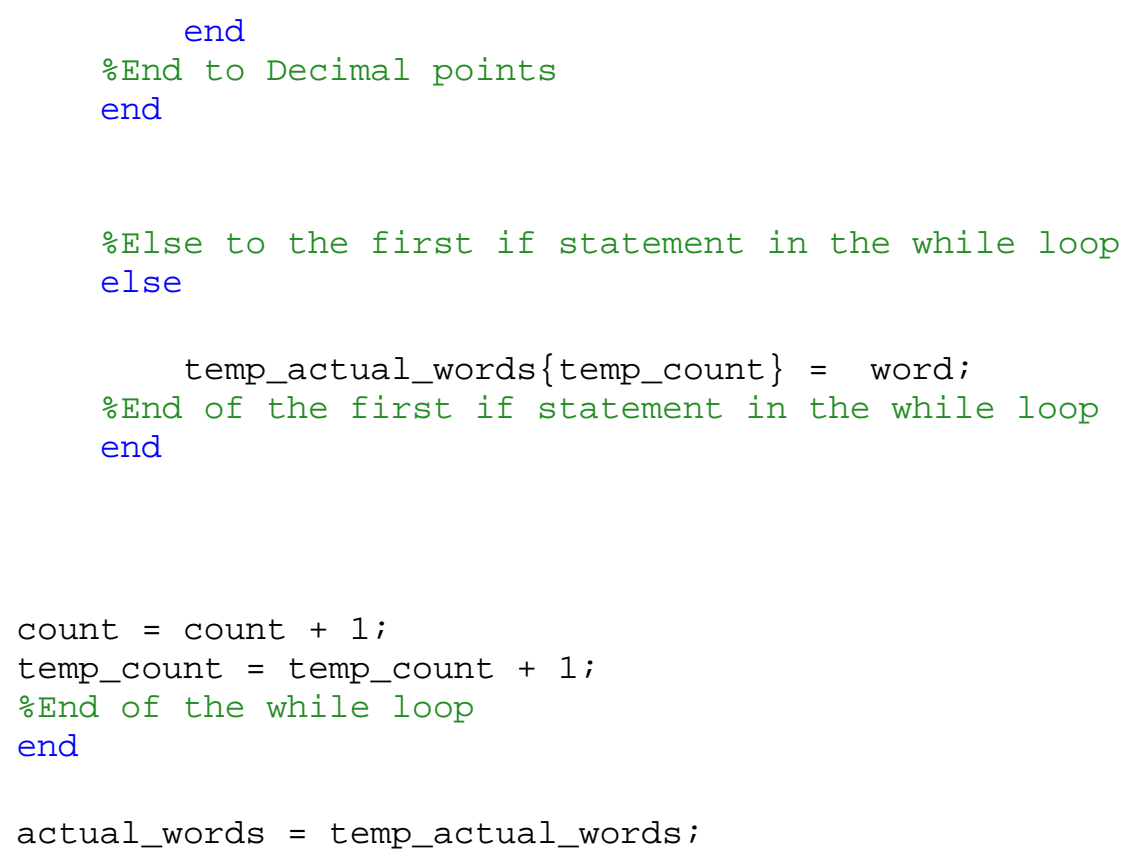




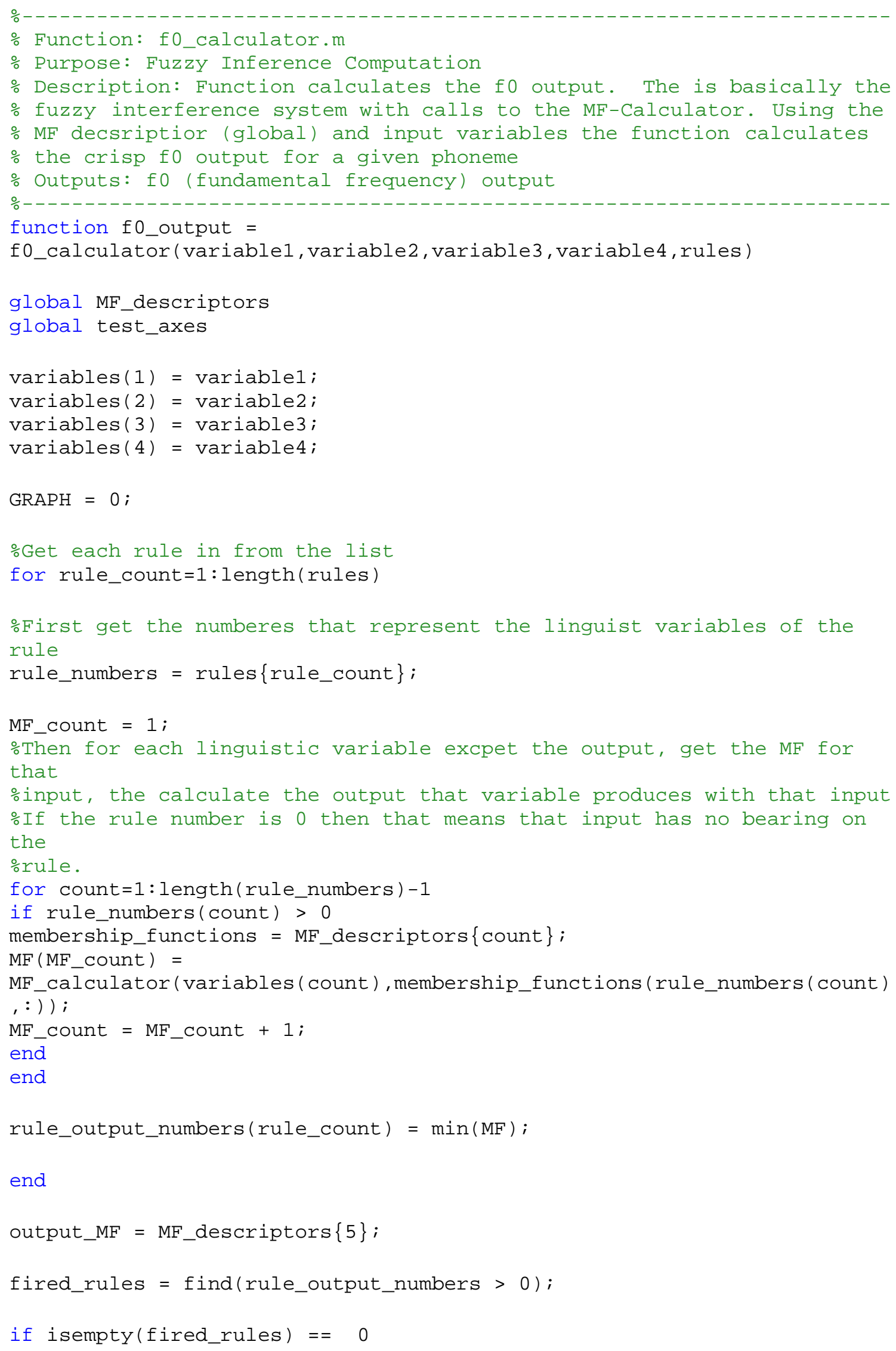




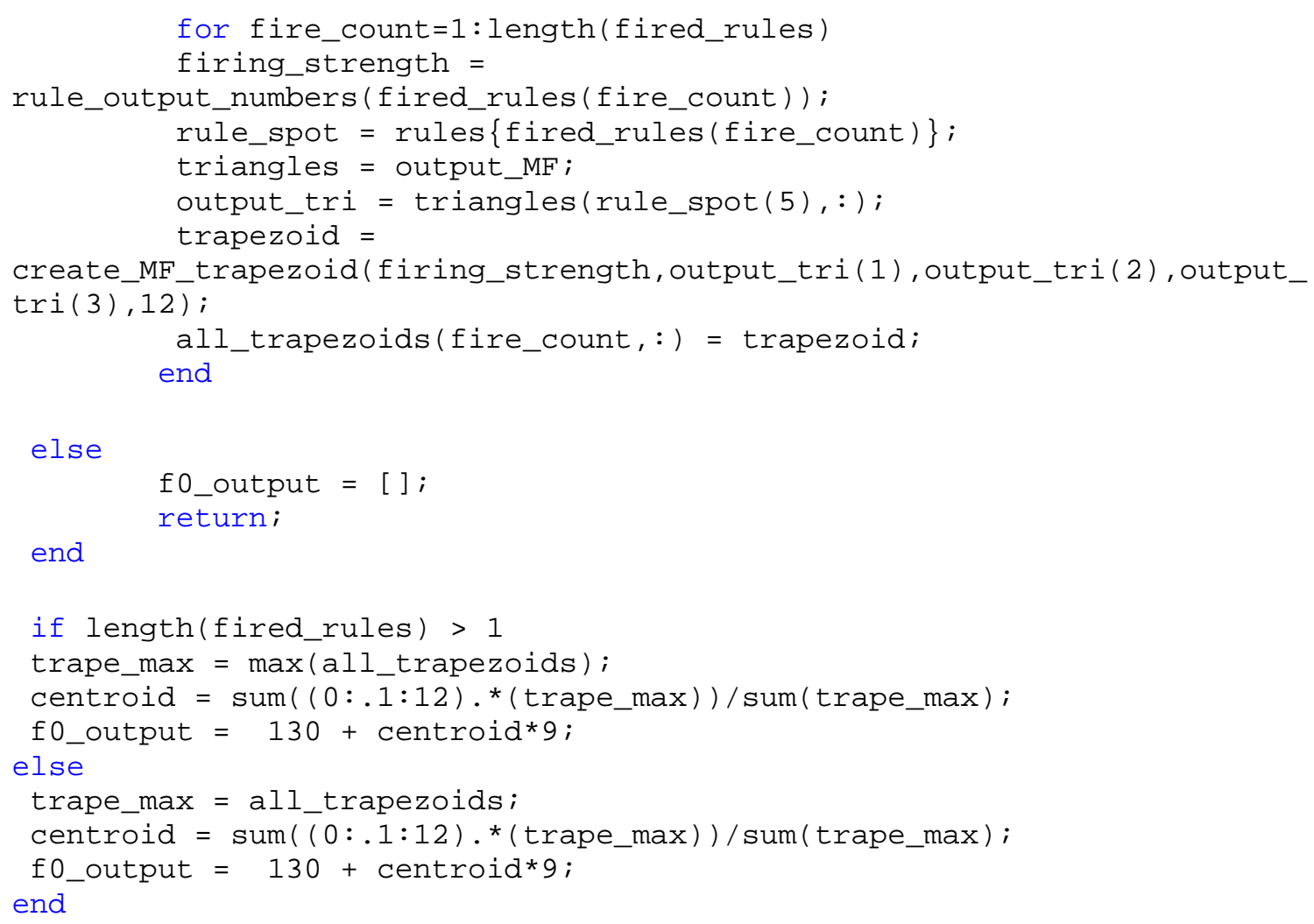




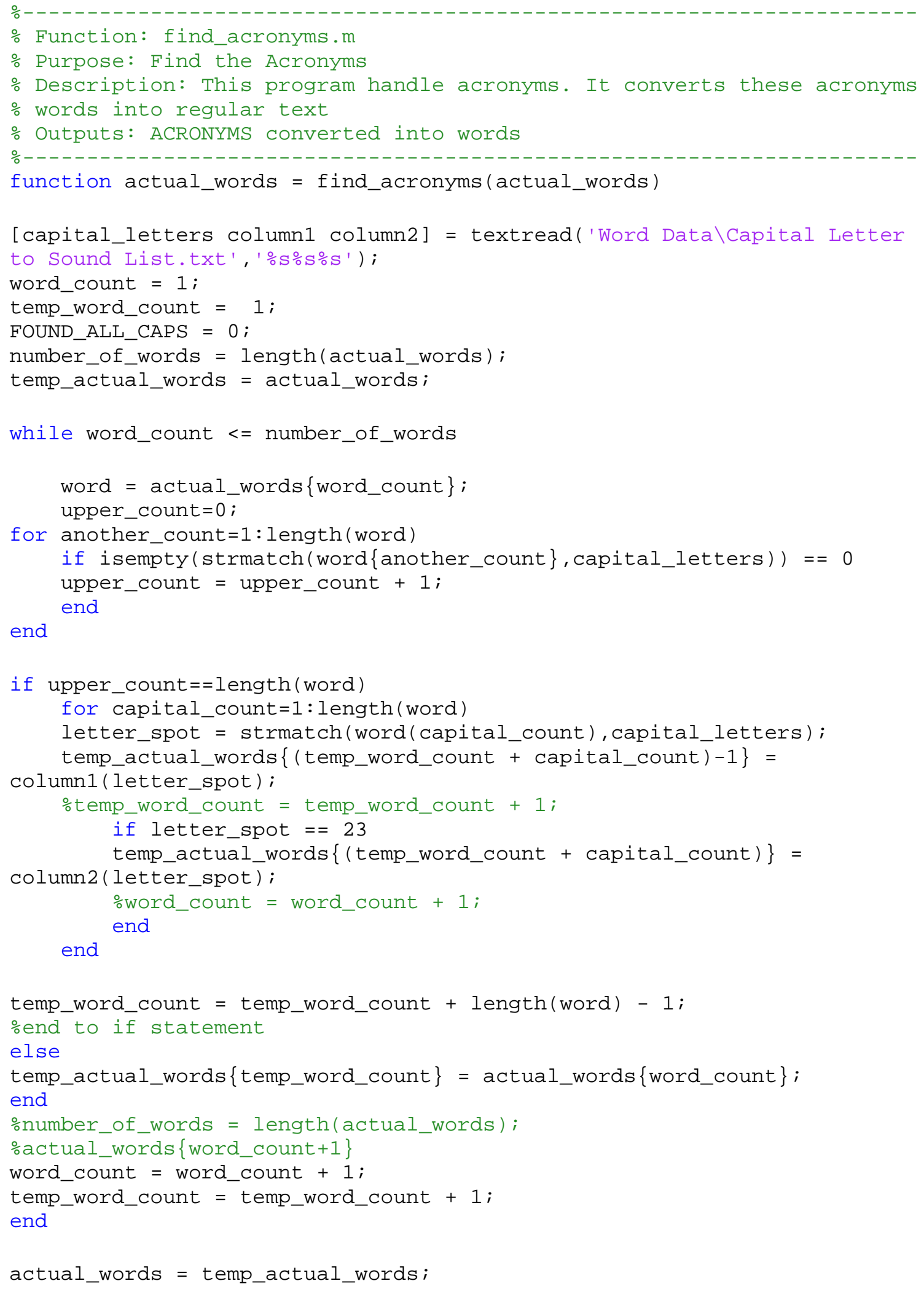




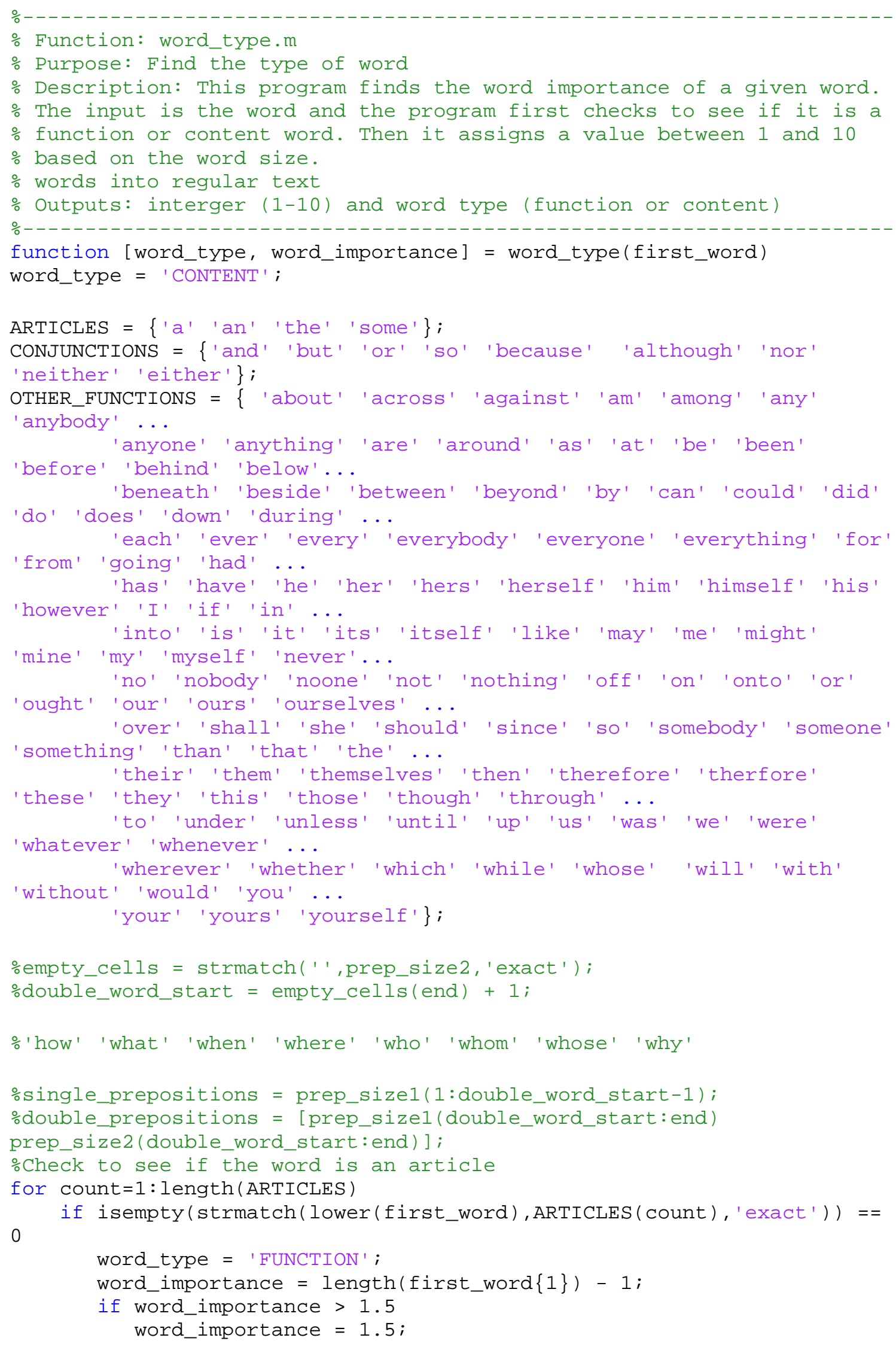




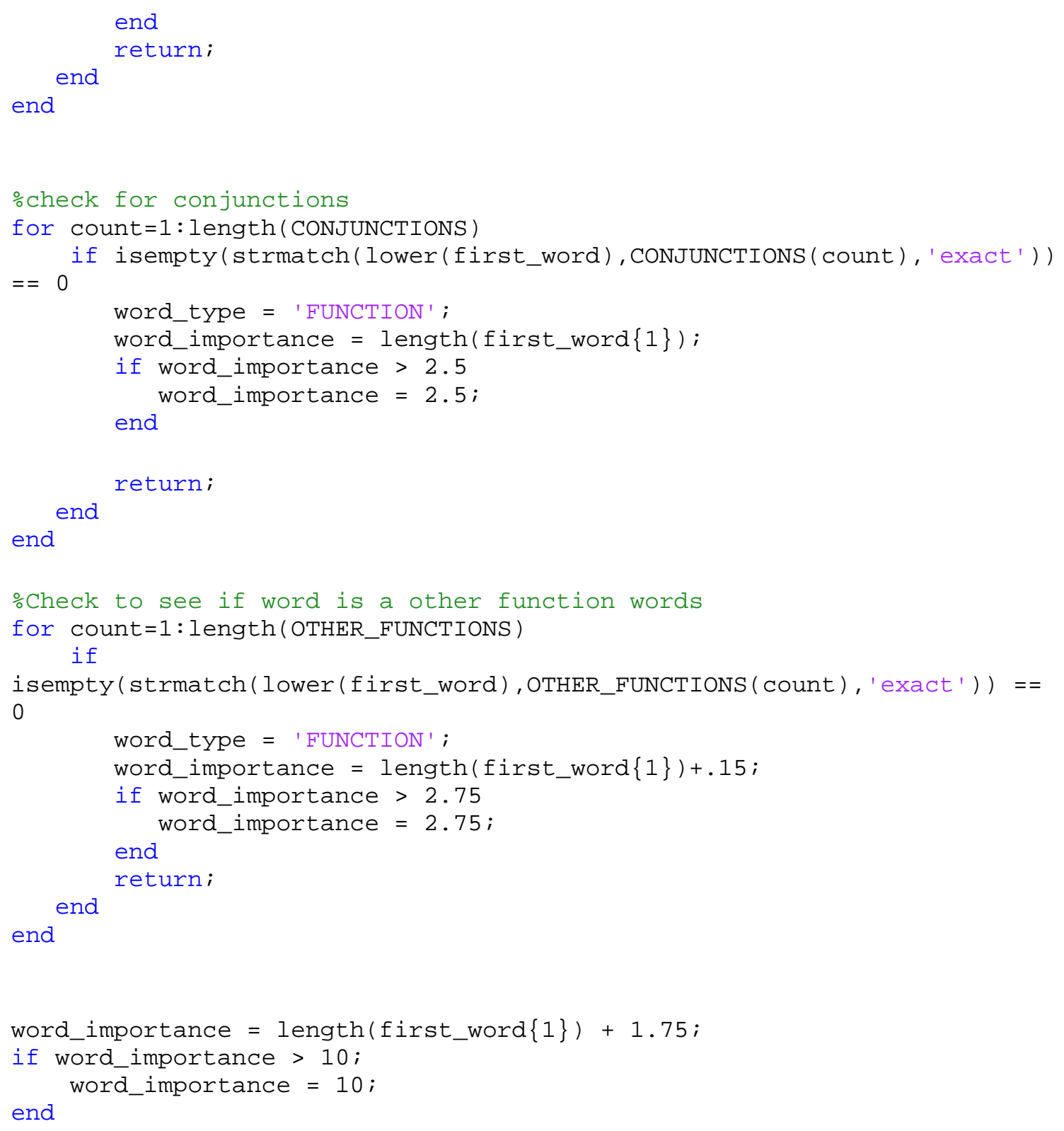




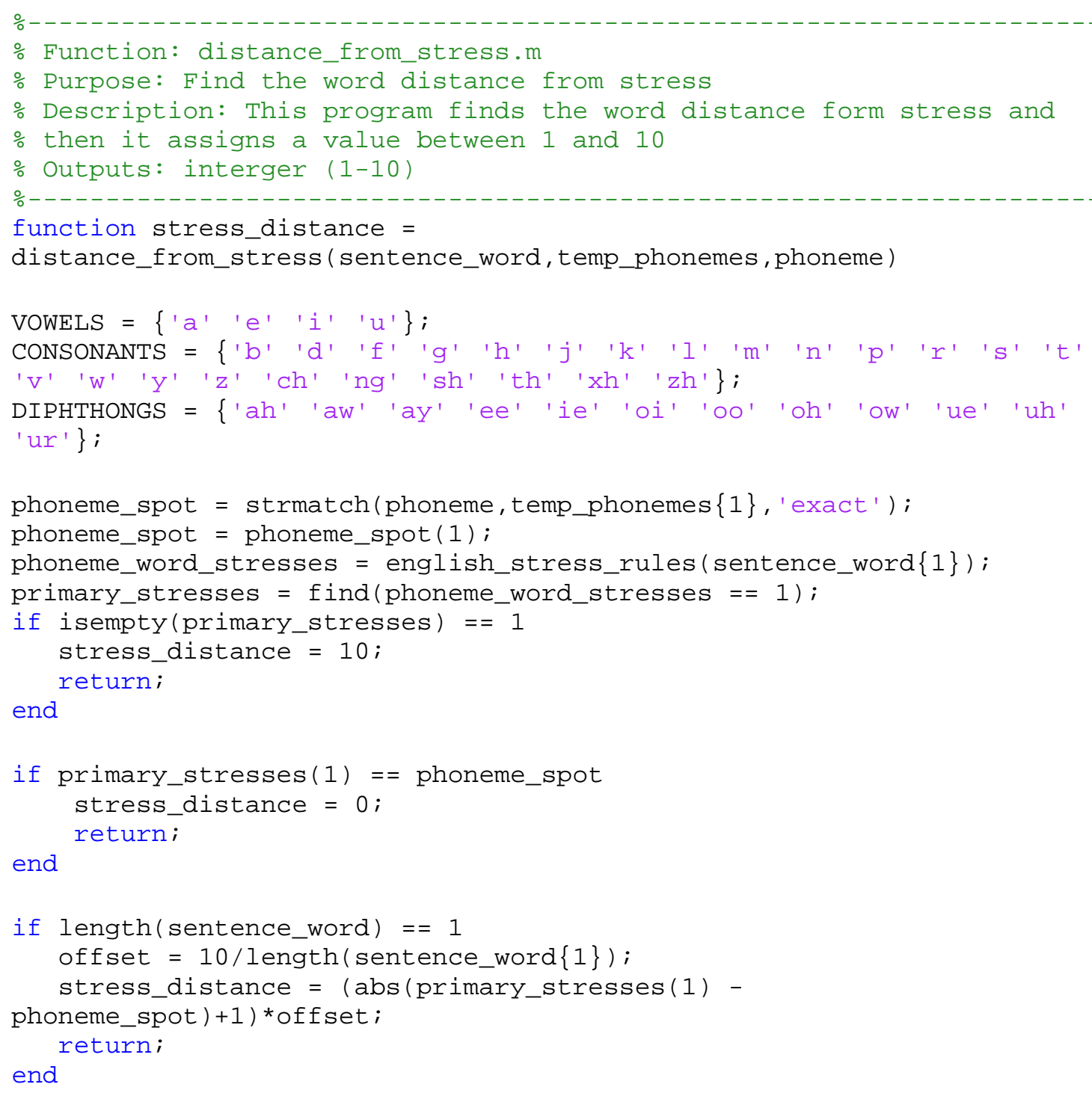




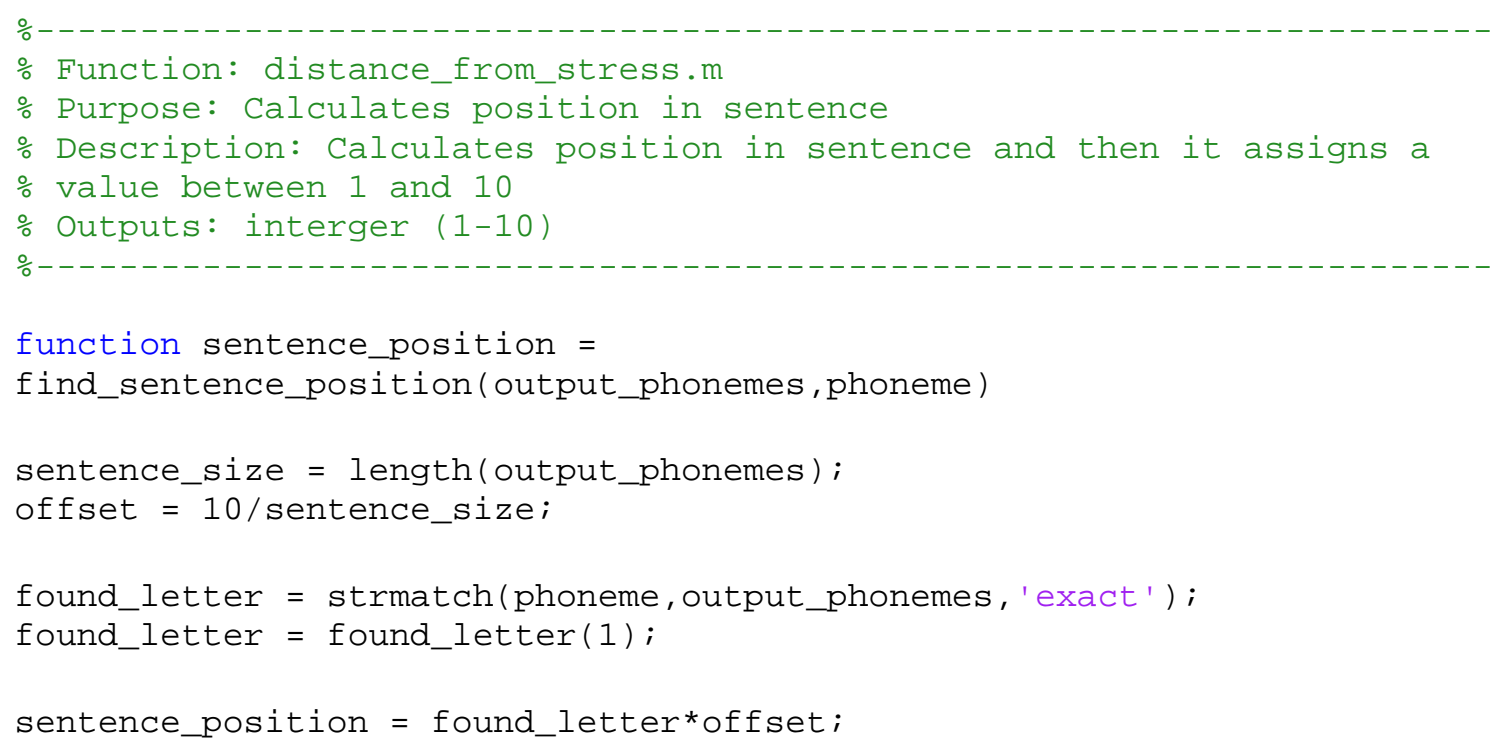




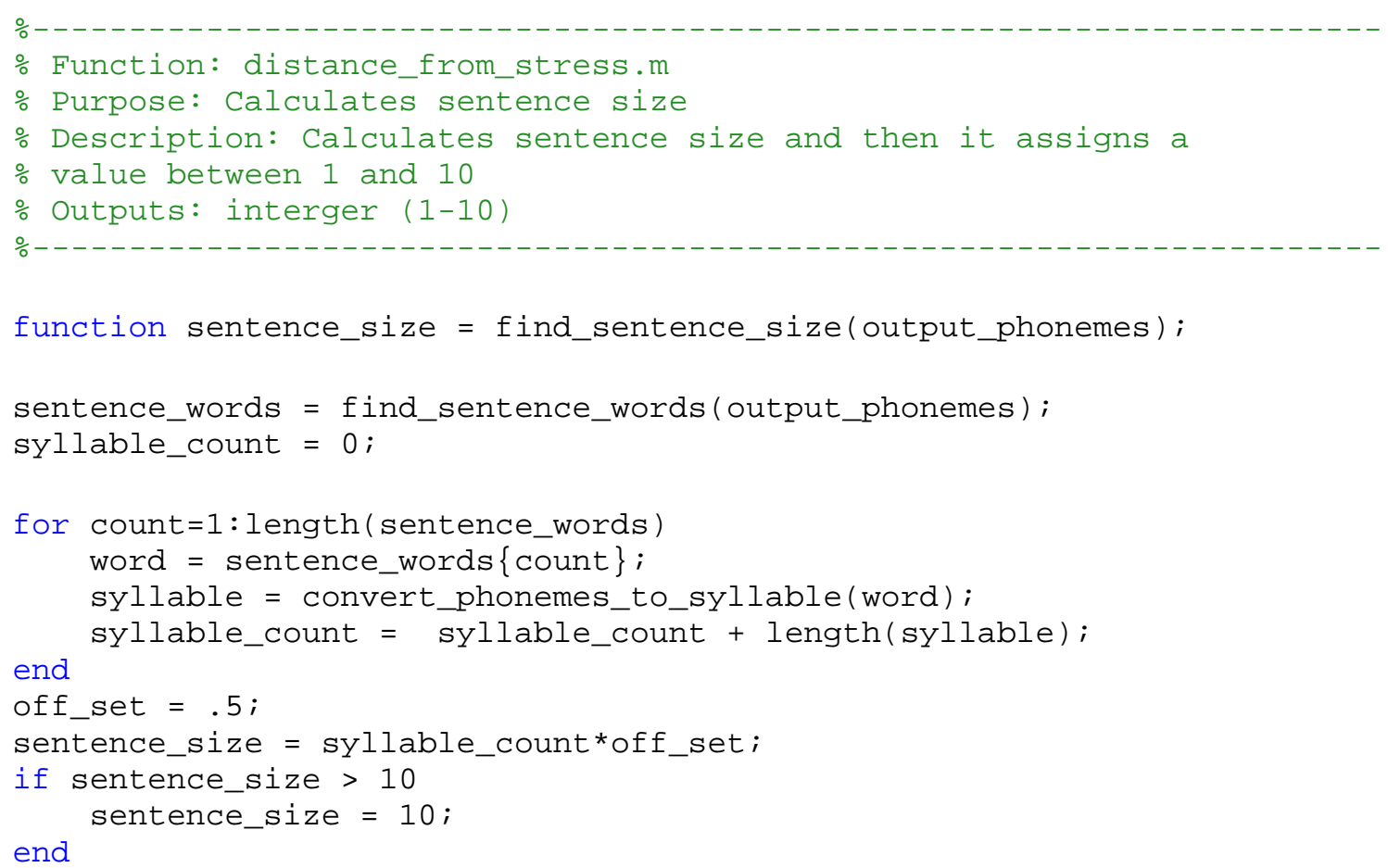




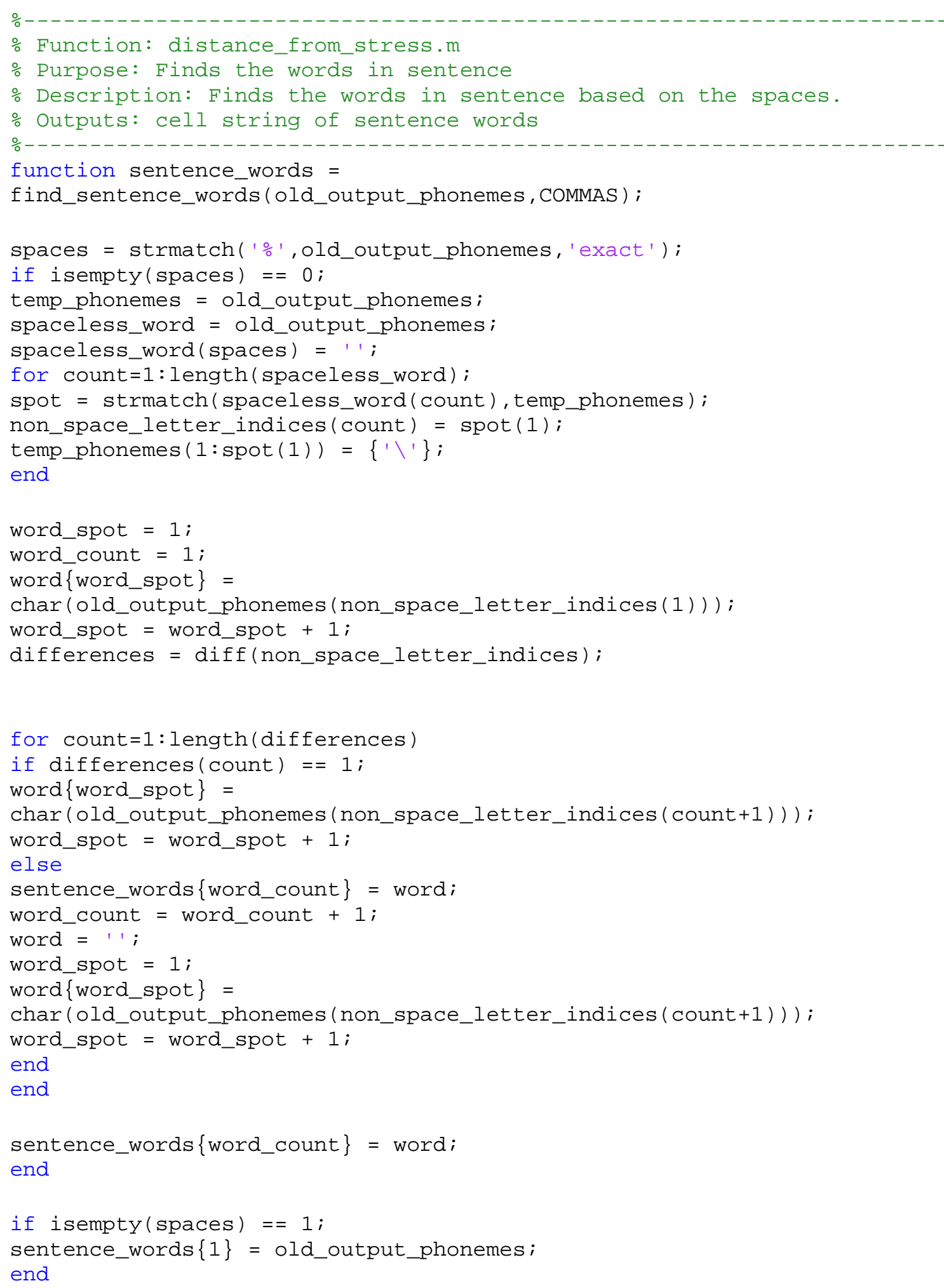

